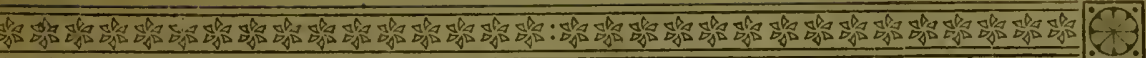

Peradeniya Manuals of Botany, Entomology, Agriculture, and Horticulture.

No. 2.

: A REVISED CATALOGUE

; $\quad \therefore \quad$ OF THE

\title{
FLOWERING PLANTS AND
} FERNS OF CEYLON,

NATIVE AND INTRODUCED.

By'J. C. WILLIS, M.A., Sc.D., S.D., Director, Royal Botanic Gardens, Peradeniya,

$\triangle N D$

M. WILLIS.

Price : in boards, Rs. $2 \cdot 50$; in cloth covers, Rs. 3 , or ts. $6 d$.

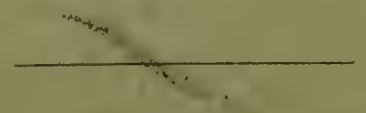

COLOMIBO :

11. C. COTTLE, GOVERNMENT PRIN'IER, CEYLON.

LONDON :

DULAU \& CO., 37, SOHO SQUARE.

1911. 


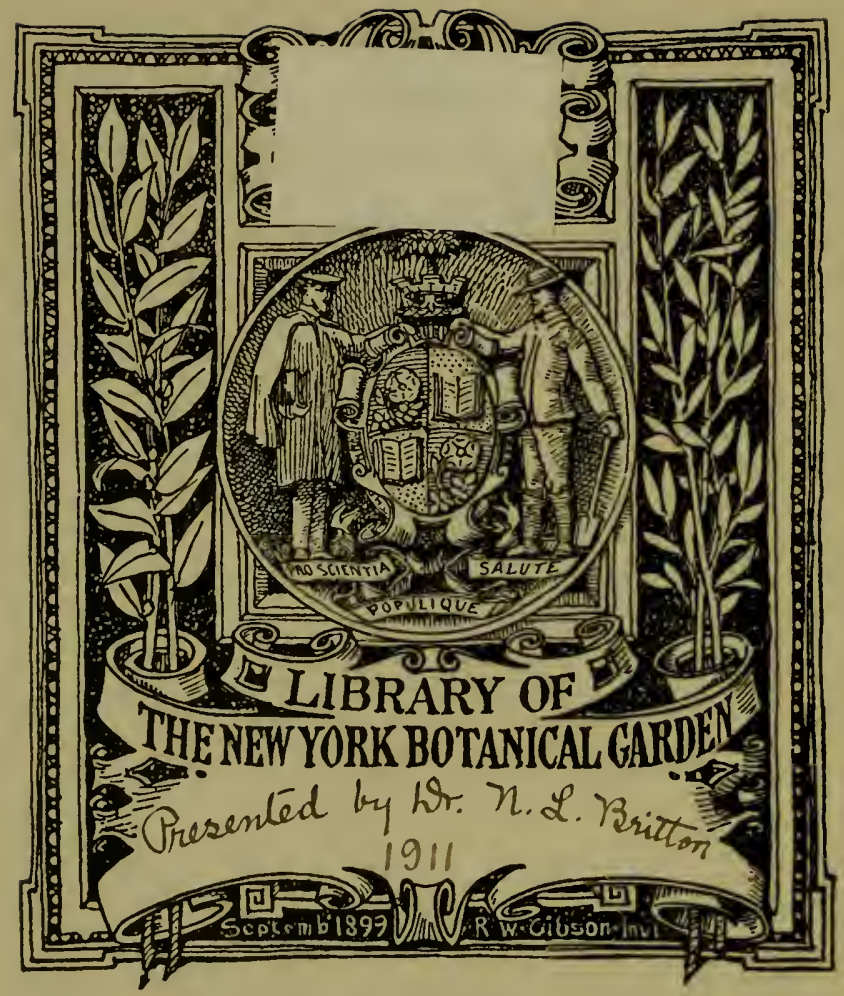




Peradeniya Manuals of Botany, Entomology, Agricuiture, and Horticulture.

No. 2.

A REVISED CATALOGUE

OF THE INDIGENOUS

FLOWERING PLANTS AND FERNS OF CEYLON.

BY

J. C. WILlis, M.A., Sc.D. (Cantab.), S.D. (Harv.), F.L.S. $\because \quad$ Director, Royal Botanic Gardens, Peradeniya.

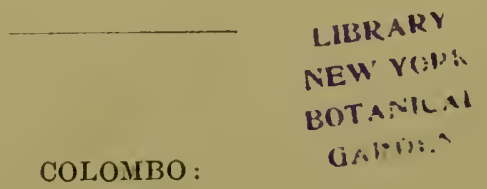

H. C. COTTLE, GOVERNIENT PRINTER, CEYLON. 

THE catalogue published by the late. Dr. Trimen in the 1 Journal of the Ceylon Branch of the Royal Asiatic Society for 1885 has long been out of print, and the want of a new one is often felt. I have therefore prepared the following list of the native plants of the Island to replace it.

After each name are given the Sinhalese (S.) and Tamil (T.) equivalents, if any, and sometimes an English name. After these is quoted the page in Trimen's " Flora of Ceylon" upon which the description of the species is to be found. If the species is figured in the "Plates" of the Flora, the number of the plate is also given.

Certain Orders have been revised since the publication of Trimen, and, where I have considered it necessary, I have used the revision, indicating the source in a footnote.

One great interest of the Ceylon flora lies in the enormous number of endemics which it contains. These are always printed in small capitals, with the exception of varieties. When a genus is thus printed, it means that the whole genus is endemic.

Names with an asterisk prefixed are apparently exotic species so fully naturalized in Ceylon that their foreign origin cannot be certainly sworn to. The many naturalized species that are without doubt introduced are not included.

\section{DICOTYLEDONS.}

POLYPETALE.

\section{Ranunculaceæ.}

1. Clematis, $\mathrm{L}$.

1. smilacifolia, Wall. Nara-wel, S.

2. Gouriana, Roxb.

2. Naravelia, DC.

3. zeylanica, DC. Nara.wel, S. 
3. Anemone, L.

4. rivularis, Ham.

4. Thalictrum, L.

5. Javanicum, Bl.

5. Ranunculus, L.

6. SAGITT жFolius, Hook. Bultercup.

7. Wallichianus, W. \& A. I.4

\section{Dilleniaceæ.}

6. Delima, L.

8. sarmentosa, L. Korasa-uel, S.

7. Tetracera, L.

9. lævis, Vahl. Et-korasa-wel, S.

§. Acrotrema, Jack.

10. UNIFLORUM, Hook. Bin-beru, S.

var. $\alpha$ petiolare, Thw.

var. $\beta$ rotundatum, Thw.

var. $\gamma$ bullatum, Hk. f. \& Th.

var. $\delta$ rugatum, Thw.

var. \& sylvaticum, Hk. f. \& Th.

var. $\zeta$ Walkeri, Trim.

var. $\eta$ membranaceum, Thw.

var. $\theta$ appendiculatum, Thw.

var. I dentatum, Thw.

11. INTERMEDIUM, Thw.

12. layceolatun, Hook.

13. GARDNERI, Thw.

14. ThwaITeSII, Hk. f. \& Th.

15. DISSECTUM, Thw.

16. LyRATUM, Thw.

9. Schuniacheria, Vahl.

17. CASTANemolia, Vahl. Kekiri-wara, S. I.10

18. AlNIFOLIA, Hk. f. \& Th.

var. $\beta$ dentata, Wight.

var. $\gamma$ subglabra, Thw.

19. ANGustifolia, Hk. f. \& Th. I.11, Pl. II.

10. Wormia, Rottb.

20. TRIQUETRA, Rottb. Diyapara, S. I.11, Pl. IJI.

11. Dillenia, L.

21. indica, L. Hondapara, Wampara, S.

22. Retusa, Thunb. Godapara, S. 


\section{$(3)$}

3. Magnoliaceæ.

12. Michelia, L

23. nilagirica, Zenk. Wal-sapu, S.

I.14

var. $\beta$ ovalifolia, Thw.

var. $\gamma$ Walkeri, Thw.

13. Kadsura, Kæmpf.

24. Wightiana, Arn.

I.16

\section{Anonaceæ.}

14. Uvaria, L.

25. SPHENOCARPA, Hk. f. \& Tl.

I. 18

26. macrophylla, Roxb.

I. 18

27. semecarpifolia, Hk. f. \& Th.

28. MACROPODA, Hk. f. \& Th.

29. Narum, Wall.

30. zeylanica, L. Palukan, Pulanga, S. Karuveppal, $\mathrm{T}$.

15. Cyathocalyx, Champion.

31. zeylanicus, Champ. Kekala, Ipetta, s.

16. Artabotrys, $\mathrm{Br}$.

32. odoratissimus, $\mathrm{Br}$.

33. zeylanicus, Hk. f. \& Th. Pelika-wel, Yakadawel, S.

17. Unona, L.

34. ELegans, Thw.

35. zEYLANICA, Hk. f. \& Th.

18. Polyalthia, Bl.

36. Iongifolia, B. \& Hk. f. Marc-iluppai, T.

37. coffeoides, B. \& Flk. f. Omura, s. Nedunurai, $\mathrm{T}$.

38. acuminata, Thw.

39. Korinti, B. \& Hk. f. Miwenna, S. Ulwinlai, 'T.

40. suberosa, B. \& Hk. f. Kalati, S. $\quad \mathbf{I} 25$

41. MOONII, Thw.

42. PERSICIFOLIA, B. \& Hk. l.

19. Anaxagorea, St. Hil.

43. luzonensis, Gray.

20. Xylopia, L.

44. Parvifolia, Hk. f. \& Th. Netawu, Aluketiya,

S. Chidavintai, T.

45. NiGRICANS, Hk. f. \& 'Th.

46. champIonII, Hk. f. \& Th, Dat-ketiya, S. I.28 
21. Goniothalamus, Bl.

47. 'Thwaitesii, Hk. f. \& Th. Kulu-kera, S. I.29

48. $¥$ ARDNERI, Hk. f. \& Th. T.30

49. HOOKERI, Thw.

50. WALKERI, Hk. f. \& Th. Kapuru, S. I.30

51. THOMSONI, Thw. I.31

52. Reticulatus, Thw. I.31

53. SALICINUS, Hk. f. \& Th. I.31

22. Mitrephora, Bl.

54. Heyneana, Thw.

23. Bocagea, St. Hil. ${ }^{1}$

55. THWAITESI, Hk. f. \& Th. I.33

56. OBLIQUA, Hk. f. \& Th.

57. CORIACEA, Hk. f. \& Th. Keku, S. I.34

24. Miliusa, Leschen.

58. indica, Leschen.

var. $\beta$ tomentosa, Thw.

59. zeYLANICA, Gardn.

25. Orophea, Bl.

60. zeylanica, Hk. f. \& Th.

26. Alphonsea, Hk. f. \& Th.

61. lutea, Hk. f. \& Th.

I.36

62. zeylanica, Hk. f. \& Th. $\quad$ I.36

63. SCLEROCARPA, Thw.

1.37

5. Menispermaceæ.

27. Tinospora, Miers.

64. malabarica, Miers. Bu-kinda, Wal-kinda, S. I.38 var. $\beta$ tomentosa, Miers.

*65. crispa, Miers. Titta-kinda, S.

66. cordifolia, Miers. Rasa-kinda, S. Chintil, T. I.39

28. Anamirta, Colebr.

67. paniculata, Colebr. Titta-wel, S.

29. Coscinium, Colebr.

68. fenestratum, Colebr. Weni-wel, S. False Calumba.

30. Tiliacora, Colebr.

69. racemosa, Colebr.

31. Limacia, Lour.

70. cuspidata, Hk. f. \& Th. Niri-wel, S. I.42, Pl. IV.

King (Anonacer of British India, Ann. R. B. G. Calcutta) places 55 in Sagerœa, 56 and 57 in Orophea. 


\section{$(5)$}

32. Cocculus, DC.

71. macrocarpus, W. \& A.

72. villosus, DC.

33. Pachygone, Miers.

73. ovata, Miers. Kaddukkodi, T.

34. Stephania, Lour.

74. hernandifolia, Walp. Lnunuketiya-wel, S.

35. Cissampelos, $\mathrm{L}$.

75. Pareira, L. Diyamitta, S.

36. Cyclea, Arn.

$$
\text { var. } \beta \text { subpeltata, Thw. }
$$

76. Burmanni, Miers. Kehi-pittan, Kesi-pissan, S. I.47

6. Berberideæ.

37. Rerberis, L.

77. aristata, DC. Barberry.

7. Nymphæaceæ.

38. Nymphæa, $L$.

78. Lotus, L. Olu, Et-olu, S. Waterlily, Egyptian

Lotus.

var. $\beta$ pubescens, Willd.

79. stellata, Willd. Manel, S. W'aterlily.

39. Nelumbium, Juss.

80. speciosum, Willd. Nelun, S. T'amarai, 'T. Sacred Bean, Lotus.

8. Cruciferæ.

40. Nasturtium, Br.

*81. indicum, DC.

41. Cardamine, $L$.

82. africana, $L$.

83. subumbellata, Hk. f.

9. Capparideæ.

42. Cleome, $L$.

84. monophylla, L.

85. tenella, L. f.

86. aspera, Kœen.

87. Chelidonii, L. f.

88. viscosa, L. WTal-aba, Ran-manissa, S. [.57

43. Gynandropsis, DC.

89. pentaphylla, DC. Wela, S. Tayirvalai, T. 
44. Mærua, Forsk.

90. arenaria, Hk. f. \& Th.

45. Cratæva, L.

91. Roxburghii, Br. Lunu-warana, S. Navala, Navilankai, 'T.

46. Cadaba, Forsk.

92. trifoliata, W. \& A. Maya'adikikruntu, T.

93. indica, Lam. Vili, T.

47. Capparis, L.

94. zeylanica, L. Kattotdi, Vennachchi, T.

95. divaricata, Lam.

96. Moonii, Wight.

97. Roxburghii, DC. Punaivirandi, $T$.

98. grandis, L. f. Mudkondai, T.

99. pedunculosa, Wall. Pichchuvilattai, Karunchurai, $\mathrm{T}$.

var. $\beta$ longispina, Hk. f. \& Th.

100. sepiaria, L. Karunchurai, T.

var. $\beta$ retusella, Thw.

101. Horibunda, Wight.

102. horrida, I. f. Welangiriya, S.

103. tenera, Dalz. var, zeylanica, Hk. f. \& Th.

\section{Violaceæ.}

48. Viola, L.

104. Patrinii, DC. Violet.

105. distans, Wall. Violet.

106. serpens, Wall. Violet.

49. Ionidium, Vent.

107. suffruticosum, Ging. Oritad-tamarai, T. I.67

108. RAMOSISSIMUM, Thw.

50. Alsodeia, Thouars.

109. zeylanica, Thw.

110. DECORA, Trim.

111. VIRGATA, Hk, f. \& Th.

\section{Bixaceæ.}

51. Scolopia, Schreb.

112. acuminata, Clos. Katu-kenda, $\mathrm{S}$.

113. CRASSIPES, Clos. 1.71

114. GARTNERI, Thw. Katu-kurundu, S. 1.71

52. Erythrospermum, Lam.

115. рнутоLAcCoInes, Gardn. 


\section{$(7)$}

53. Flacourtia, Comm.

116. Ramontchi, L'Herit. var. sapida, Roxb.

Uguressa, S. Katukali, Karumurukki, T.

117. sepiaria, Roxb. Mulanninchil, T.

54. Aberia, Hochst.

118. Gardnert, Clos. Kelambilla, S. I.74, PI. VII.

55. Trichadenia, Thw.

119. zeylanica, Thw. Tolol, Titta, S. I.75, Pl. VIII.

56. Hydnocarpus, Gærtn.

120. venenata, Gritn. Makulu, S. Makal, T. I.75

121. alpina, Wight. Gomma, S. Attuchankulai,

T.

122. octandra, Thw.

\section{Pittosporaceæ.}

57. Pittosporum, Banks.

123. tetraspermum, W. \& A.

1.77

124. zexlanictim, Wight. Ketiya, S.

\section{Polygalaceæ.}

58. Polygala, L.

125. arillata, Ham.

126. Javana, DC. Tilo-guru, S.

127. leptalea, DC.

128. GLAUCOIDES, L.

var. $\beta$ triflora, $\mathrm{L}$.

var. $\gamma$ hirsutula (Arn.), Trim.

129. chinensis, L.

130. rosmarinifolia, W. \& A.

131. sibirica, L. var. MACROLOPHOS, Hassk. I.82

132. telephioides, Willd.

59. Salomonia, Lour.

133. oblongifolia, DC.

134. Cordata, Arn.

60. Xanthophyllum, Roxb.

135. flavescens, Roxb. Palala, S.

14. Caryophyllaceæ.

61. Cerastium, I.

136. indicum, W. \& A. $\quad$ I.85

*137. vulgatum, L. var. glomeratum, Thuill. I.85

62. Stellaria, L.

138. DRYMARIOIDES, Thw. 
63. Drymaria, Willd.

139. cordata, Willd. Kukulu-pala, S. $\quad$ I.87

64. Polycarpon, L.

140. Loflingiæ, B. \& Hk. f.

65. Polycarpæa, Lam.

141. corymbosa, Lam.

I.88

\section{Portulacaceæ.}

66. Portulaca, L.

142. oleracea, L. Genda-kola, S. Pulikkirai, Pulichchankirai, T.

143. Wightiana, Wall. $\quad$ I.89

144. quadrifida, L. Hin-genda-kola, s. $\quad$ I.90

145. tuberosa, Roxb. Ura-genda, S. $\quad 1.90$

146. suffruticosa, Wight. $\quad$ I.90

16. Tamariscineæ.

67. Tamarix, L.

147. gallica, L. Kirai, T'ini, T.

17. Elatineæ.

68. Bergia, L.

148. ammannioides, Roxh.

149. verticillata, Willd.

18. Hypericaceæ.

69. Hypericum, L.

150. mysorense, Heyne. $\quad 1.93$

15l. japonicum, Thunb. $\quad$ I.93

19. Guttiferæ.

70. Garcinia, L.

152. Cambogia, Desrouss. Goraka, S. Korakkaipuli, T.

var. $\beta$ Papilla, Wight.

153. Morella, Desrouss. Krna-goraka, Gokatu,

Kokatiya, S. Gamboge.

154. ECHINOCARPA, Thw. Madol, S.

155. TIERPNOPHYLLA, 'Thw. Kokatiya, S. var. $\beta$ acuminata, Thw.

156. spicata, Hk. f. Ela-gokatu, Gonapana, S.

Kokottai, T. var. $\beta$ acutifolia, T. And. 
71. Calophyllum, L.

157. spectabile, Willd. Domba-kina, II apat-kina, S. I.99

158. BURManNI, Wight. Ciuru-kinu, Hin-kina,

S. Chirupunnai, T.

159. pulcherrimum, Wall.

160. Inophyllum, L. Domba, Tel-domba, S. Punnai, Dommakotlai, T.

161. tomentosum, Wight. Kina, S.

162. BRaCteatum, Thw. Wall-kina, S.

163. ThWAItesir, Planch. \& Tri.

164. TRAPEZIFOLIUM, Thw -

165. CUNEIFOLIUM, Thw.

166. CORDATO-OBLONGUM, Thw.

167. WaLKERr, Wight. Kina, S.

72. Kayea, Wall.

168. STYLOSA, Thw. Suwanda, S.

1.104

73. Mesua, L.

169. ferrea, L. $N a$, S. Naka, T'. Iron-wood. I.105 var. $\beta$ sclerophylla, Thw. var. $\gamma$ pulchella, Planch. \& Tri.

170. тhwaitesir, Planch. \& Tri. Diya-na, S. I.106 20. Ternstrœmiaceæ.

74. Ternstrœmia, L.

171. japonica, Thunb.

172. EMARginata, Choisy.

75. Adinandra, Jack.

173. LaSIOPETALa, Choisy. I.108, PI. IX.

76. Eurya, Thunb.

174. japonica, Thunb. Neya-dasse, S. I. 109 var. $\alpha$ Thunhergii, Thw. var. $\beta$ zeylanica, Wight. var. $\gamma$ Chinensis, Br.

175. acuminata, DC.

77. Gordonia, Ellis.

176. zEYLANICA, Wight. var. $\beta$ elliptica, Gardn.

177. SPECIOSA, Choisy.

\section{Dipterocarpaceæ.}

78. Dipterocarpus, Gærtı. f.

178. Hispidus, Thw. Bu-hora, S.

179. ZeYlanicus, Thw. Hora, S.

180. SCABRIDUS, Thw.

181. glannulostes, Thw. Dorame, S.

182. INSIGNIS, Thw. 
79. Shorea, Roxb.

18:3. oblonerfolia, Thw.

I.116

184. DYERII, Thw.

1.117

185. RETICULATA, Thw.

1.117

186. LISSOPHYLLA, Thw.

I.117

187. STIPULARIS, Thw.

1.118

80. Doona, Thw.

$$
\text { var. } \beta \text { minor, Thw. }
$$

188. zeYlaniCa, Thw. Dun, S.

189. AFFINIS, Thw.

190. Gardneri, Thw. Red Doon.

I.121

191. nervosa, Thw. Kotikan-beraliya, S. Red Doon.

192. TRAPEZIFOLIA, Thw. Yakahalu, S.

193. CongestiflorA, Thw. Tiniya, S. I.122, Pl. XI. 194. Cordifolia, Thw. Beraliya, S.

I.1 22

195. ovalifolia, Thw. Pini-beraliya, S.

I.123

196. OBLONGA, Thw.

$\mathrm{I} .123$

197. VENULOSA, Thw.

I.1.23

198. MaCrophylda, Thw. Honda-beraliya, S. I.124

81. Hopea, Roxb.

199. DISCOLOR, Thw.

1.125

200. JUCUNDA, Thw. Rat-beraliya, S.

1.125

var. $\beta$ modesta, $A$. DC.

201. CORDIFOLIA, Trim.

1.126

82. Sunaptea, Griff.

202. SCABRIUSCULA, Trim. Na-mendora, S. I.126,

Pl. XII.

203. ? DISTICHA, Trim.

83. Vatica, L.

204. Roxburghiana, B1. Mendora, S.

I.1.28

205. AFFINIS, Thw. Hal-mendora, S.

I.128

206. obscura, Trim. Tumpalai, T. 1.129, Pl. X[11.

84. Balanocarpus, Bedd.

207. ? zeylanicus, Trim. 1.130, PI. XIV.

85. Vateria, L.

208. acuminata, Hayne. Hal, S.

86. Stemonoporus, Thw.

209. WIGHTII, 'Thw. Hal-mendora, S.

1.132

210. GARDNERI, Thw.

1.133

211. acuminatus, Bedd.

1.133

212. LANCEOIATUS, Thw.

[.134

213. AFFINIS, Thw.

I.l34, PI. XY.

214. RIGIDUs, Thw.

I. 134

215. Canaliculatus, Thw. 
216. PETIOLARIS, Thw.

217. OBLONGIFOLIUS, Thw.

I.135

218. RETICUlatus, Thw.

I.135

219. NITIDUS, Thw.

I.136

var. $\beta$ lancifolius, Dyer.

I.136

220. NeRvosus, Thw.

I.136

221. (?) MOONח, Thw.

I.137

222. Lifistands, Trim. Mss.

V.383

223. (?) Revolutus, Trim. Mss.

V.384

87. MoNoporandra, Thw.

224. CORDIFOLIA, Thw.

I.137

225. ELEGANS, Thw.

I.138

21 bis. Ancistrocladaceæ.

88. Ancistrocladus, Wall.

226. vaHLI, Arn. Gona-wel, S.

I.139, PI. XVI.

22. Malvaceæ.

89. Sida, $\mathrm{L}$.

227. humilis, Cav. Bevila, S. Palam-padu, Palam-pasi, T.

I.141

228. mysorensis, W. \& A. Giriwadi-bevila, S. $\mathrm{I} .142$

229. spinosa, $\mathrm{L}$.

I. 142

230. acuta, Burm. Gas-bevila, S.

I.142

231. rhombifolia,L. Kotikan-bevila, S. Chittamaddi, $\mathrm{T}$. var. $\beta$ retusa, $\mathrm{L}$.

I.143

232. cordifolia, L. Wal-bevila, Hin-anoda, S.

Chevakanpudu, T.

I.143

90. Abutilon, Gærtn.

233. asiaticum, G. Don. Anoda, S.

234. muticum, G. Don.

I.144

235. indicum, G. Don. Anoda, S. Peruntutti, Vaddattutti, T.

I.145

236. graveolens, W. \& A. Vaddattutti, T.

I. 145

237. crispum, G. Don.

I. 145

I.146

91. Wissadula, Medik.

238. zeylanica, Medik. Kiri-kaju, S.

I. 146

92. Urena, $\mathrm{L}$.

239. lobata, L. Patta-epala, S.

240. sinuata, L. Hin-epala, S.

93. Pavonia, Cav.

241. glechomifolia, A. Rich.

242. zeylanica, Cav.

I. 148

243. odorata, Willd. 
94. Julostrlis, Thw.

244. ANGUSTIFOLLA, Thw:

95. Dicellostyles, Benth.

245. AXILLARIS, Benth.

96. Hibiscus, L.

246. surattensis, L. Hin-napiritta, S. $\quad \mathrm{I} .152$

247. furcatus, Roxb. Napiritta, S.

$\mathrm{I} .152$

248. collinus, Roxb. Parutti, T.

I.152

249. lunariifolius, Willd.

1.153

250. micranthus, L. f. Perumaddi, T.

I.153

251. vitifolius, L. Maha-epala, S. Vaddattutti. T. I.154

252. panduræformis, Burm.

I.154

253. Solandra, L'Herit.

I. 155

254. ficulneus, $\mathrm{L}$.

I.155

*255. Abeimoschus, L. Kapu-kinissa, S.

I.156

256. angulosus, Mast. Kapu-kinissa, S. I.156, Pl.XVII. var. $\alpha$ grandiflorus, Thw. var. $\beta$ purpureus, Thw.

257. tiliaceus, L. Beli-patta, S.

97. Thespesia, Corr.

258. Lampas, Dalz. \& Gibs.

259. populnea, Soland. ex Corr. Suriya, S.

1.158

Kavarachu, Puvarachu, T. Tulip Tree.

I.158

98. Bombax, L.

260. malabaricum, DC. Katu-imbul, S. Parutti, Kaddu-parutti, T. Cotton Tree.

99. Eriodendron, DC.

*261. anfractuosum, DC. Imbul, Pulun-imbul,

S. Silk-cotton Tree. .

1.161

100. Cullenia, Wight,

262. excelsa, Wight. Katu-boda, s.

\section{Sterculiaceæ.}

101. Sterculia, L.

263. fotida, L. Telambu, s.

264. urens, Roxb. Kavali, 'T'.

I.164

265. guttata, Roxb.

I.164

266. Balanghas, L. Nava, S.

I.165

267. colorata, Roxb. Malaiparutti, T.

I. 165

268. THWatTESII, Mast.

I.166

I.166

102. Heritiera, Ait.

269. littoralis, Dryand. Etunu, S. Chomuntiri, $\mathrm{T}$. 
103. Helicteres, L.

270. Isora, L. Timiyn, S. Vallampuri, 'I'. I.168

104. Ptcrospermum, Schreb.

271. suberifolium, Lanı. Welanga, S. Vimanku, T.

105. Pentapetes, L.

*272. phœnicea, L.

106. Melochia, L.

273. corchorifolia, L. Gal-kura, S.

107. Waltheria, L.

274. indica, L. Punnikki, T.

\section{Tiliaceæ.}

11)8. Pitgranthe, 'Thw.

275. verrucosA, 'Thw. Dik-wenna, S. Vidpani, '1.

109. Berrya, Roxb.

276. Anmonilla, Roxb. Hal-milla, S. Chavandalai, T. Trincomalee Wood.

110. Grewia, L.

277. columnaris, Sm. Kodi-taviddai, 'T.

*278. asiatica, L.

279. bracteata, Heyne.

280. DIPLOCARPA, Thw.

281. tiliæfolia, Vahl. Daminiya, S. Chadachchi, T.

283. orientalis, L. Wel-keliya, Wel-mediya, S. Taviddai, T.

284. polygama, Roxb. Bora-daminiya, S. Taviddai, T.

285. Microcos, L. Keliya, Kohu-kirilla, s. 286. populifolia, Vahl. Katu-peratti, Achchu,

$\mathrm{T}$.

I.178, Pl. XVIII.

111. Triumfetta, L.

287. tomentosa, Boj.

288. pilosa, Roth.

289. rhomboidea, Jacq. Épala, S.

290. GLABRA, Rottl.

I.179

291. neglecta, W. \& A.

112. Corchorus, L.

*292. capsularis, L. Jute.

293. olitorius, L. Jute.

I.18\%

294. urticæfolius, W. \& A. 
*295. fascicularis, Lam.

296. tridens, L.

I.183

297. acutangulus, Lam.

I.183

113. Elæocarpus, L.

298. serratus, L. Weralu, S. Wild Olive. I.184, PI. XX. 299. amœnus, Thw. Titta-weralu, S.

I.185

300. овоvatus, Arn.

I.186

301. MontanUs, Thw.

I.186

302. subvillosus, Arn. Gal-weralu, S. $\quad$ I.186

303. zeylanicus, Mast.

I.187

304. GLANDULIFER, Mast.

$\mathbf{5 . 1 8 7}$

\section{Linaceæ.}

114. Linum, L.

305. mysorense, Heyne.

I.188

115. Hugonia, L.

306. Mystax, L. Maha-getiya, Bugetiya, S. Motirakanni, T.

307. FERRUGINEA, W. \& A.

I.190

116. Erythroxylon, L. ${ }^{1}$

308. monogynum, Roxb. Devadaram, Chemmanatti, $\mathrm{T}$.

309. aCUMLi ATUM (Arn.) Walp. (LUCiddu, Moon).

Bata-kirilla, S. Chiru-chemmanatti, T.

310. lanceolatum, Hk. f.

311. овтUSIFOLIUM, Hk. f.

I.191

I.192

26. Malpighiaceæ.

117. Hiptage, Gærtn.

312. Madablota, Gærtn. Puwak-gediya-wel, S. I.193

313. parvifolia, W. \& A.

I 193

\section{Zygophyllaceæ.}

118. Tribulus, $\mathrm{L}$.

314. terrestris, L. Sembu-nerinchi, S. Chirunerinchi, T.

28. Geraniaceæ.

119. Geranium, L.

315. nepalense, Sweet.

120. Oxalis, L.

316. corniculata, L. Hin-embul-embiliya, S.

1 E. zeylanicum, n. sp., added by Schulz in Das Pflanzenreich. 
121. Biophytum, DC.

317. sensitivum, DC. (as-nidikumba, S. I.197

318. NERVIFOLIUM, Thw. I.198

319. NUDUM, Wight. I.198

320. intermedium, Wight. I.199, PI. XXI.

321. PROLIFERUM, Wight. I.199

122. Impatiens, L. Balsam.

322. acaulis, Arn.

I.201

323. oppositifolia, L.

I.202

324. cuspidata, W. \& A.

I.202

var. $\beta$ bipartita, Arn.

325. Balsamina, L. Kudalu-kola, S.

I.203 var. $\beta$ cornuta, Trim.

326. GLANDULIFERA, Arn.

I.203

327. MACROPHYLLA, Gardn.

I.204

328. Repens, Moon. Gal-demata, S. I.205

329. LEPTOPODA, Arn.

330. TRUNCATA, Thw.

I.205

331. flaccida, Arn. Kudalu-mal, S.

I.206

332. Henslowiana, Arn.

I.206

333. JANTHINA, Thw.

I. 207

334. HOOKERLANA, Ain.

I. 207

335. subcordata, Arn.

I.208

336. LEUCANTHA, Thw.

I. 208

337. LINEARIS, Arn.

I. 209

338. APPENDICULATA, Ain.

I.209

339. elongata, Am.

I. 209

340. CORNIGERA, Arn.

I. 210

341. ARNOTTII, Thw.

I. 210

342. WALKERI, Hook.

I.211

I.211

123. Hydrocera, B1.

343. angustifolia, B1. Diya-kudalu, Wal-kudalu,

S.

29. Rutaceæ.

124. Euodia, Forst.

344. Roxburghiana, Benth. Lunu-ankenda, S. I.214

125. Zanthoxylum, L.

345. - tetraspermum, W. \& A.

126. Toddalia, Juss.

346. aculeata, Pers. Kudu-miris, S. Kandai,T. I.215

127. Acronychia, Forst.

347. laurifolia, B1. Ankenda, S. 
128. Glycosmis, Correa.

348. pentaphylla, Corr. Dodan-pana, S. Kulapannai, T. var. $\beta$ longifolia, Oliv.

349. BILOCULARIS, Thw.

129. Nicromelum, BI.

350. pubescens, B1. Wal-karapincha, S. Kakaipalai, $\mathrm{T}$.

130. Murraya, L.

351. exotica, L. Etteriya, S.

352. GLENIEI, Thw.

353. Konigii, Spreng. Karapinche, S. Karivempu, T. Curry Leaf.

131. Clausena, Burm.

354. indica, Oliv. Migon-karapincha, s. Pannai, Purankainari, T.

355. Willdenovii, W. \& A. Weda-pana, S. var. $\beta$ pubescens, W. \& A.

132. Limonia, I.

356. alata, W. \& A. Tumpat-kurundu, S. $\quad$ I.223 357. crenulata, Roxb.

133. Luvunga, Hamilt.

358. eleutherandra, Dalz.

13t. Paramignya, Wight.

359. monophylla, Wight. Wellangiriya, S.

360. armata, Oliv.

I. 225

361. sp ?

$\mathrm{I} .225$

135. Atalantia, Correa.

362. monophylla, Correa. Perunkuruntu, T.

363. racemosa, W. \& A.

I. 226

364. zeylanica, Oliv. Yaki-naran, S. Pey-kuruntu, $\mathbf{T}$.

I. 226

var. $\beta$ rotundifolia, Oliv.

365. Missionis, Oliv. Pamburu, S. Kuruntu, I.227 Perum-kuruntu, T.

136. Feronia, Correa.

366. elephantum, Correa. Diwul, S. Vila, Vilatti, Mayaladikkuruntu, T. Elephant Apple, Wood-apple.

\section{Simarubaceæ.}

137. Ailantus, Desf.

367. malabarica, DC. Kumbalu, Wal-bilin, S. I.230 
138. Samadera, Gærtn.

368. indica, Gærtn. Samadara, S.

139. Suriana, $\mathrm{L}$.

369. maritima, $\mathrm{I}$.

31. Ochnaceæ.

140. Ochna, L.

370. squarrosa, L. Mal-kera, S. Chilanti, T. 1.23:3 var. $\beta$ cordata, Thw.

371. Wightiana, Wall. Bo-kera, S. Kalkarai, T.

I.233 var. $\beta$ Moonii, Trim.

372. RUFESCENS, Thw.

I.234

141. Gomphia, Schreb.

373. angustifolia, Vahl. Bo-kera, S.

1.235

32. Burseraceæ.

142. Balsamodendrum, Kunth.

374. caudatum, March. Kilivai, T.

375. Berryi, Arn. Mulkilivai, T.

143. Canarium, L.

376. BRUNNEUM, Bedd. Maha-bulu-mora, S. 1.238

377. zeylanicum, B1. Kekuna, S. Pakkili-

PI. XXIII. pal, $\mathbf{T}$.

I.239

144. Filicium, Thw. ${ }^{1}$

378. decipiens, Thw. Pehimbiya, S. Chittiraivempu, T.

33. Meliaceæ.

145. Munronia, Wight.

379. Рणмша, Wight. Bin-kohomba, S.

I.242

146. Melia, L.

380. dubia, Cav. Lunu-midella, S. Malaiverupu, $\mathrm{T}$.

147. Azadirachta, A. Juss.

381. indica, A. Juss. Kohomba, S. Vempu, T. Margosa.

148. Cipadessa, B1.

382. fruticosa, B1. Hal-bembiya, S.

149. Aglaia, Lour.

383. APIOCARPA, Hiern.

I.245

384. Roxburghiana, Miq. Kannakommm, T. 
150. Dysoxylum, B1.

385. binectariferum, Hk. f.

var. $\beta$ pyriforme, Trim.

151. Pseudocarapa, Hemsl.

386. Championi, Hemsl. Gona-pana, S.

$\mathrm{I} .248$

PI. XXIV.

152. Amoora, Roxb.

387. Rohituka, W. \& A. Hingul, S.

I.249

153. Walsura, Roxb.

388. GARDNERI, Thw.

389. Piscidia, Roxb. Kiri-kon, Molpetta, S.

Chadavakku, T. var. $\beta$ acuminata, Trim.

154. Carapa, Aubl.

390. moluccensis, Lam.

I.251

155. Chickrassia, A. Juss.

391. tabularis, A. Juss. Hulan-hik, S. Aglai, Kaloti, T. Chittagong Wood.

156. Chloroxylon, DC.

392. Swietenia, DC. Buruta, S. Mutirai, T. Satinwood.

I. 253

\section{Chailletiaceæ.}

157. Chailletia, DC.

393. sumatrana, Miq. Balu-nakuta, S. (?)

I.254

\section{Olacineæ.}

158. Ximenia, L.

394. americana, Willd. Chiru-illantai, T.

159. Olax, L.

395. scandens, Roxb. Kadalranchi, T. I. 256

396. Wightiana, Wall. $\quad$ I.256

397. ZeYLanioa, L. Mella, S. I.257

160. Strombosia, B1.

398. zeylanica, Gardn.

161. Opilia, Roxb.

399. amentacea, Roxb.

162. Cansjera, Juss.

400. Rheedii, Gmel. Eta-mura, S.

163. Lasianthera, Beauv.

401. apicalis, Thw. Urukanu, Uruhonda, S. 
164. Gomphandra, Wall.

402. axillaris, Wall.

403. coriacea, Wight.

165. Apodytes, E. Moyer.

404. Gardneriana, Miers.

I. 262

166. Mappia, Jacq.

405. ovata, Miers. Gandapana, S.

I.262

var. $\beta$ Championiana, Miors.

167. Pyrenacantha, Hook.

406. volubilis, Hook.

I.263

36. Ilicineæ.

168. Ilex, L.

407. Walkeri, Wight \& Gardn. I.264

408. denticulata, Wall. $\quad$ I.265

409. Wightiana, Wall. Andun-wenna, S. I.265

37. Celastraceæ.

169. Euonymus, L.

410. REVOLUTUS, Wight.

I.267

411. THWATTESII, LaWs.

I.267

412. WALKERII, Wight.

I.267

170. Glyptopetalum, Thw.

413. zeylanicum, Thw.

I.268

171. Microtropis, Wall.

414. WALLICHIANA, Wight.

I.269

415. ramiflora, Wight.

I. 269

172. Kokoona, Thw.

416. zeylanica, Thw. Kokun, Wana-potu, S. I.270

173. Plenrostylia, Wight.

417. Wightii, W. \& A. Piyari, Panaka, S. Chiru-piyari, $\mathbf{T}$.

174. Elæodendron, Jacq. f.

418. glaucum, Pers. Neralu, S. Piyari, Perunpiyari, $\mathrm{T}$. var. $\beta$ montanum, Thw.

175. Celastrus, $\mathrm{L}$.

419. paniculatus, Willd. Duhudu, S.

176. Gymnosporia, W. \& A.

420. FRUticosA, Hk. f.

421. cmarginata, Hk. f.

177. Kurrimia, Wall.

422. Zeylanica, Arn. Palen, Et-heraliya, Uruhonda, S, Konnai, T. 
178. Hippocratea, L.

423. obtusifolia, Roxb. Diya-kirindi-wel, S.

I. 275

424. Arnottiana, Wight.

I.275

425. indica, Willd.

179. Salacia, L.

426. prinoides, DC. Hin-himbutu-wel, S.

I. 276

427. reticulata, Wight. Himbutu-wel, Kottala-

himbutu, S.

var. $\beta$ diandra, Thw.

428. oblonga, Wall. Chundan, T.

38. Rhamnaceæ.

180. Ventilago, Gærtn.

429. maderaspatana, Gærtn. Yakkada-wel, S.

Vempadam, T.

I.279

181. Zizyphus, Juss.

430. Jujuba, Lam. Maha-debara, S. Ilantai, T. I.280

431. Enoplia, Mill. Hin-eraminiya, S. Peri-

lantai, Churai, T.

432. NAPECA, Willd. Yak-eraminiya, S.

I. 280

var. $\beta$ lucida, Moon.

433. xylopyra, Willd. Kakuru, S. , Nari-ilantai, T.

I.281

434. rugosa, Lam. Maha-eraminiya, S. Churai, $\mathbf{T}$.

I.282

I.282

182. Rhamnus, L.

435. aRnotTianus, Gardn.

436. Wightii, W. \& A.

I.283

I.283

183. Seutia, Comm.

437. indica, Brongn. Tuvadi, Tudari, T.

I. 284

184. Sageretia, Brongn.

438. costata, Miq.

I.284

185. Colubrina, Rich.

439. asiatica, Brongn. Tel-hiriya, S. Mayirmanikkam, T.

I.285

186. Gouania, L.

440. microcarpa, DC.

39. Ampelideæ.

187. Vitis, L.

441. tomentosa, Heyne.

I. 288

442. indica, L. To-wel, Rata-bulat-wel, S.

I. 288

443. quadrangularis, Wall. Hiressa, S. Pirandai, $\mathbf{T}$.

44t. aLyptoca RPA, Laws. 
445. LONCHIPHYLLA, Laws.

446. adnata, Wall.

447. Linnaei, Wall. Wal-niviti, S. Kuddumuntiri, $\mathbf{T}$.

448. pallida, W. \& A.

449. repanda, W. \& A. Wal-diyalabu, S.

450. ACUMinata, Trim.

I.292

I.292

451. Heyneana, Wall.

1.292

452. Rheedii, W. \& A.

I.293

I.293

453. GARDNERI, Laws.

I.294

454. carnosa, Wall. Wal-rat-diyalabu, S.

I.294

455. Reticulata, Laws.

I.295

456. pedata, Vahl. Mediya-wel, S.

I.295

457. tenuifolia, W. \& A.

I. 296

458. setosa, Wall. Anaittadichchai, T.

I.296

459. lanceolaria, Wall. var. $\beta$ glycosmoides, Planch.

188. Leea, L.

460. sambucina, Willd. Burulla, Gurulla, S.

I. 297

40. Sapindaceæ.

189. Cardiospermum, L.

461. Halicacabum, L. Penela-wel, S.

I. 299

462. Corindum, $\mathrm{L}$.

$\mathrm{I} .300$

190. Hemigyrosa, B1.

463. canescens, Thw.

I.301 var. $\beta$ trichocarpa, Thw.

191. Allophylus, $\mathrm{L}$.

464. zeylanicus, L. Wal-kobbe, S.

$\mathrm{I} .302$ var. $\beta$ acuminatus, Hiern.

var. $\gamma$ varians, Hiern.

465. HISPIDUS, Trim.

466. Cobbe, B1. Kobbe, Bu-kobbe, S. Amarai, T. I.303 var. $\beta$ villosus, Hiern.

192. Schleichera, Willd.

467. trijuga, Willd. Kon, S. Puvu, Kula, T.

Ceylon Oak.

193. Gleniea, Hk. $f$.

468. ZEYLANICA, Hk.f. Wal-mora, S. Kuma, 1.305, $\mathrm{T}$.

P1. XXV.

var. $\beta$ unijuga, Trim.

194. Sapindus, L.

469. laurifolius, Vahl. Kaha-penela, S.

1.306

470. emarginatus, Vahl. Pencla, S. Neykkordan, Panalai, 'T. 
471. bifoliatus, Hiern.

472. EREOTOS, Hiern.

473. Thwaitesit, Hiern.

195. Nephelium, L.

474. Longana, Camb. Mora, Rasa-mora, S.

Nurai, T. Longan.

1.309 var. $\beta$ pallida, Trim.

475. GardnerI, Thw. Nurai, T.

196. Pometia, f'olst.

476. eximia, Hk. f. Gal-mora, Bulu-mora, $N a$-imbul, S.

$\mathrm{I} .310$

197. Harpullia, Roxb.

477. imbricata, Thw. Na-imbul, Pundalu, S. $\quad 1.311$

198. Dodonæa, $\mathrm{L}$.

478. viscosa, L. Eta-werella, S. Virali, T.

199. Turpinia, Vent.

479. pomifera, DC. Eta-hivilla, Kankumbala, Kukul-man, $\mathrm{S}$. var. $\beta$ inontana, Thw.

\section{Sabiaceæ.}

200. Meliosma, B1.

480. Wightii, Planch.

481. Simplicifolia, Walp. Elbedda, S.

482. Arnottiana, Walp.

\section{Anacardiaceæ.}

201. Buchanania, Roxb.

483. angustifolia, Roxb.

202. Mangifera, $L$.

484. ZEYLANICA, Hk. f. Et-amba, Wal-amba, S. Kaddu-ma, T.

203. Odina, Roxb.

485. Wodier, Roxb. Hik, S. Odi, T.

204. Semecarpus, L. f.

486. MARginata, Thw.

487. subpeltata, Thw. Maha-badulla, S.

488. PUbescens, Thw.

var. $\beta$ Thwaitesii, Engl.

489. овоvata, Moon. Kalu-badulla, S.

490. MOONII, Thw.

491. Cortacea, Thw. Badulla, S. 


\section{( 23 )}

494. acuminata, Thw.

var. $\beta$ intermedia, Trim.

495. NIGROVIRIDIS, Thw.

496. obscura, Thw. Badulla, S.

I.324

497. PARVIFOLIA, Thw.

I.324

498. LæVIGATA, Thw.

205. Nothopegia, B1.

499. Colebrookiana, B1. Bala, S.

I.325

206. Campnosperma, Thw.

500. zeylanicum, Thw. Aridda, S.

I.326

207. Spondias, L.

501. mangifera, Willd. Embarella, S. Ampallai, T. Hog-plum.

43. Connaraceæ.

208. Rourea, Aubl.

502. santaloides, W. \& A. Kirindi-wel, S.

II.1

209. Connarus, L.

503. monocarpus, L. Radaliya, S. Chettupulukodi, $\mathrm{T}$.

II.2

504. CHAMPIONII, Thw. Wel-radaliya, S. II.3

210. Ellipanthus, Hk. f.

505. THWAITESII, Hk. $f$.

II.3

\section{Leguminosæ.}

211. Rothia, Pers.

506. trifoliata, Pers.

II.7

212. Heylandia, DC.

507. latebrosa, DC.

II. 8

213. Crotalaria, L.

508. biflora, L.

509. prostrata, Roxb.

510. ferruginea, Grah.

511. evolvuloides, Wight.

512. MULTIFLORA, Benth.

II.9

II.9

-II. 10

II. 10

513. rubiginosa, Willd.

II.11

514. mysorensis, Roth.

II.11

II.12

*515. triquetra Dalz.

II. 12

516. albida, Heyne.

II. 12

517. nana, Burm.

II. 13

var. $\beta$ umbellata (Wight), Trim.

518. linifolia, L. f.

II.13

519. tecta, Heyne.

II.14

520. calycina, Schrank.

II.14 
521. retusa, L. Kaha-andana-hiriya, S. Kilukiluppai, T. var. $\beta$ maritima, Trim.

522. verrucosa, L. Nil-andana-hiriya, S. Kilukiluppai, T.

523. WALKerI, Arn.

II.15

524. juncea, L. Hana, S. Hemp, Sunn-hemp. II.16

525. lunulata, Heyne.

526. medicaginea, Lam.

var. $\beta$ luxurians, Baker.

527. striata, DC. var. $\beta$ acutifolia, Trim.

528. clavata, W. \& A.

II. 17

II.18

529. laburnifolia, L. Yak-beriya, S.

530. quinquefolia, L.

214. Parochetus, Ham.

531. communis, Ham.

215. Indigofera, L.

532. echinata, Willd.

533. linifolia, Retz.

II. 22

534. enneaphylla, L. Cheppunerinchi, T.

II. 22

535. aspalathoides, Vahl. Rat-kohomba, S.

Chivanarvempu, T.

536. glabra, L.

537. tenuifolia, Rott].

II.23

II. 23

538. viscosa, Lam.

II. 24

539. trifoliata, L.

II. 24

540. trita, L. f. Wal-awari, S.

II.24

541. subulata, Poir.

II. 25

542. paucifolia, Del. Nanti, Kut/ukarasamatti, T.

II. 25

543. hirsuta, L.

II.25

II. 26

*544. tinctoria, L. Nil-awari, S. Indigo.

545. constricta, Trim.

-546. Wightii, Grah.

II.26

547. galegoides, DC.

II. 27

II.27

II. 28

216. Psoralea, L.

548. corylifolia, L. Bodi, S.

II.28

217. Mundulea, DC.

549. suberosa, Benth.

II.29

218. Tephrosia, Pers.

550. spinosa, Pers.

II.30

551. senticosa, Pers.

II.30

552. tinctoria, Pers. Alu-pila, S.

II.31

var. $\beta$ pulcherrima, Baker. 


\section{$(25)$}

553. purpurea, Pers. Pila, S. Kolinchi, Kavilai, T. var. $\beta$ pumila, Baker.

554. maxima, Pers.

II.32, $1 \mathrm{Pl} . \mathrm{XXVII}$.

555. Hookeriana, W. \& A.

II.32

556. villosa, Pers. Bu-pila, S.

II.33

219. Sesbania, Pers.

*557. ægyptiaca, Pers. Chittakatti, T.

II.34

558. aculeata, Pers.

II.34

var. sericea, Benth.

220. Zornia, Gmel.

559. diphylla, Pers.

var. $\beta$ conjugata, Trim.

var. $\gamma$ Walkeri, Baker.

II.35

221. Stylosanthes, Sw.

560. mucronata, Willd. Wal-nanu, S.

II.36

222. Smithia, Aiton.

561. sensitiva, Ait.

II.37

562. conferta, Sm.

II.37

563. blanda, Wall.

II.37

223. Eschynomene, L.

564. indica, L. Diya-siyambala, S.

565. aspera, L. Maha-diya-siyambala, S. Attuneddi, T. Shola-pith.

II.38

II.39

224. Ormocarpum, Beauv. 566. sennoides, DC.

II.39

225. Eleiotis, DC.

567. sororia, DC.

II.40

226. Pycnospora, R. Br.

568. hedysaroides, $\mathrm{Br}$.

II.41

227. Pseudarthria, W. \& A.

569. viscida, W. \& A. Gas-gonika, S.

II.41

228. Uraria, Desv.

570. picta, Desv.

II.42

571. hamosa, Wall.

II.43

229. Alyssicarpus, Neck.

572. monilifer, DC.

II. 43

573. vaginalis, DC. Aswenna, S.

II.44

574. bupleurifolius, DC. Kutiraivali, T.

II.44

575. longifolius, W. \& A.

II. 45

576. rugosus, DC.

II.45

$$
\text { var. } \beta \text { Heyneanus, Baker. }
$$


230. Desmodium, Desv.

577. umbellatum, DC.

578. Cephalotes, Wall.

II. 47

579. pulchellum, Benth. Hampilla, S.

II. 48

580. biarticulatum, Benth.

II. 48

581. laburnifolium, DC.

II. 48

582. triquetrum, DC. Baloliya, S.

II. 49

583. ormocarpoides, DC.

II.49

584. Gardneri, Benth.

II. 50

585. Scalpe, DC.

586. gangeticum, DC.

II.50

587. latifolium, DC.

II.51

588. Thwaitesir, Baker.

II.51

589. Wightii, Grah.

II.51

590. rufescens, DC.

II. .52

591. heterocarpum, DC. Et-undupiyali, S.

II.52 var. $\beta$ trichocaulon, Baker.

592. JUCUNDUM, Thw.

II.54, Pl. XXVIII.

593. triflorum, DC. Hin-undupiyali, S.

II. 54

594. heterophyllum, DC. Maha-undupiyali, S. II.55

595. parvifolium, DC.

596. gyrans, DC. Chanchala, S. Telegraph

Plant.

II.56

*597. gyroides, DC.

II. 56

231. Abrus, L.

598. precatorius, L. Olinda-wel, S. Kuntumani, T. Crab's Eyes, Indian Liquorice. 599. pulchellus, Wall.

232. Shuteria, W. \& A.

600 . vestita, W. \& A.

233. Dumasia, DC.

601. villosa, DC. var. leiocarpa, Benth.

II. $5 \mathrm{~S}$

234. Glycine, L.

602. javanica, L. Goradiya, S.

235. Teramnus, Sw.

603. labialis, Spreng.

236. Mucuna, Adans.

604. monosperma, DC.

605. atropurpurea, DC. Punnakalichi, Phandatullai, T. 606. gigantea, DC.

607. pruriens, DC. Achariya-pala, S. Cowitch. 11.62 var. $\beta$ biflora, Trim. 
237. Erythrina, L.

608. indica, Lam. Erabadu, S. Mullu-

murukku, T.

609. ovalifolia, Roxb. Yak-erabadu, S.

238. Ștrongylodon, Vog.

610. ruber, Vogel.

239. Galactia, P. Br.

611. tenuiflora, W. \& A.

II. 65

240. Butea, Roxb.

612. trondosa, Kon. Gas-kela, S. Parasu, T. Bengal Kino.

II.66

241. Canavailia, DC.

613. ensiformis, DC. Wal-awara, S. Koliavarai, T. Sabre Bean.

II.67 var. $\beta$ virosa, Baker.

614. obtusifolia, DC. Mudu-awara, S.

II. 68

242. Dioclea, H. B. K.

615. reflexa, Hk. f.

[I.68

243. Phaseolus, L.

616. adenanthus, Meyer. Wal-me, S. II.70

617. Grahamianus, W. \& A.

II.70

*618. semierectus, L.

II. 70

619. trilobus, Ait. Bin-me, S. Narippayaru, T. II.71

620. aconitifolius, Jacq.

II.71

621. Max, L. Mun, Mun-eta, Bu-me, S.

Chiruppayaru, T. Green Gram.

var. $\beta$ radiatus, Baker. Ulundu, S.

Uluntu, T. Black Gram.

622. trinervius, Heyne.

623. calcaratus, Roxb.

II.72

II.72

II.73

244. Vigna, Savi.

624. luteola, Benth.

625. vexillata, Benth.

$[1.73$

11.74

245. Clitoria, L.

626. ternatea, L. Nil-katarodu, Kataroduwel, S. Kuruttappu, T.

246. Dolichos, L.

627. Lablab, L. Tatta-payaru, Minni, T. $\quad$ II.76

628. uniflorus, Lam. var. glaber, Thw.

II. 76

629. ciliatus, Kloin.

II. 77

630. falcatus, Kloin. Wal-dambala, S. 
247. Atylosia, W. \& A.

631. Candollei, W. \& A. Et-lora, S. II.78

632. albicans, Benth.

II. .78

633. rugosa, W. \& A. Wal-kollu, S.

11.79

634. scarabæoides, Benth. Wal-kollu, s'.

II.79

248. Dunbaria, W. \& A.

635. ferruginea, W. \& A

II. 80

636. Heynei, W. \& A.

II.80

249. Eriosema, DC.

637. chinense, Vogel.

II.81

250. Rhynchosia, Lour.

638. rufescens, DC.

II. 82

639. nummularia, DC.

II. 82

610. suaveolens, DC.

II. 83

641. cana, DC.

II. 83

642. cyanosperma, Benth.

II. 84

643. minima, DC.

II. 84

644. viscosa, DC.

II. 85

645. acutissima, Thw.

II. 85

646. densiflora, DC.

II. 85

251. Flemingia, Roxb.

II. 87

647. strobilifera, Br. Hampinna, S.

II. 86

648. lineata, Roxb.

II.87

649. congesta, Roxb.

II.87

var. $\beta$ semialata, Baker.

252. Dalbergia, L. f.

650. psoudo-Sissoo, Miq. (Championii, Thw.) $)^{1}$

Bambara-wel, S.

II. 88

651. lanceolaria, L. f. Veluruvai, T.

II. 88

652. torta, Grah. (monosperma, Dalz.)

II. 89

253. Pterocarpus, L.

653. Marsupium, Roxb. Gammalu, S. Venkai, T. II.90

254. Pongamia, Vent.

654. glabra, Vent. Magul-karanda, S. Punku, T. II.91

255. Derris, Lour.

655. scandens, Benth. Kala-wel, S. 'T'ekil, T. II.91

656. PARVIFLORA, Benth.

II. 92

657. uliginosa, Benth. Kala-wel, S.

II.92

658. Paniculate, Benth.

II.93

659. oblonga, Benth.

XIX.

660. sinuata, Benth.

II.94

1 Prain, Monugraph of D. Ann. R. P.C. Calcutta, X., p. 60. 


\section{$(29)$}

256. Sophora, L.

661. tomentosa, L. Mudu-murunga, S.

662. VIOLACEA, Thw.

663. ZEYLANICA, Trim.

25̃. Pericopsis, Thw.

664. MOONIANA, Thw. Nedun, S. II.97, Pl. XXXI.

258. Cæsalpinia, L.

665. Bonduc, Roxb. Kumburu-vel, S. Punaikkalaichchi, $\mathrm{T}$.

666. Nuga, Ait. Diya-wawul-etiya, S.

667. sepiaria, Roxb.

II.98

II. .99 var. $\beta$ auricoma, Trim.

668. digyna, Rottl.

259. Peltophorum, Vogel.

669. ferrugineum, Benth. Iya-vakai, 'T'.

260. Mezoneurum, Desf.

670. enneaphyllum, W. \& A. Goda-wawuletiya, S.

261. Cassia, L.

671. Fistula, L. Ehela, S. Tirukkontai, Kavani, $\mathrm{T}$.

672. marginata, Roxb. Ratu-wa, S. Vakkai, T. II.104 673. occidentalis, L. Peni-tora, S. Ponnantalarai, $\mathrm{T}$.

674. Sophera, L. Uru-tora, S. Takarai, T. II.105 675. Tora, L. Peti-tora, S. Vaddu-takarai, T. II.106 676. auriculata, L. Rana-wara, S. Avarai, T.

Matara Tea. II.106, P]. XXXII.

677. obtusa, Roxb. Nilavakai, T.

II. 107

678. siamea, Lam. Wa, Aramana.S. Vakai, T. II.108

679. timoriensis, DC.

II. 108

680. Absus, L. Bu-tora, S.

II. 109

681. Kleinii, W. \& A. Bin-siyambaln. S.

II. 110

var. $\beta$ pilosa. Thw.

682. mimosoides, L. Bin-siyambala, S.

II. 110

var. $\beta$ auricoma, Grah.

var. $\gamma$ Wallichiana, Baker.

262. Cynometra, L.

683. ramiflora, L. Gal-mendora, S. Attukaddupuli, T.

var. $\beta$ heterophylla, Thw.

263. Dialium, L.

684. ovoIDeum, Thw. Gal-siyambala, S. Kaddupuli, T. Velvet Tamarind. 
264. Crudia, Schreb.

685. ZEYLANICA, Benth.

265. Saraca, L. .

686. indica, L. Diya-ratmal, Diya-ratambala, S. II.114 266. Humboldtia, Vahl.

687. laurifolia, Vahl. Gal-karanda, S.

II.115

267. Bauhinia, L.

688. tomentosa, L. Petan, Kaha-petan, S.

Tiruvatti, T.

II. 116

689. racemosa, Lam. Mayila, S. Atti, T.

*690. anguina, Roxb.

II. 116

II.117

268. Neptunia, Lour.

691. oleracea, Lour. Diya-nidi-kumba, S.

II.118

269. Entada, Adans.

692. scandens, Benth. Pus-wel, S.

270. Adenanthera, L.

693. pavonina, L. Madatiya, S. Anaikuntu. mani, $\mathrm{T}$.

694. bicolor, Moon. Mas-mora, S. II.120, Pl. XXXIV.

271. Dichrostachys, W. \& A.

695. cinerea, W. \& A. Andara, S. Vidattal, T. II.121

272. Acacia, Willd.

*696. arabica, Willd. Karuvel, T.

II. 122

697. planifrons, W. \& A. Odai, Udai, T.

Umbrella Tree.

1I.123, Pl. XXXV.

698. eburnea, Willd. Udai-vel, Kaludai, T. Cockspur Thorn.

II.124

699. tomentosa, Willd. Anaimulli, T. Elephant Thorn, Jungle Nail.

II. 124

700. leucophlœa, Willd. Maha-andara, Katuandara, S. Velvel, T.

II.125

701. Sundra, DC. Rat-kihiri, S. Kodalimurunkai, T.

II. 125

702. ferruginea, DC.

703. cæsia, Willd. Hinguru, S.

II.126

704. pennata, Willd. Goda-hinguru, S.

II.127

II. 127

273. Albizzia, Durazz.

705. Lebbek, Benth. Mara, S. Kona, Vakai, T. II.128 706. odoratissima, Benth. Suriya-mara, Huriyi,

S. Ponnaimurankai, T.

II. 129

707. stipulata, Boiv. Kabal-mara, Hulanmara, $\mathrm{S}$.

708. amara, Boiv. Uyil, T. 
274. Pithecolobium, Mart.

709. aeminatum, Benth.

710. umbellatum, Benth. Iyamalai, Ichavalai, $\mathrm{T}$.

II.132

711. bigeminum, Benth. Kalatiya, S.

II. 132

712. subcoriaceum, Thw. Miminimara, S.

II. 133

\section{Rosaceæ.}

275. Pygeum, Gærtn.

713. Wightianum, Bl.

var. $\beta$ parvifolium. Thw.

714. zFYLANICUM, Gærtn. Golu-mora, Kankumbalketiya. S.

II. 135

276. Rubus, L.

715. glomeratus, $\mathrm{Bl}$.

716. moluccanus, L. Wel-bute, $\mathrm{S}$. var. $\beta$ Fairholmianus, Gardn. var. $\gamma$ macrocarpus, Gardn. Blackberry.

717. ellipticus, Sm.

718. lasiocarpus, Sm. var. $\beta$ subglaber, Thw.

277. Potentilla, L.

719. Mooniana, Wight.

720. Kleiniana, W. \& A.

278. Alchemilla, L.

721. indica, Gardn. var. $\beta$ sibthorpioides, Hk. $f$.

279. Poterium, L.

722. INDICUM, Gardn.

280. Agrimonia, L.

723. zeylanica, Moon.

281. Photinia, Lindl.

724. Notoniana, W. \& A.

II. 142

46. Saxifragaceæ.

282. Vahlia, Thunb.

725. oldenlandioides, Roxb.

II. 143

\section{Crassulaceæ.}

283. Kalanchoe, Adans.

726. floribunda, W. \& A. val', glabri, Clarke. II.144

727. laciniata, DC. 
48. Droseraceæ.

284. Drosera, L.

728. Burmanni, Vahl. Wata-essa, S. Sun-dex. II.145

729. indica, L. Kandul-essa, S. Sun-dew. II.146

730. peltata, Sm. Sun-dew. II.146

49. Haloragiaceæ.

285. Serpicula, L. ${ }^{1}$

731. ZEYLANICA, Ain.

II.147

var. $\beta$ minor, Clarke.

732. hirsuta, W. \& A.

II. 148

286. Myriophyllum, L.

733. indicum, Willd.

287. Callitriche, $\mathrm{L}$.

734. stagnalis, Scop.

50. Rhizophoraceæ.

288. Rhizophora, L.

735. mucronata, Jam. Kadol, S. Kandal, T. Mangrove.

II.151

736. Candelaria, DC. Kadol, S. Kandal, T. Mangrove.

II.151

289. Ceriops, Arn.

737. Candolleana, Arn. Chirukandal, 'I'.

II.152

738. Roxburghiana, Arn.

II.153

290. Bruguiera, Lam.

739. gymnorhiza, Lam. Mangrove.

II. 153

740. caryophylloides, $\mathrm{Bl}$.

II. 154

291. Carallia, Roxb.

741. integerrima, DC. Dawata, S.

742. CALYCINA, Benth. Ubberiya, S. II.155,

II.155

Pl. XXXV1.

292. Weihea, Spreng.

743. zeylanica, Baill. Pana, S. Kannu, T. II.156

293. Anisophyllea, $\mathrm{Br}$.

744. zEYlaNiCA, Benth. Weli-penna, Welipiyana, S.

II. 157

51. Combretaceæ.

294. Terminalia, L.

745. belerica, Roxb. Bulu, S. Tanti, T.

Myrobalans.

II.159

1 (1. Schindler in Das PHanzenreich. 
746. chebula, Retz. Aralu, S. Kadukkai, T. Myrobalans, Gall-nut, Ink-nut.

747. PARVIFLORA, Thw. Hanpalanda, S.

II.159

748.

295. Anogeissus, Wall.

749. latifolia, Wall. Dawu, S. Vekkali, 'I. II.162

296. Lumnitzera, Willd.

750. racemosa, Willd. Beriya, S.

II. 162

751. coccinea, W. \& A.

V. 385

297. Combretum, L.

752. acuminatum, Roxb.

753. ovalifolium, Roxb. Kaduru-ketiya-wel, S. II.163

754. extensum, Roxb.

298. Gyrocarpus, Jacq.

755. Jacquini, Roxb. Hima, S. Thinakku, T. II.165

52. Myrtaceæ.

299. Rhodomyrtus, DC.

756. tomentosa, Wight. Wild Guava. II.166

300. Eugenia, L.

757. aquea, Burm. Wal-jambu, S.

758. grandis, Wight.

II.169

II. 170

759. hemispherica, Wight.

II. 170

760. CYLINDRICA, Wight.

II.171

761. spicata, Lam. Maran, Maranda, S.

Marungi, T.

II.171

762. lanceolata, Lam.

763. Ferausoni, Trim. var. $\beta$ minor, Trin.

II.172

II.172, PI. XXXVIII.

764. lissophylla, Duth. Maha-kuretiya, S.

765. subavenis, Duth.

766. Gardneri, Duth. Dambu, S. Nir-naval, 'I'. II.174

767. corymbosa, Lam. Dan, Hin-dan, S. II.174

768. micrantha, Duth.

II. 175

769. revoluta, Wight.

II.175

770. SyLVestrIs, Wight. Alu-bo, S.

771. ASSIMILIS, Duth.

II. 175

772. CORDIFOLIA, Wight.

II.176

773. NeEsiana, Wight. Panu-kera, s.

II. 176

774. CYCLOPHYLLA, Thw.

II.177

775. ROTUNDIFOLIA, Wight.

II. 177

776. SClerophylla, Duth.

II. 177

777. oligantha, Duth.

II. 178

778. olivifolia, Duth.

II.178

II.178 
779. operculata, Roxb. Buta-damba, Kobomal, S.

780. Jambolana, Lam. Maha-dan, Madan, S.

Naval, Peru-naval, T. var. $\beta$ microcarpa, Thw.

781. lucida, Lam.

782. H eCKeltana, T'rim.

783. TERPNOPHYLLA, Thw.

II.179

784. XANTHOCARPA, Thw.

785. bracteata, Roxb. Tembiliya, S. Kaya, Venkalikaya, $\mathrm{T}$.

786. RUFO-FULVA, Thw.

787. PHILLYR ÆOIDES, Trim.

788. FLOCCIFERA, Thw.

II.180, PI. XXXVII.

789. RIVULORUM, Thw.

II. 181

II. 181

II. 182

790. FULVA, Thw.

791. INSIGNIS, Thw.

II. 182

792. DECORA, Thw.

II. 183

793. ROTUNDATA, Trim.

794. MAB EOIDES, Wight.

795. APRICA, Trim.

796. AMOENA, Thw.

II.183

797. PEDUNCULATA, Trim.

II.183

II.184

798. Mooniana, Wight. Pinibaru, S.

II. 184

799. Thwattesir, Duth.

II. 185

II. 185

II. 185

II.186

II. 186

II. 186

II. 187

II. 187

II. 188

301. Barringtonia, Forst.

800. speciosa, Forst. Mudilla, s.

801. racemosa, $\mathrm{Bl}$. Diya-midella, $\mathrm{S}$.

II. 189

802. zeylanica, Gardn. Goda-midella, S.

II. 189

803. acutangula, Gærtn. Ela-midella, S.

II.190

Alampu, T.

II.191

302. Careya, Roxb.

804. arborea, Roxb. Kahuta, S. Kuchaddai,

T. Patana Dak.

II.191

53. Melastomaceæ.

303. Osbeckia, L.

805. erythrocephala, Naud. Bowitiya, S.

II. 194

806. RHEEDI, Gardn.

II.194

807. zeylanica, L. f.

II. 195

808. aspera, Bl. Bowitiya, S.

II.195

var. $\beta$ minor, Trians.

var. $\gamma$ Ileinii, Arn.

var. $\delta$ Wightiana, Benth.

809. WALKERI, Arn.

var. 3 Beckettii, Thw. 


\section{$(35)$}

810. Buxifolia, Arn.

var. 3 minor, Thw.

811. RUBICUNDA, Arn.

812. MOONII, Thw.

II.198

813. octandra. DC.

II.198

304. Melastoma, L.

814. Malabathricum, I. Maha-bowitiya, S.

II.199

305. Kendrickia, Hk. f.

815. Walkeri, Hk. f.

II.200

306. Sonerila, Roxb.

816. ZeYLANICA, W. \& A.

var. $\beta$ pumila, Clarke.

II.202

var. $\gamma$ cordifolia, Thw.

var. $\delta$ affinis, Trim.

var. $\varepsilon$ rostrata, Clarke.

817. RHOMBIFOLIA, Thw.

II. 203

var. $\beta$ angustata, Thw.

818. Brunonis, W. \& A.

II. 203

819. Arnottiana, Thw.

II. 204

var. $\beta$ tomentella, Trim.

820. Wightiana, Arn.

var. $\beta$ Hookeriana, Trim.

821. HIRSUTULA, Ain.

822. GARDNERI, Thw. var. $\beta$ firma, Thw.

823. RoвUSta, Arn. var. $\beta$ glabricaulis, 'Thw.

var. $\gamma$ Harveyi, Trim.

824. LaNCEOlata, Thw.

JI.20t

II.205, Pl. XXXIX.

II. 205

II.206

825. PILOSULA, Thw.

II. 206

II. 207

826. linearis, Hk. f.

II. 20

827. pedunculosa, Thw.

11.208

307. Medinilla, Gaud.

828. Fuchsiordes, Gardn.

829. MACULATA, Gardn.

I1.20s

II.209, Pl. XL.

var. $\beta$ cuneata, Thw.

308. Memecylon, L.

830. ARNOTTLANUM, Wight.

LI.211

831. GARDNERI, Thw.

[I. 211

832. HOOKERI, Thw.

var. $\beta$ exalatum, Trim. Kevitiya-kera, S.

833. Wightii, Thw.

II. 212

var. $\beta$ cylindricum, Trim.

834. PARVIFOLIOM, Thw.

II. 213

835. VARLANS, Thw.

II. 213

var. $\beta$ rotundatum, 'Th $w$. 
836. elegantulum, Thw.

II. 214

837. ELLIPTICUM, Thw.

II. 214

838. MACROPHYLLUM, ThW.

II.214

839. OVOIDEUM, Thw.

II. 215

840. REVOLUTUM, Thw.

II.215

841. ORBICULARE, Thw.

II. 215

842. PROCERUM, Thw.

II. 216

843. CUNEATUM, Thw.

II. 216

844. umbellatum,- Burm. f. Kora-kaha, S.

Kaya, Kurrekaya, Pandikaya, T.

II.216 var. $\beta$ Thwaitesii, Clarke.

var. $\gamma$ rubro-coruleum, Clarke.

845. ClaRKEANUM, Cogn.

II. 217

846. discolor, Cogn.

II. 218

847. FUSCESCENS, Thw.

848. rostratum, Thw. Kuretiya, Hin-kure-

II.218

tiya, S.

849. angustifolium, Wight.

850. PHYLLANTHIFOLIUM, Thw.

851. RHINOPHYLLUM, Thw.

II.218

II.219

II. 219

II. 219

852. LEUCANTHUM, ThW.

II. 220

853. MACROCARPUM, ThW.

854. lævigatum, Bl.

II. 220

855. grande, Retz. Dodan-wenna, Dedikaha, S.

II. 220 var. $\beta$ ovatum, Clarke.

856. CAPITFillatum, L. Weli-kaha, Dodan-

kaha, S. Kattikaya, Venkalikaya, Pavaddai-

kaya, T.

II.222, Pl. XLI.

54. Lythraceæ.

309. Ammannia, L.

857. peploides, Spreng.

II. 223

858. Rotala, F. Muell.

II. 224

859. pentandra, Roxb.

II. 224

860. baccifera, L.

II. 224

861. cordata, W. \& A.

II. 225

862. lanceolata, Heyne.

II. 225

863. octandra, L. f.

II. 225

310. Woodfordia, Salisb.

864. floribunda, Salisb. Malitta, S.

II. 226

311. Pemphis, Forst.

865. acidula, Forst.

II. 227

312. Lawsonia, L.

866. alba, Lam. Marutonti, 'I'. Henna, Tree Mignonette. 
313. Lagerstrœmia, $\mathrm{L}$.

867. Flos-reginæ, Ret\%. Muruta, S. $\quad 11.228$

314. Sonneratia, L. f.

868. apetala, Ham. $\quad$ II.229

869. alba, Smith. II.230

870. acida, L. f. Kirilla, S. Kinnai, T. II.230

315. Axinandra, Thw.

871. ZEYLANICA, Thw. Kekiri-wara, S.

II. 231

55. Onagraceæ.

316. Jussiæa, L.

872. repens, L. Beru-diyamilla, S.

II. 233

873. suffruticosa, $\mathrm{L}$.

II, 233 var. $\beta$ subglabra, Thw.

317. Ludwigia, $\mathrm{L}$.

874. parviflora, Roxb.

II.234

875. prostrata, Roxb.

II. 234

318. Trapa, L.

876. bispinosa, Roxb. Ikiliya, S.

II. 235

56. Samydaceæ.

319. Casearia, Jacq.

877. esculenta, Roxb. Wal-waraka, S. Kakkuipalai, $\mathbf{T}$.

878. CORIACEA, Thw.

879. tomentosa, Roxb.

II. 237

II. 237

II. 238

320. Osmelia, Thw.

880. GARDNERI, Thw.

II. 238

321. Homalium, Jacq.

881. zeylanicum, Benth. Liyan, Liyangu, S. II.239

\section{Passifloraceæ.}

322. Modecca, Lam.

882. Wightiana, Wall.

II. 240

883. palmata, Lam. Hondala, Potu-honda, S. II.241

\section{Cucurbitaceæ.}

323. Trichosanthes, L.

884. palmata, Roxb. Titta-hondala, S.

var. $\beta$ tomentosa, Heyue.

885. nervifolia, $\mathrm{L}$.

I1. 244

886. cucumerina, L. Dummella, S. Pudal, T. II.245 var. $\beta$ laciniosa, Thw.

887. INTEGrifolia, 'Thw. 
324. Gymnopetalum, Arn.

888. Wightii, Arn.

II.246, PJ. XLIII.

var. $\beta$ zeylanicum, Arn.

325. Cephalandia, Schrad.

889. indica, Naud. Kowakka, S. Kovvai, 'T. II.247

326. Momordica, L.

*890. Charantia, L. Karivila, Batu-karivila, S.

Pakal, Nuti-pakal, T.

891. dioica, Roxb. Tumba-karivila. S.

Tumpai, Palu-pakal, T.

892. DENUdATA, Clarke.

327. Cucumis, $\mathrm{L}$.

893. trigonus, Roxb. Metukku, T.

894. pubescens, Willd. Gon-kekiri, Kekiri, S.

328. Luffa, Cav.

*895. ægyptiaca, Mill. Niyan-weta-kolu, S. Pikku, Pichukku, T.

II. 251

896. acutangula, Roxb. Weta-kolu, I)arkwelakolu, S. Peypichukku, T.

1I. 252 var. $\beta$ amara, Roxb.

329. Citrullus, Schrad.

897. Colocynthis, Schrad. Yakkomadu, S.

Peykkomaddi, T. Colocynth.

II. 253

330. Bryonia, L.

898. laciniosa, $\mathrm{L}$.

II. 254

331. Mukia, Arn.

899. scabrella, Arn. Hin-kekiri, S. Mochumochulkai, T.

900. leiosperma, Wight.

II. 254

II. 255

332. Zehneria, Endl.

90I. Hookeriana, Arn.

II. 256

902. hastata, Miq. Kawudu-kekiri, S. Peyppudal, T.

II. 256

333. Melothria, L.

903. ZEYLANICA, Clarke.

II. 257

334. Rhynchocarpa, Schrad.

904. rostrata, Naud.

II. 258

335. Corallocarpus, Welw.

905. epigæus, Clarke. Gopalanga, S.

II. 258

336. Cerasiocarpum, Hk. $\mathrm{f}$.

906. zeylanicum, Clarke.

II.259, Pl. XLIV.

337. Ctenolepis, Hk. f.

907. Garcini, Clarke. Mochu-mochukkai, T. 
338. Gynostemma, Bl.

908. laxa, Cogn.

II.260. PJ. XJV.

339. Zanonia, L.

909. indica, L. Wal-rasakinda, S.

I1.26]

59. Begoniaceæ.

340. Begonia, L.

910. cordifolia, Thw. Gal-ambala, S. II.262

911. TEnera, Dryand.

II. 263

912. THWAITESII, Hook.

II. 264

913. subpeltata, Wight.

I1. 264

914. malabarica, Lam. Hak-aimbala, S.

II. 264

60. Datiscaceæ.

341. Tetrameles, Br.

915. nudiflora, R. Br. Nigunu, Mugunu, S. II.265

61. Cactaceæ.

342. Rhipsalis, Gærtn.

916. Cassytha, Grertn. Wal-nawahandi, S. Il.266

\section{Ficoideæ.}

343. Sesuvium, L.

917. Portulacastrum, L. Vankiruvalai, T. II.26s

344. Trianthema, L.

918. monogyna, L. Hin-sarana, S.

919. triquetra, Rottl. \& Willd. Chirup-padikkirai, $\mathrm{T}$. var. $\beta$ Rottleri, Trim.

920. decandra, L. Maha-sarana, S. C'haranui, $\mathrm{T}$.

II. 270

345. Mollugo, L.

921. hirta, Thunb.

922. oppositifolia, L. Hin-pala, S. Kachchantirai, $\mathrm{T}$.

II. 269

II. 269

923. pentaphylla, L.

924. Cerviana, Ser. Pat-padakam, T.

II.270

925. disticha, Ser.

926. nudicaulis, Lam.

II.271

II. 271

II. 272

II. 272

II. 272

346. Gisekia, L.

927. pharnaceoides, L. Manali, 'T. 


\section{Umbelliferæ.}

347. Hydrocotyle, L.

928. javanica, Thunb. Mahu-yolukola, S. II.275

929. rotundifolia, Roxb. II.275

930. asiatica, L. Hin-gotukola, S. Vallarai, T. II.276

348. Sanicula, L.

931. europæa, L.

II. 276

349. Bupleurum, L.

932. virgatum, W. \& A. Wal-enduru, S.

350. Carum, L.

933. stictocarpum, Clarke.

II .278

351. Pimpinella, $\mathrm{L}$.

934. Heyneana, Wall. Wal-risamodagan, S. II.279

935. Leschenaultii, DC'.

II. 279

3อ̃2. Peucedanum, L.

936. zeylanicum, Gardn.

II. 280

353. Heracleum, L.

937. zeylanicum, Gardn.

II. 280

\section{Araliaceæ.}

354. Polyscias, Forst.

938. acuminata, Seem.

II. 282

355. Heptapleurum, Gærtn.

939. racemosum, Bedd.

940. stellatum, Gærtn. Itta, Itta-wel, S.

II. 283

941. exaltatum, Seem.

II. 283

942. EMARGINATUM, Seem.

II. 284

II.2S4, Pl. XLVI.

\section{Cornaceæ.}

356. Alangium, Lam.

943. Lamarckii, Thw. Mul-anninchil, T. II.285

944. GLANDULOSUM, Thw. II.286

357. Mastixia, Bl.

945. TETrANDRA, Clarke. Maha-tawara, S.

II.287, PI. XLVII.

var. $\beta$ Thwaitesii, Clarke. Diya-taleya, $\mathrm{S}$.

946. arborea, Clarke.

II. 287

\section{Caprifoliaceæ.}

358. Viburnum, L.

947. coriaceum, Bl.

var. $\beta$ capitellata, Clarke.

948. erubescens, Wall. 
67. Rubiaceæ.

359. Sarcocephalus, Afzel.

949. cordatus, Miq. Bakmi, S. Vammi, 'T. II.292

360. Anthocephalus, A. Ricb.

950. Cadamba, Miq. Embul-bakmi, S. Vellaikadampa, T.

II. 293

361. Adina, Salisb.

951. cordifolia, Hk. f. Kolon, S. Manchalkadampa, Raja-murunkai, T.

II.293

362. Stephegyne, Korth.

952. parvifolia, Kortb. Chelampai, Nirkadampa, T.

953. tubulosa, Hk. f. Helamba, S.

II. 294

II. 295 var. $\beta$ minor, Thw.

363. Nauclea, L.

954. ZEYLANICA, Hk. f.

II.296

364. Uncaria, Schreb.

955. dasyoneura, Korth́. var, Thwąitesii, Hk. f.

II. 296

365. Wendlandia, Bartl.

956. Notoniana, Wall. Rawanidala, S. var. $\beta$ zeylanica, Hk. $\mathrm{f}$.

II. 297

366. Dentella, Forst.

957. repens, Forst.

II.298

367. Neurocalyx, Hook.

958. zEYLANICUS, Hook.

II. 299

959. Wightii, Arn.

II. 299

960. GARDNERI, Thw.

II. 300

961. CHAMPIONII, Benth.

II. 300

368. Alloophania, Thw.

962. DECIPIENS, Thw.

var. $\beta$ flavescens, 'l'hw.

var. " Ainottii, Hk. f.

II.301. Pl, Xr,VIII.

369. Fergusonia, Hk. f.

963. zeylanica, Hk. f.

II.302

370. Hedyotis, L.

964. fruticosa, L. Weraniya, S.

II. 304

965. EvenIa, Thw.

II.304

966. CYMOSA, Thw.

II.305

967. MACR EI, Hk. f.

II.305

968. овScuka, Thw.

II. 305

var. $\beta$ minor, Thw.

969. COPROsmoIDhs, Trim. 
970. Membranacea, Thw.

II. 306

971. THWAITESII, Hk. f.

II.307

972. NodULOSA, Arn.

II.307

var. $\beta$ Walkeri, Hk. f.

973. CINEREO-VIRIDIS, Thw.

II. 308

var. $\beta$ subverticillata, Trim.

var. $\gamma$ fumata, Thw.

974. RHINophylLA, Thw.

II.308

975. Lessertiana, Am.

II.309, Pl. XLIX.

var. $\beta$ pilosa, Thw.

var. $\gamma$ confertiflora, Thw.

var. ô marginata, Thw.

var. $\&$ flavescens, Thw.

976. QUINQUENERVIA, Thw.

II. 310

977. GARDNERI, Thw.

II. 310

978. LAWSONI

II. 310

979. verticillaris, W. \& A.

II. 311

980. cærulea, W. \& A.

981. nitida, W. \& A. Pita-sudu-pala, S.

II.312

982. Auricularia, L. Geta-kola, S.

II.312

983. inamena, Thw.

II. 313

984. OYANESCENS, Thw.

II.313

II. 313

371. Oldenlandia, L.

985. corymbosa, L. Wal-patpardagrem, S. II.314 var. $\beta$ racemosa, Thw.

986. diffusa, Roxb.

II. 315

987. herbacea, Roxb.

II.315

988. umbellata, L. Saya, S. Chaya, 'T.

Chay-root.

989. trinervia, Retz.

990. stricta, $\mathrm{L}$.

II.316

991. biflora, $\mathrm{L}$.

II.316

II.317

372. Anotis, DC.

992. quadrilocularis, Hk. f.

993. NUMMULARIA, Hk. f.

II.318

994. NUMMULARIFORMIS, Trim.

II.318

995. RICHARDIANA, Hk. f.

II.319

II.319

373. Ophiorrhiza, L.

996. Mungos, L. Dat-ketiya, Wal-ekaweriya, S. II.320 var. $\beta$ nemorosa, Hk. f.

var. $\gamma$ angustifolia, Hk. f.

997. Harrisiana, Heyne.

998. radiCANS, Gardn.

II.321

999. pectinata, Arn.

II.322

1000. PAlLIDA, Thw.

II.322

1001. ALECHOMIFOLIA, Thw. 
374. Mussænda, $\mathrm{L}$.

1002. frondosa, L. Mussenda, Wel-but-sarana, $\mathrm{S}$. var. $\beta$ glabrata, Hk. $\mathrm{f}$.

375. Acranthera, Arn.

1003. zeylanica, Arn.

376. Leucocodon, Gardn.

1004. RETiCULATUM, Gardn.

377. Urophyllum, Wall.

1005. ELLIPTICUM, Thw.

II. 326

1006. ZEYLANICUM, Thw.

II.326

378. Schizostigma, Arn.

1007. HIRSUTUM, Arn.

379. Webera, Schreb.

1008. corymbosa, Willd. Tarana, S. Karanai, T.

II.328 var. $\beta$ montana, Thw.

380. Byrsophyllum, Hk. f.

1009. ELLIPTICUM, Bedd.

II.329

381. Randia, L.

1010. uliginosa, DC. Et-kukuruman, Wadiga, S. II.330

1011. dumetorum, Lam. Kukuruman, S. Karai, $\mathrm{T}$.

1012. malabarica, Lam. Pudan, T.

II. 330

1013. GARDNERI, Hk. f.

II.331

1014. rugulosa, Hk. f.

II. 331

II.331

382. Gardenia, L.

1015. latifolia, Ait. Galis, S.

1016. coronaria, Ham.

II.332

1017. turgida, Roxb.

II.333

II.333

383. NARGEDIA, Bedd.

1018. MACROCARPA, Bedd.

II. 334

384. ScyPHOSTACHYs, Thw.

1019. PEDUNCULATUS, Thw.

1020. COFF AOIDES, Thw. Wal-kopi, S. Wild Coffee.

II.335

II.335

385. Diplospora, DC.

1021. DALZELLII, Hk. f. Vella, T. II.336, Pl. L. 1022. ERYTHROSPORA, Bedd.

II.336

386. Scyphiphora, Gærtn.

1023. hydrophylacea, Gærtn. f.

387. Guettarda, L.

1024. speciosa, L. Nil-pichcha, S. Panir, T. II.33s 
388. Timonius, DC.

1025. Jambosella,Thw. Peddimella, Angana, S. II.338

389. Dichilanthe, Thw.

1026. zeyLanica, Thw.

Il.339

390. Knoxia, L.

1027. corymbosa, Willd.

II. 340

1028. mollis, W. \& A.

II. 340

1029. ZEYLANICA, L.

II.341

1030. Platycarpa, Arn.

var. $\beta$ hirsuta, Thw.

var. $\gamma$ foliosa, Thw.

var. $\delta$ spicata, Thw.

391. Canthium, Lam.

1031. didymum, Gærtn, f. Pana-karawu, Galkaranda, Panduru, S. Vatchikuran, Yerkoli,

T. Ceylon Boxwood.

II.343

var. $\beta$ lanceolatum, Thw.

1032. MoNTANUM, Thw.

II.343

var. $\beta$ minus, Thw.

1033. PUBERULUM, Thw.

II.344

1034. Rheedii, DC. var. $\beta$ minus, Thw.

II.344

1035. MACROCARPUM, Thw.

1036. CaMPaNULATUM, Thw.

II.345

1037. parviflorum, Lam. Kara, S. Karai, T. II.346

392. Ixora, L.

1038. CaLYCINA, Thw.

II. 347

1039. ThWAITESII, Hk. $f$.

II. 347

var. $\beta$ velutina, Thw.

1040. parviflora, Vahl. Maha-ratambala, S.

Karankutti, Painkuray, Kanmuttankirai, T. II.348 var. $\beta$ zeylanica, Hk. $f$.

1041. JUCUNDA, Thw.

II.348, Pl. LII.

1042. coccinea, L. Ratambala, S. Vedchi, T. II.348

393. Pavetta, L.

1043. indica, L. Pawatta, S. Pavaddai, T. II.349 var. $\beta$ montana, Thw.

1044. hispidula, W. \& A.

II. 350

1045. ANGUSTIFolia, Thw.

II.350

1046. Gleniei, Thw. Vetpavaddai, T.

II.351

1047. INVOLUCRATA, Thw.

II.351

394. Coffea, L.

1048. Wightiana, Wall. Kaddumallikai, T. II.352 1049. travancorensis, W. \& A. II.353, P1. LIII. 
395. Morinda, L.

1050. tinctoria, Roxb. Ahu, S. Manchavanna, $\mathrm{T}$.

1051. citrifolia, L. $A h u, \mathrm{~S}$.

II.354

1052. umbellata, L. Kiri-wel, Maha-kiri-wel, S.

II.355

396. Prismatomeris, Thw.

1053. albidiflora, Thw.

II.355

var. $\beta$ Fergusonii, Trim.

397. Psychotria, L.

1054. STENOPHYLLA, Hk. f.

II. 357

1055. GLANDULIFERA, Thw.

II.357

1056. GARDNERI, Hk. f.

II. 358

1057. Thwaitesii, Hk. f.

II. 358

var. $\beta$ coronata, Hk. $f$.

1058. WIGHTIANA, Hk. $f$.

II.358 var. $\beta$ affinis, Hk. $f$.

1059. elongata, Hk. f.

II. 359

1060. sarmentosa, Bl. Wal-gonika, S.

II. 359

1061. MOONII, Hk. f.

II. 360

1062. SORDIDA, Thw.

II.360

1063. LONGEPETIOLATA, Thw.

II.361

1064. PLURIVENIA, Thw.

II.361

1065. FILIPES, Hk. f.

II.361

1066. bisulcata, W. \& A.

II.362, Pl. LIV.

398. Chasalia, Comm.

1067. curviflora, Thw.

II. 362

399. Geophila, Don.

1068. reniformis, D. Don. Agukarni, S.

II. 363 400. Lasianthus, Jack.

1069. MooNII, Wight.

1070. THWAITESII, Hook. $\mathrm{f}$.

II.364

II.365 var. $\beta$ nitidus, Thw.

1071. RHINOPHYLLUS, Thw.

II.365

1072. WALKERIANUS, Wight.

II.365

1073. GARDNERI, Hk. f.

II.366

1074. OLIGANTHUS, Thw.

II.366

1075. obliquUs, Thw.

II.367

1076. STRIGOSUS, Wight.

II.367

var. $\beta$ protractus, Hk. f.

1077. VARIANS, Thw.

II.368

401. Saprosma, Bl.

1078. indicum, Dalz.

II.368

1079. SCABRIDUM, Bedd.

II.369

1080. zeylanicum, Bedd.

II.369 
402. Hydrophylax, L. f.

1081. maritima, L. f. Mudu-geta-kola, S.

II.370

403. Spermacoce, L.

1082. stricta, L. f.

II. 371

1083. ocymoides, Burm. f.

II.371

1084. hispida, L. Hin-geta-kola, S. Yar, Nattaichchuri, T.

II.371

404. Rubia, L.

1085. cordifolia, L. Manda-madini-wel, Yoganawel, S.

II.372

405. Galium, L.

1086. asperifolium, Wall.

II.373

68. Valerianaceæ.

406. Valeriana, L.

1087. MOONII, Arn.

III.1

69. Dipsacaceæ.

407. Dipsacus, L.

1088. WALKERI, Arn.

III. 2

70. Compositæ.

408. Vernonia, Schreb.

1089. GARDNERI, Thw.

III. 6

1090. THWAITESII, Clarke.

III. 6

1091. ANCEPS, Clarke.

1092. cinerea, Less. Monara-kudimbiya, S.

Chitiviyarchenkalainir, T.

1093. SETIGERA, Arn.

III. 6

1094. HOOKERIANA, Arn.

1095. SCARIOSA, Arn.

var. $\beta$ crassa, Thw.

*1096. anthelmintica, Willd. Sanninayan, S.

Kadduchchirakam, T.

III.9

III.9

1097. NEMORALIS, Thw.

1098. Wightiana, Arn.

1099. zerlanica, Less. Pupula, Hin-botiya,

S. Kuppilay, T.

III.9

1100. pectiniformis, DC.

1101. arborea, Ham. Kobomella, S.

III. 7

III.7

III. 8

III. 8

III.10

III.10

III.11

409. Elephantopus, L.

1102. scabe1, L. Et-adi, S. Anichovadi, T. III.12

410. Adenostemma, Forst.

1103. viscosum, Forst.

III. 13

var. $\beta$ reticulatum, Clarke. 
411. Dichrocephala, DC.

1104. latifolia, DC.

III.14

412. Grangea, Adans.

1105. maderaspatana, Poiret.

III.14

413. Myriactis, Less.

1106. Wightii, DC.

III.15

414: Lagenophora, Cass.

1107. Billardieri, Cass. III.16, Pl. LV.

415. Erigeron, L.

1108. asteroides, Roxb. Narakaramba, T. III.16

416. Microglossa, DC.

1109. zeylanica, Clarke. Pupula, S.

III.17

417. Conyza, Less.

1110. viscidula, Wall.

III.18

418. Blumea, DC.

1111. amplectens, DC.

III.19 var. $\beta$ arenaria, Hk. $\mathrm{f}$.

1112. bifoliata, DC.

III.19

1113. lacera, DC.

III.19

1114. barbata, DC.

III. 20

1115. flexuosa, Clarke.

III. 20

1116. CRINITA, Arn.

III. 21

1117. hieraciifolia, DC.

III.21

1118. membranacea, DC.

III.22 var. $\beta$ Gardneri, Hk. $\mathrm{f}$

1119. spectabilis, DC.

III.22

1120. aNgustifolia, Thw.

III.23, Pl. LVI.

419. Laggera, Schultz-Bip.

1121. alata, Sch.-Bip.

III.23

1122. aurita, Benth.

III. 24

420. Epaltes, Cass.

1123. divaricata, Cass. Hin-muda-mahana, S. III.24

421. Sphæranthus, L.

1124. amaranthoides, Burm. f. Chiva-charantai, $\mathrm{T}$.

1125. indicus, L. Muda-mahana, S.

III. 25

1126. africanus, $\mathrm{L}$.

III. 26

III.26

422. Blepharispermum, Wight.

1127. petiolare, DC.

III. 27

423. Anaphalis, DC.

1128. cinnamomea, Clarke.

III.28

1129. Pelliculata, Trim.

III.28, Pl. LVII.

$6(11) 10$ 
1130. FRUTICOSA, Hk. f.

1131. thWAITESII, Clarke.

III. 29

1132. oblonga, DC.

III. 29

1133. zEYLANICA, Clarke.

III. 30

1134. marcescens, Clarke.

III.30

var. $\beta$ sulphurea, Trim.

1135. brevifolia, DC.

III.31

III.3I

424. Helichrysum, Gærtn.

1136. buddleoides, DC.

III.32

425. Vicoa, Cass.

1137. auriculata, Cass. Ran-hiriya, S.

III.33

426. Chrysogonum, L.

1138. heterophyllum, Clarke.

III.34

427. Xanthium, L.

1139. Strumarium, L.

III.35

428. Siegesbeckia, L.

1140. orientalis, $L$.

III.36

429. Eclipta, L.

1141. alba, Hassk. Kikirindi, S. Karippan, T.

III.37

430. Blainvillea, Cass.

1142. latifolia, DC.

III.37

431. Wedelia, Jacq.

1143. calendulacea, Less. Ran-wan-kikirindi, S. III.38

1144. biflora, DC.

III.39

432. Spilanthes, Jacq.

1145. Acmella, L. Akmella, S. Toothache plant.

III.40

433. Bidens, L.

*1146. pilosa, L. Wal-te-kola, S. Spanish needle.

III.40 var. $\beta$ bipinnata, Hk. f.

434. Glossogyne, Cass.

1147. pinnatifida, DC.

III.41

435. Centipeda, Lour.

1148. orbicularis, Lour. Wisaduli, S.

III.42

436. Artemisia, L.

*1149. vulgaris, L. Wal-kolondu, S. Mugwort. III.43

437. Gynura, Cass.

1150. lycopersicifolia, DC.

III.43

1151. ZeYLANICA, Trim.

III.44, P1. LVIII.

1152. HISPIDA, Thw. 
438. Enulia, Cass.

1153. sonchifolia, DC. Kadupara, S.

1154. zeYLANICA, Clarke. var. $\beta$ Walkeri, Trim.

439. Notonia, DC.

1155. grandiflora, DC.

1156. Walkeri, Clarke.

440. Senecio, L.

1157. gracilis, Arn.

1158. GARDNERI, Clarke.

III.48

1159. ludens, Clarke.

III. 48

1160. Walkeri, Arn.

III. 49

1161. corymbosus, Wall.

III.49

1162. scandens, D. Don.

III.50

III.50

441. Crepis, L.

1163. japonica, Benth.

III.5I

1164. fuscipappa, Clarke.

III.51

442. Lactuca, L.

1165. Heyneana, DC.

III. 52

443. Launæa, Cass.

1166. pinnatifida, Cass.

III.52

\section{Stylidiaceæ.}

444. Stylidium, Sw.

1167. uliginosum, Sw.

III.5.3

72. Goodenoviaceæ.

445. Scævola, L.

1168. Kœnigii, Vahl. Takkada, S.

III. 54

1169. Plumieri, Vahl. Hin-takkada, S.

III. 55

73. Campanulaceæ.

446. Lobelia, L.

1170. zeylanica, $\mathrm{L}$. var. $\beta$ Walkeri, Clarke.

1171. trigona, Roxb.

III.56

1172. affinis, Wall.

III.56

III.57

1173. nicotianæfolia, Heyne. Rasni, S.

III.57 var. $\beta$ trichandra (Wight), Trim.

447. Wahlenbergia, Schrad.

1174. gracilis, A. DC. Hare-bell.

III.58

448. Sphenoclea, Grortn.

1175. zeylanica, Gærtn. 
449. Campanula, L.

1176. cancscens, Wall.

III. 60

1177. fulgens, Wall.

III. 60

74. Vacciniaceæ.

450. Vaccinium, L.

1178. Leschenaultii, Wight. Boralu, S.

III.61

75. Erlcaceæ.

451. Gaultheria, L.

1179. fragrantissima, Wall. Wel-kapuru, S.

III. 62 var. $\beta$ hirsuta, Gardn.

452. Rhododendron, $\mathrm{L}$.

1180. arboreum, Sm. Ma-ratmal, S.

III.63

\section{Plumbaginaceæ.}

453. Plumbago, $\mathrm{L}$.

1181. zeylanica, L. Ela-netul, S.

III. 65

77. Primulaceæ.

454. Lysimachia, L.

1182. ramosa, Wall.

III.66

1183. deltoidea, Wight.

III. 66

78. Myrsinaceæ.

455. Mæsa, Forsk.

1184. indica, A. DC. (Perrotetiana, A. DC.) Matabimbiya, S.

III. 67

456. Myrsine, L.

1185. capitellata, Wall. ${ }^{1}$

var. $\beta$ lanceolata, Clarke.

var. $\gamma$ sessiliflora, Thw.

457. Embelia, Burm. $f$.

1186. Ribes, Burm. f. Wel-embilla, S.

III. 69

11 77. robusta, Roxb. (tsjeriam-cottam, A. DC.) III.70

1188. viridiflora, Scheff. (basaal, A. DC.) III.70

458. Ardisia, $\mathrm{Sw} .^{2}$

1189. Missionis, Wall.

III.71

1190. willisis, Mez. (A. humilis, Trim. pp.)

Lunu-dan, S. (Gardner, 516, Hügel, 3,581,

C. P., 2,829, \&e.)

1 Mez, in Das Pflanzenreich, splits this into five species, Thwaitesii, ceylanica, robusta, oxigua, and rubens, and transfors it to Rapanea.

${ }^{2} \mathrm{Cf}$. Mez in Das Pflanzenreich. 


\section{( 51$)$}

1191. humilis, Vahl. (incl. A. elliptica, Thunb.)

Balu-dan, S. var. $\beta$ Wightiana, A. DC.

1192. solanacea, Roxb.

1193. Gardneri, Clarke.

var. $\beta$ zeylanica, Clarke.

1194. pauciflora, Heyne.

III. 73

1195. POLYLEPIS, Mez. (last sp. p.p.)

l196. MOONII, Clarke.

III.73

459. Egiceras, Gærtn.

1197. majus, Gærtn. Hin-kadol, S. Vitlikanna, T.

III.74

\section{Sapotaceæ.}

460. Chrysophyllum, L.

1198. Roxburghii, G. Don. Lawulu, S.

III. 76

461. Sideroxylon, L.

1199. tomentosum, Roxb. Mul-makil, T.

III.77

462. Isonandra, Wight.

1200. lanceolata, Wight. Kiri-varala, MIolpedda, S.

$$
\begin{aligned}
& \text { var. } \beta \text { angustata, Thw. } \\
& \text { var. } \gamma \text { montana, Thw. } \\
& \text { var. } \delta \text { compta, Thw. } \\
& \text { var. } \varepsilon \text { major, Clarke. }
\end{aligned}
$$

463. Bassia, Kœnig.

1201. longifolia, L. Mi, S. Iluppai, T.

1202. MOONII, Bedd.

III.79

1203. NERITFOLIA, Moon. Gan-mi, S. III.80, Pl. LIX.

1204. MICROPHYLLA, Hook.

1205. Fulva, Bedd. Wana-mi, S.

III. 80

III.81

464. Palaquium, Blanco.

1206. Petiolare, Engl. Molpedda, S.

III.82

1207. GRANDE, Engl. Kirihiriya, Mihiriya, Kirihembiliya, Molpedda, S.

III. 82 var. $\beta$ parvifolium, Clarke.

var. $\gamma$ angustatum, Trim.

1208. Rubigrinosum, Engl.

III. 83

1209. oanaliculatum, Engl.

III. 84

1210. ThWAItesir, Trim.

III.84

1211. LAVIfolium, Engl.

III. 84

1212. PAUCIFLORUM, Engl.

III.85 
465. Mimusops, L.

1213. Elengi, L. Muna-mal, S. Makil, Mukulai, Vilva-padri, $\mathrm{T}$.

1214. hexandra, Roxb. Palu, S. Palai, T. III.86

\section{Ebenaceæ.}

466. Maba, ${ }^{5}$ Forst.

1215. ACUMINATA, Hiern.

1216. OVALIFOLIA, Hiern.

1217. OBLONGIFOLIA, Hiern.

1218. buxifolia, Pers. Kalu-habaraliya, S. Juvarai, Irumpalai, $\mathbf{T}$. var. $\beta$ microphylla, Thw. var. $\gamma$ Ebenus, Thw. var. $\delta$ angustifolia, Thw.

467. Diospyros, L. ${ }^{1}$

1219. ovalifolia, Wight. Kunumella, Habara,

S. Vedukkanari, $\mathrm{T}$.

1220. montana, Roxb. Mulkarunkali, Katukanni, Vakkana, T.

III. 89

III.91

III.92

1221. Embryopteris, Pers. Timbiri, S. Panichchai, $\mathrm{T}$.

var. $\beta$ atrata, Thw.

var. $\gamma$ nervosa, Thw.

1222. Toposia, Ham. Kahakala, Kaluwella, S.

Vellai Thoveri, T.

1223. Ebenum, Kœnig. Kaluwara, S. Karunkali, T. Ebony.

1224. pruriens, Dalz.

1225. ATTENUATA, Thw. Kadumberiya, $\mathrm{S}$.

1226. ACUTA, Thw.

III.93

1227. GARDNERI, Thw. Kadumberiya, Kallu,

S. Bastard ebony.

1228. oocarpa, Thw. Kalu-kadumberiya, Etatimbiri, S. Vellai-karunkali. T.

III.94

III.95

III.96

III.96

1229. QUÆSITA, Thw. Kalumediriya, S.

Calamander.

III.96

III.97

III.97

1230. sylvatica, Roxb. Sudu-kadumberiya, S.

Karuppu-thoveri, T.

III.98

1231. Melanoxylon, Roxb. Kadumberiya, S. III.99

1232. HIRSUTA, L.'f.

1233. insignis, Thw. Gona, Poruwa-mura, Wal-mediriya, S.

1234. OPPOSITIFOLIA, Thw. Kalumediriya, Kadumberiya, S. 
1235. ThwaItesII, Bedd. Kadumberiya, S. III.101 1236. MooNII, Thw. Kadumberiya, Kaluwella, S.

1237. affinis, Thw. Kaluwella, S. Semelpanachai, $\mathrm{T}$.

1238. crumenata, Thw.

III.101

III. 102

III.102

\section{Styraceæ.}

468. Symplocos, L. ${ }^{1}$

1239. spicata, Roxb. Bombu, Wal-bombu, S. III.104

1240. furcata, Brand. (obtusa, Wall.) var. $\beta$ major, Thw. var. $\gamma$ obovata, Thw. var. \& cucullata, Thw.

1241. LÆTA, Thw.

1242. BRACTEALIS, Thw.

1243. VERSICOLOR, Clarke (spicata according to Brand.)

1244. ACUTA, Thw.

1245. CUNEATA, Thw.

1246. HISPIDULA, Thw.

1247. WALKERI, Brand.

1248. JUCUXDA, Thw.

1249. angustata, Clarke.

1250. LATIFLORA, Clarke.

1251. ELEGANS, Thw.

III. 105

III.106

1252. MINOR, Clarkt. var. $\beta$ glabrescens, Thw.

1253. HEBANTHA, Thw.

IIII.106

III.106

III. 107

IFI.107

1254. CORDIFOLIA, Thw.

1255. APICALIS, Thw. var. $\beta$ glabrifolia, Thw.

III. 107

III.108

III.108

III. 108

III.109

1256. MargiNaLIS, Thw.

III. 109

III.110

III.110

1257. coronata, Thw.

1258. pendula, Wight. (pauciflora, Wight.)

FII.111

III.111

III.1.11

\section{Oleaceæ.}

469. Jasminum, L.

1259. glabriusculum, B1.

III.113

1260. sessiliflorum, Vahl.

III.114

1261. angustifolium, Vahl. Wal-pichcha, S.

III.114

1262. auriculatum, Valil.

III.115

1263. flexile, Vahl.

III.115

1264. humile, L.

III.115

1 See Brand, in Das Pflanzenroich. 
470. Linociera, Sw.

1265. PURPUREA, Vahl. Geriata, S. Kattimuruchan, $\mathrm{T}$.

1266. albidiflora, Clarke.

III.116 var. $\beta$ rostrata, Clarke.

1267. leprocarpa, Clarke.

III.117

III. 117

471. Olea, L.

1268. glandulifera, Wall.

III.118

1269. polygama, Wight.

III.118

472. Ligustrum, L.

1270. Walkeri, Dene.

III.119

83. Salvadoraceæ.

473. Salvadora, L.

1271. persica, L. Uvay, Viyay, T.

III. 120

474. Azima, Lam.

1272. tetracantha, Lam. Iyanku, Ichanku, T. 1II.121

\section{Apocynaceæ.}

475. Willughbeia, Roxb.

1273. ZEYLANICA, Thw. Kiri-gedi, Kiri-wel, S. III.123

476. Carissa, L.

1274. Carandas, L. Maha-karamba, S. Perunkila, $\mathrm{T}$.

III.124

1275. spinarum, L. Hin-karamba, S. Chirukila, Kilatti, T.

III. 125

477. Rauvolfia, L.

1276. serpentina, Hk. f. Ekaweriya, Ratekaweriya, S.

1277. densiflora, Hk. f.

III. 126

III. 126

478. Alyxia, Br.

1278. zeYLANICA, Wight. Walkaduru, S.

III. 127

479. Hunteria, Roxb.

1279. corymbosa, Roxb. Mediya, S.

III.128

480. Cerbera, L.

1280. Odollam, Gærtn. Gon-kaduru, S.

III. 128

481. Ochrosia, Juss.

1281. borbonica, Gmel. Mudu-kaduru, S. III.129,

Pl. LX.

482. Vinca, L.

1282. pusilla, Murr.

III. 130

483. Holarrhena, Br.

1283. мıтIS, Br. Kiri-walla, Kiri-mawara, S. III.131 
484. Tabernæmontana, L.

1284. dichotoma, Roxb. Divi-kaduru, S.

Eve's apple, Forbidden fruit.

III. 132

485. Alstonia, Br.

1285. scholaris, Br. Ruk-attana, Elilaippatur, $\mathrm{T}$.

III.133

486. Parsonsia, $\mathrm{Br}$.

1286. spiralis, Wall.

III. 134

487. Vallaris, Burm.

1287. Heynei, Spreng.

III.135

48s. Wrightia, Br.

1288. FLAVIDO-ROSEA, Trim. III.136, PI. LXI.

1289. ANGUSTIFOLIA, Thw.

III. 136

1290. tomentosa, Rœm. \& Sch. Palmadankai,

$\mathrm{T}$.

III. 137

1291. ZEYLANICA, Br. Wal-idda, Sudu-idda, S. III.137

489. Chonemorpha, G. Don.

1292. macrophylla, G. Don. Bu-wal-anguna, $\mathrm{S}$.

III.138

490. Aganosma, G. Don.

1293. cymosa, G. Don.

III. 139

491. Baissea, A. DC.

1294. ACUMINATA, Hk. f.

III. 140

492. Anodendron, A. DC.

1295. paniculatum, A. DC. Dul, As-wel, S. III.141

1296. RHINOSPORUM, Thw.

III.141

493. Ichnocarpus, Br.

1297. frutescens, Ait. Kiri-wel, S.

III.142

85. Asclepiadaceæ.

494. Hemidesmus, Br.

1298. indicus, Br. Iramusu, S. Nannari, T. III.144

495. Cryptolepis, Br.

1299. Buchanani, Rœm. \& Sch. Wel-rukattana, $\mathrm{S}$.

III.145

496. Secamone, Br.

1300. emetica, Br.

III. 146

497. Toxocarpus, W. \& A.

1301. Kleinii, W. \& A.

III. 146

498. Oxystelma, Br.

1302. esculentum, Br. Kulappalai, T. TII.147

$6(11) 10$ 
499. Calotropis, $\mathrm{Br}$.

1303. gigantea, Br. Wara, S. Manakkovi, Errukalai, Urkkovi, T.

III. 148

500. Pentatropis, Br.

1304. microphylla, W. \& A.

III. 149

501. Dæmia, Br.

1305. extensa, Br. Medahangu, S. Uttama-

kam, Veliparatti, T.

III. 150

502. Holostemma, Br.

13116. Rheedei, Wall.

III.150

503. Cynanchum, L.

1307. pauciflorum, Br. Kan-kumbala, S.

III.151

504. Sarcostemma, Br.

1308. Brunonianum, W. \& A. Muwa-kiriya,

S.

III. 152

505. Gymnema, Br.

1309. sylvestre, Br. Mas-bedde, $\mathrm{S}$.

III. 153

var. $\beta$ zeylanicum, Hk. $f$.

1310. Rotundatum, Thw.

III. 153

1311. lactiferum, Br. Kurinnan, S. and T. III.154 var. $\beta$ Thwaitesii, Hk. f.

1312. pergularioides, Wight. \& Gardn.

III. 154 var. $\beta$ Gardneri, Hk. f. var. $\gamma$ stenoloba, Hk. f. (sp.)

506. Marsdenia, $\mathrm{Br}$.

1313. tenacissima, Moon. Muruwa-dul, S.

III. 155

507. Tylophora, Br.

1314. fasciculata, Ham.

III. 156

1315. Iphisia, Done.

III. 157

1316. membranifolia, Thw.

III. 157

1317. zeylanica, Dene.

III. 157

1318. tenuis, B1.

III. 158

1319. CORDIFOLIA, Thw.

III. 158

1320. asthmatica, W. \& A. Bin-nuga, S. Peypalai, Nancharapanchan, T. Wild ipecacuanha.

III. 158

1321. FLAVA, Trim. Mudu-bin-nuga, S.

III. 159 ,

Pl. LXII.

508. Cosmostigma, Wight.

1322. racemosum, Wight.

III. 160

509. Dregea, E. Meyer.

1323. volubilis, Benth. Kiri-anguna, S. Kurincha, $\mathrm{T}$. 
510. Disehidia, Br.

1324. Nummularia, Br.

III.161

511. Hoya, Br.

1325. pauciflora, Wight.

III.162

1326. ovalifolia, W. \& A.

III. 162

512. Heterostemma, W. \& A.

1327. tanjorense, W. \& A.

III. 163

513. Leptadenia, $\mathrm{Br}$.

1328. reticulata, W. \& A. Palai, T. (?)

III.164

514. Ceropegia, L.

1329. elegans, Wall. var. $\beta$ Walkere (Wight.), Trim.

1330. GARDNERI, ThW.

III. 165

1331. Thwaitesii, Hook.

III.165

1332. Decaisneana, Wight.

III. 166

1333. biflora, L. Wel-mottu, S.

III. 166

1334. PARVIFLORA, Trim.

III. 167

III.167, Pl. LXIII.

515. Caralluma, Br.

1335. fimbriata, Wall. Mankalli, T.

1336. Campanulata, N. E. Br.

III. 168

III. 168

\section{Loganiaceæ.}

516. Mitrasacme, Lab.

1337. alsinoides, $\mathrm{Br}$.

III.170)

517. Fagræa, Thunb.

1338. zeylanica, Thunb. Etamburu, S.

1339. obovata, Wall.

III.170

III.171

var. $\beta$ Gardneri, Clarke.

518. Strychinos, L.

1340. MICRANTHa, Thw. Kachchalkodi, 'T. III.172

1341. colubrina, L. var. zeylanica, Clarke. III.173

1342. Beddomei, Clarke.

III. 173 var. $\beta$ coriacea, Clarke.

1343. Benthami, Clarke.

III. 174 var. $\beta$ parvifolia, Benth.

1344. CINNAMOMIFOLIA, Thw. Eta-kirindiwel, Wel-beli, S.

III. 174

1345. Nux-vomica, L. Goda-kaduru, S. Eddi, Kanchurai, 'T. Nux vomica.

1346. potaturum, L. f. Ingini, S. Tette, T. Cleuring-nut.

III. 175

III. 176 
519. Grertuera, Lam.

1347. Kønigii, Wight. Pera-lambula, s.

III. 177

var. $\beta$ thyrsiflora, Thw.

var. $\gamma$ divaricata, Clarke.

1348. ROSEA, Thw.

III. 177

1349. WALKERI, Wight.

III. 178 var. $\beta$ Gardneri, Clarke.

1350. TERNIFOLIA, Thw.

III.178, Pl. LXIV.

87. Gentianaceæ.

520. Exacum, L.

1351. AXILLARE, Thw.

III. 180

1352. Walkeri, Arn.

1353. zeylanicum, Roxb. Bindara, Ginihiriya, $\mathrm{S}$.

III. 180

var. $\beta$ pallidum, Trim.

var. $\gamma$ Ritigalense, Willis.

1354. macranthum, Arn.

III.181

1355. pedunculatum, L.

III.182

var. $\beta$ petiolare, Griseb.

1356. sessile, L. (? endemic.)

III. 183

521. Hoppea, Willd.

1357. fastigiata, Clarke.

III. 183

522. Canscora, Lam.

1358. diffusa, $\mathrm{Br}$.

III. 184

1359. sessiliflora, Roem. \& Schult.

III.184

1360. Roxburghii, Arn.

III. 185

1361. decussata, Roem. \& Schult.

III.185

523. Enicostema, B1.

1362. littorale, Bl. Vellaruku, T.

III. 185

524. G'entiana, L.

1363. quadrifaria, Bl.

III. 186

525. Crawfurdia, Wall.

1364. japonica, Sieb. \& Zucc. var. Championii, Clarke.

III.187, Pl. LXV.

526. Swertia, L.

1365. ZEYLANICA, Walker.

III. 187

527. Limnantlienum, Gmel.

1366. indicum, Thw. Olu, Maha-ambala, S. III.188

1367. cristatum, Griseb. Hin-ambala, S.

1368. 13arvifolium, Grisel). Bin-olu, S.

III. 189

1369. aurantiacum, Dalz.

III. 189

III. 190 
88. Hydrophyllaceæ.

528. Hydrolea, L.

1370. zeylanica, Valnl. Diya-kirilla, s'.

III.191

89. Boraginaceæ.

529. Cordia, L.

1371. Myxa, L. Lolu, S. Naruvili, Vidi, T.

Sebestens.

III. 193

var. $\beta$ obliqua, Willd. ( $s p$.

1372. monoica, Roxb. Naruvili, Pon-naruvili, $\mathbf{T}$.

1373. Rothii, Røem. \& Sch.

1374. OBLONGIFOLIa, Thw.

1375. subcordata, Lam.

III. 193

III. 194

III.194

III.195

530. Ehretia, L.

1376. lævis, Roxb. Addula, Chiru-pulichchul, T.

III. 195

1377. buxifolia, Roxb. Hin-tambala, S. Pakkuvetti, 'T.

III.196

531. Coldenia, L.

1378. procumbens, I. Chirupaddi, T.

III.197

532. Rhabdia, Mart.

1379. lycioides, Mart.

III.197

533. Tournefortia, L.

1380. argentea, L. f. Karan, S.

1381. WALKER A, Clarke.

III.198

III.198

534. Heliotropium, L.

1382. supinum, L. var. malabaricum, Retz.

1383. paniculatum, $\mathrm{Br}$.

III.199

1384. scabrum, Retz.

III.200

1385. indicum, L. Et-setiya, Et-honda, S'. Dimi-biya, Tedkodukku, T'.

III.200

III. 200

535. Trichodesma, Br'.

1386. indicum, Br. Kavil-tumpai, 'T.

1387. zeylanicum, Br.

III.201

III. 202

536. Cynoglossum, L.

1388. micranthum, Desf. Bu-katu-henda, S.

Forget-me-not.

III.203 var. $\beta$ decurrens, Moon.

90. Convolvulaceæ.

537. Erycibe, Roxb.

1389. paniculata, Roxb. Eta-miriya, Etcmbiriya, s. 
538. Rivea, Choisy.

1390. ornata, Choisy. Muchuddai, 'T.

III.205

539. Argyreia, Lour.

1391. tiliæfolia, Wight. Ma-banda, S.

III.206

1392. splendens, Sweet. III.207

1393. POPUlifolia, Choisy. Giri-tilla, S. III.207 var. $\beta$ coacta, Clarke.

1394. pomacea, Choisy. var. triflora, Clarke. III.208

1395. Choisyana, Wight.

III.208

540. Lettsomia, Roxb.

1396. aggregata, Roxb. var. osyrensis, Clarkc. III.209

1397. elliptica, Wight.

III. 209

1398. HANCORNIæFOLIA, Clarke.

III.210

541. Ipomæa, L.

*1399. digitata, L. Kiribadu, S.

III.212

*1400. hederacea, Jacq. Tali, T.

1401. dissecta, Willd.

*1402. Bona-nox, L. Alanga, Kalu-alanga, S.

Moon-flower.

III. 212

III.213

1403. grandiflora, Lam.

1404. JUCUNDA, Thw.

III. 213

III.214

1405. uniflora, Rœm. \& Scl. Potupala, S.

1406. pileata, Roxb.

1. LXVI.

1407. Wightii, Choisy.

III.215

III. 215

1408. bracteata, Wight.

III. 216

1409. Pes-tigridis, L. Divi-adiya, Divi-pahuru,

S.

III. 216

III.216

var. $\beta$ hepaticifolia, Clarke.

1410. eriocarpa, $\mathrm{Br}$.

III.217

1411. angustifolia, Jacq. Hin-madu, S.

III.217

1412. tridentata, Roth. Hawari-madu, S.

Mudiya-kuntal, T.

1413. reniformis, Choisy.

1414. chryseides, Ker. Kaha-tel-kola, S.

1415. staphylina, Røm. \& Scl.

III.218

III. 218

1416. eymosa, Rom. \& Sch. Kiri-madu, Maha-madu, S.

III. 219

III.219

1417. sepiaria, Kœnig. Rasa-tel-kola, S. Tali, T.

III.219

III. 220

var. $\beta$ stipulacea, Clarke.

1418. obscura, Ker. Tel-kola, S.

1419. campanulata, L.

1420. aquatica, Forsk. Kankun, S.

III. 220

III.221 var. $\beta$ parviflora, Trim.

1421. repens, Lam. Bin-tamburu, S.

III.221

III. 222 
1422. Turpethum, Br. Trastawalu, S. III.222

1423. denticulata, Choisy. III.223

1424. biloba, Forsk. Mudu-bin-tamburu, S. III.224

1425. vitifolia, Sweet.

III. 224

1426. palmata, Forsk.

III. 225

1427. dasysperma, Jacq.

III. 225

542. Hewittia, W. \& A.

1428. bicolor, W. \& A. Wal-trastawalu, S. III.226

543. Convolvulus, $\mathrm{L}$.

1429. parviflorus, Vahl.

III. 226

544. Evolvulus, L.

1430. alsinoides, L. Visnu-kranti, S. Vichnu-kiranti, T.

III.227

545. Breweria, Br.

1431. cordata, Bl.

III.227

546. Cressa, L.

1432. cretica, L. Panittanki, T.

III.228

547. Cuscuta, L.

1433. reflexa, Roxb.

III.22?

1434. chinensis, Lam. Aga-mula-neti-wel, S. III.22e

\section{Solanaceæ.}

548. Solanum, L.

1435. nigrum, L. Kalu-kan-weriya, S. Manaltakalli, T.

1436. læve, Dunal.

var. $\beta$ pubescens, Trim.

1437. pubescens, Willd.

III.231, Pl. LXVII.

1438. verbascifolium, L. Hekarilla, S.

III. 232

1439. giganteum, Jacq.

III. 232

1440. ferox, L. Malabatu, S.

III. 233

1441. torvum, Sw.

III. 233

1442. indicum, L. T'ibbatu, S.

III. 234

III.234

1443. xanthocarpum, Sclirad. \& Wendl. Elabatu, S. Vaddu, T. var. $\beta$ Jacquini, Thw. Katu-zvel-batu, S. Kandankattari, $\mathrm{T}$.

1444. trilobatum, I. Wal-tibbatu, S. Tutuvalai, $\mathrm{T}$.

III. 235

III. 236

549. Physalis, L.

1445. minima, L. Mottu, Hin-mottu, S.

III. 236

550. Withania, Pauq.

*1446. somnifera, Dun. Amukkara, S. Amukkirai, T. 
551. Datura, L.

1447. fastuosa, L. Attana, S. Venumattai, T.

III.238

92. Scrophulariaceæ.

552. Celsia, L.

1448. coromandeliana, Vahl.

III. 240

553. Adenosma, $\mathrm{Br}$.

1449. SUBREPENS, Benth.

1450. CAMPHORATUM, Hk. f. Kaha-gona-kola, S.

III.241

III.241

1451. capitatum, Benth. Nil-gona-kola, S. III.242

554. Limnophila, Br.

1452. conferta, Benth. Amba-wila, S.

1453. gratissima, $\mathrm{Bl}$.

III. 243

1454. hirsuta, Benth.

III. 243

1455. sessiliflora, Bl.

III. 244

1456. heterophylla, Benth.

III. 244

1457. racemosa, Benth.

III. 244

1458. gratioloides, $\mathrm{Br}$.

III. 245

III. 245

555. Herpestis, Grrtı. f.

1459. Monnieria, H. B. K. Lunu-wila, S. III. 246

1460. floribunda, $\mathrm{Br}$.

III. 246

556. Dopatrium, Hamilt.

1461. nudicaule, Ham.

III. 247

1462. lobelioides, Benth.

III. 247

1463. junceum, Ham. Bin-savan, S.

III. 247

557. Artanema, Don.

1464. sesamoides, Benth. Gas-kotala, S.

III. 248

558. Torenia, L.

1465. asiatica, L. Kotala-wel, S.

III. 249

1466. hirtella, Hk. f.

III. 249

559. Vandellia, L.

1467. crustacea, Benth.

1468. hirsuta, Ham.

III. 250

1469. scabra, Benth.

III. 250

1470. pedunculata, Benth.

III.251

1471. angustifolia, Benth.

III.251

III.251

560. Ilysanthes, Rafin.

1472. hyssopioides, Benth.

III. 252

1473. rotundifolia, Benth. 
561. Bonnaya, Link \& Otto.

III. 253

1474. brachiata, Link \& Otto.

III. 253

1475. veronicæfolia, Spreng. Wila, S.

III. 253

1476. tenuifolia, Spreng.

III. 254

562. Microcarpæa, $\mathrm{Br}$.

1477. muscosa, Br.

III.254

563. Peplidium, Del.

1478. humifusum, Del.

III.255

564. Striga, Lour.

1479. orobanchoides, Benth.

1480. lutea, Lour.

III. 255

1481. euphrasioides, Benth.

III. 256

III.256

565. Sopubia, Ham.

1482. delphinifolia, G. Don.

III. 257

1483. trifida, Ham.

III.257

566. Centranthera, Br.

1484. procumbens, Benth. Dutu-satutu, S III.258, Pl. LXVIII.

1485. hispida, Br.

III. 259

1486. humifusa, Wall.

III.259

567. Pedicularis, L.

1487. zeylanica, Benth.

III.260

93. Orobanchaceæ.

568. Aginetia, L.

1488. indica, L. Kolikara-mal, S.

III.261

1489. pedunculata, Wall.

III.261

569. Christisonia, Gardn.

1490. subacaulis, Gardu.

1491. ThWaitesir, Trim.

1492. TRICOLOR, Gardn.

III.262

III.263, Pl. LXIX.

III. 263

var. $\beta$ grandiflora, Hk. f.

1493. bicolor, Gardn.

III.264

var. $\beta$ pallidiflora, Thw.

var. $\gamma$ speetabilis, Trim.

1494. ALBIDA, Thw.

III.265

570. Campbellia, Wight.

1495. cytinoides, Wight.

III.265

94. Lentibulariaceæ.

571. Utricularia, L.

1496. stellaris, L. f.

1497. flexuosa, Vahl. Diya-pasi, S.

III.267

1498. exoleta, Br.

III. 267

III.268

$6(11) 10$ 


\section{$(64)$}

1499. cærulea, L. Nil-monaressa, S.

III. 268

1500. affinis, Wight.

III. 269

1501. reticulata, Smith. Nil-monaressa, S.

III. 269 var. $\beta$ stricticaulis, Kœnig.

1502. capillacea, Wall.

1503. bifida, $L$.

1504. nivea, Vahl. var. $\beta$ rosea, Thw.

1505. orbiculata, Wall.

III. 270

III. 270

III. 270

III.271

\section{Gesneraceæ.}

572. Eschynanthus, Jack.

1506. zeylanica, Gardn. var. $\beta$ pinguis, Clarke.

III. 272

573. Didymocarpus, Wall.

1507. humboldtiands, Gardn. var. $\beta$ primulæfolius, Trim. var. $\gamma$ recedens, Clarke.

1508. FLoccosus, Thw.

1509. ZEYLANICUS, Br.

574. Chirita, Ham.

1510. MOONII, Gardn.

1511. WALKeri, Gardn. var. $\beta$ parviflora, Clarke.

1512. ZEYLANICA, Hook.

III. 275 III. 275

III. 276 var. $\beta$ angusta, Clarke.

575. Championia, Gardn.

1513. Reticulata, Gardn.

III. 277

576. Klugia, Schlecht.

1514. Notoniana, A. DC. Diya-nilla, S.

III. 277 var. $\beta$ glabra, Clarke.

1515. ZEYLANICA, Gardn.

III. 278

577. Epithema, Bl.

1516. carnosum, Benth. var. zeylanicum, Clarke.

III. 279

578. Isanthera, Nees.

1517. permcllis, Nees.

III. 280

96. Bignoniaceæ.

579. Oroxylum, Vent.

1518. indicum, Vent. Totila, S.

III.281

580. Dolichandrone, Seem.

1519. Rheedii, Seem. Diya-danga, S. Vilpadri, T. 
581. Stereospermum, Cham.

1520. chelonioides, DC. Lunu-madala, Dunumadala, S. Padri, T.

III. 283

97. Pedaliace $æ$.

582. Pedalium, L.

1521. Murex, L. Et-nerenchi, S. Peru-nerinchi, Anai-nerinchi, T.

III.285

583. Sesamum, L.

*1522. indicum, L. Tel-tala, S. Ella, T. Gingili, Gingelly.

III.285

98. Acanthaceæ.

584. Thunbergia, L. f.

1523. fragrans, Roxl).

III.28s var. $\beta$ vestita, Nees. var. $\gamma$ parviflora, Trim.

585. Elytraria, Vahl.

1524. crenata, Vahl. var. $\beta$ lyrata, Valil.

III.289

586. Ebermaiera, Nees.

1525. zeylanica, Nees.

III.290

587. Cardanthera, Ham.

1526. uliginosa, Ham.

III.291

1527. balsamica, Clarke.

III.291

1528. verticillata, Clarke.

III.291

1529. Thwaitesir, Benth.

III.292

588. Hygrophila, $\mathrm{Br}$.

1530. salicifolia, Nees.

III.293

153I. quadrivalvis, Nces.

III. 293

1532. spinosa, And. Katu-ikiri, S. Nirmulli, $\mathrm{T}$.

III.293

589. Calophanes, D. Don.

1533. Nagchana, Nees. Paduvan, 'T.

1534. littoralis, And. Paraddai, T.

III. 294

III. 295

590. Ruellia, L.

1535. ringens, L. Nil-puruk, S.

III. 295

1536. patula, Jacq.

III.296

591. Phaylopsis, Willd.

1537. parviflora, Willd.

III. 296

592. Drdalacanthus, T. And.

1538. montanus, And.

III. 297 
593. Stenosiphonium, Nees.

1539. Russellianum, Nees. Bu-nelu, S. Nelu, $\mathrm{T}$.

III. 298

var. $\beta$ subsericeum (Nees.), Trim.

594. Strobilanthes, Bl. Nelu, S.

1540. viscosus, And.

III.301

- var. $\beta$ digitalis, Clarke.

var. $\gamma$ argutus, Clarke.

1541. NOCKII, Trim.

1542. STENODON, Clarke.

1543. exAREOLATUS, Clarke.

1544. RHYTISPERMUS, Clarke.

1545. NIGRESCENS, And.

1546. RHAMNIFOLIUS, And.

1547. Deflexus, And.

1548. LaNCEOLATUS, Hook.

1549. Walkeri, Arn. var. $\beta$ stenocarpa, Clarke.

1550. THWaITESII, And.

III.301, Pl. LXX.

III.302

III.303

III. 303

III.303

III.304

III.304

III. 305

III.305

1551. caudatus, And.

III.306

var. $\beta$ laniceps, Clarke.

1552. anceps, Nees.

III.306

1553. PUNCTATUS, Nees.

III.307

1554. aRNotTitan US, Nees.

III.307

1555. ASPERRIMUS, Nees.

III.308

1556. TRIFIDUS, Nees.

III. 308

1557. exsertus, Clarke. var. $\beta$ integra, Clarke.

1558. GardNerianus, And.

1559. vestitus, Nees.

III.309

III.309

1560. HOOKERI, Nees.

1561. CALYCINUS, Nees.

1562. LAXUS, And.

1563. ZEYlaniCUS, And.

1564. sexennis, Nees. var. $\beta$ argutus, Clarke. var. $\gamma$ hirsutissimus, And.

1565. HELICOIDES, And.

III. 310 III.310

III.311

III.311

III.312

III.312

III.313

1566. Paniculatus, And.

1567. PUlcherrimus, And.

III.314

III.314

III.315

595. Blepharis, Juss.

1568. bœrhaaviæfolia, Pers.

III.316

1569. molluginifolia, Pers.

III.316

596. Acanthus, L.

1570. ilicifolius, L. Ikili, Katu-ikili, S. var. $\beta$ integrifolius, And.

III.317 


\section{$(67)$}

597. Barleria, L.

1571. Prionitis, L. Katu-karandu, S.

1572. mysorensis, Roth. Katu-nelu, S. Kirimulla, Kikkiri, Ikkiri, T.

1573. noctiflora, L. f.

1574. involucrata, Nees.

1575. vestita, And.

1576. Arnottiana, Nees. var. $\beta$ glabra, Trim.

1577. NutaNs, Nees.

III. 318

1578. nitida, Nees.

III.319

III.319

III. 320

III. 320

III.321, Pl. LXXI.

III.321

III.322

598. Crossandra, Salisb.

1579. undulæfolia, Salisb.

III.322

var. $\beta$ crocea, Trim.

var. $\gamma$ axillaris (Nees.), Trim.

599. Asystasia, Bl.

1580. coromandeliana, Nees. Puruk, S. Peyppatchotti, T.

1581. chelonioides, Nees.

1582. variabilis, Trim.

III. 323

III. 324

III.324

600. Eranthemum, L.

1583. malabaricum, Clarke.

III.325

601. Andrographis, Wall.

1584. paniculata, Nees. Hin-bin-kohomba, S. Nila-vempu, T.

III.326 var. $\beta$ glandulosa, Trim.

1585. macrobotrys, Nees.

JII.327 var. $\beta$ parvifolia, Clarke.

1586. alata, Nees.

III.327

1587. echioides, Nees. Hakan, S.

III.327

602. Gymnostachyum, Nees.

1588. zeYlanicum, Arn. and Nees.

III. 328

1589. Thwattesir, And.

1590. Paniculatum, And.

1591. Sanguinolentum, And.

III. 329

III. 329

III. 330

1592. HiRsutum, And.

III. 330

603. Lepidagathis, Willd.

1593. hyalina, Nees. var. lophostachyoides, Nees.

1594. ZeYLANICA, Nees.

III.331

1595. WALKERIANa, Nees.

III. 331

1596. fasciculata, Nees.

III.332

III.332

604. Monotheeium, Hoehst.

1597. aristatum, And. 
605. Justicia, L.

1598. Betonica, L. Sudu-puruk, S.

III. 334

1599. ZEYLANICA, And.

III.334 var. $\beta$ capitata, And.

1600. tranquebarensis, L. $f$.

1601. Gendarussa, Burm. f. Kalu-waraniya, S. Kavunochchi, T.

1602. HOOKERIANa, And.

1603. glabra, Kœn.

III.335

1604. procumbens, L. Mayani, S. var. $\beta$ latispica, Clarke.

1605. ROYENIANA, Clarke.

1606. diffusa, Willd.

III.335

III. 336

III. 336

III. 337 var. $\beta$ prostrata, Roxb.

III.337

III. 338

606. Adhatoda, Nees.

160\%. Vasica, Nees. Agaladara, Wanepala, S. Adatodai, Pavettai, T. Malabar nut.

III.338

607. Rhinacanthus, Nees.

1608. communis, Nees. Anitta, S. Nagamulli, $\mathrm{T}$.

III. 339

608. Ptyssiglottis, And.

1609. RADICOSA, And.

III.340

609. Ecbolium, Medik.

1610. Linneanum, Kurz.

III.341

610. Rungia, Nees.

1611. latior, Nees.

1612. parviflora, Nees. var. $\beta$ pectinata, Clarke.

III.342

III.342

1613. repens, Nees. Sulu-nayi, S. III.343

1614. apiculata, Bedd.

III.343

611. Dicliptera, Juss.

1615. zeylanica, Nees. var. $\beta$ Neesii, Trim.

III.344

99. Verbenaceæ.

612. Lantana, L.

1616. indica, Roxb.

III.346

613. Lippia, L.

1617. nodiflora, Rich. Heri-mena-detta, S. Podutalai, T.

614. Bouchea, Cham.

1618. hyderabadensis, Walp. 
615. Stachytarpheta, Vahl.

*1619. indica, Vahl. Bala-nakuta, S. Naioringi, $\mathrm{T}$.

III.348

var. $\beta$ jamaicensis, Trim.

616. Priva, Adans.

1620. leptostachya, Juss.

III.349

617. Callicarpa, L.

1621. lanata, L. Illa, S.

III.350

618. Premna, L.

1622. PURPURASCENS, Thw.

III.351, Pl. LXXII.

1623. corymbosa, Rottl.

1624. serratifolia, L. Midi, S. Erumaimullai, $\mathrm{T}$.

1625. tomentosa, Willd. Bu-seru, S. Kolukkutti, T.

1626. THWAITESII, Clarke.

1627. latifolia, Roxb. Maha-midi, S. Pachumullai, T.

*1628. procumbens, Moon. Mullai, T.

III.351

III.352

III.352

III.353

III.353

II1.354

619. Gmelina, L.

1629. arborea, Roxb. Et-demata, S.

1630. asiatica, I. Demata, S. Kumil, T.

III.355

III.355

620. Vitex, L.

1631. trifolia, L. Nochchi, T.

1632. Negundo, L. Nika, Nil-nika, Sudu-nika,

S. Vennochchi, T.

1633. altissima, L. f. Milla, Miyan-milla, Sapu-milla, S. Kadamanakku, T.

var. $\beta$ zeylanica, Clarke.

III.356

III.357 var. $\gamma$ alata, Trim.

1634. Leucoxylon, L. f. Nebedda, S. Kaddunochchi, Nir-nochchi, T.

III.358

621. Clerodendron, L.

1635. inerme, Gærtn. Wal-gurenda, S. Pinchil, Pinari, T.

1636. Phlomidis, L. f. Vatamadakki, T.

1637. serratum, Spreng. Ken-henda, S. Vatamadakki, $\mathrm{T}$.

1638. infortunatum, L. Gas-pinna, S.

III.359

III.360

III.357

III. 360

III.361

622. Glossocarya, Wall.

1639. SCANDENS, Trim.

III.362, Pl. LXXIII.

623. Symphorema, Roxb.

1640. involueratum, Roxb.

III.363 
624. Avicennia, L.

1641. officinalis, L. Kanna, T. White mangrove.

III.363

100. Labiatæ.

625. Ocimum, L.

III.365

1642. canum, Sims. Hin-tala, S. Kanchankorai, $\mathrm{T}$.

1643. sanctum, L. Maduru-tala. S.

III.365

III.366

1644. adscendens, Willd.

III.366

*1645. gratissimum, L. Gas-tala, Otala, S. var. $\beta$ suave, Hk. f.

626. Geniosporum, Wall.
1646. clongatum,
1647. prostratum,
var. $\beta$ gracil
627. Moschosma, Rchb.

626. Geniosporum, Wall.
1646. clongatum, Benth.
1647. prostratum, Benth.
var. $\beta$ gracile, Thw
627. Moschosma, Rchb.

626. Geniosporum, Wall.
1646. clongatum, Benth.
1647. prostratum, Benth.
var. $\beta$ gracile, Thw
627. Moschosma, Rehb. var. $\beta$ gracile, Thw.

III.367

1648. polystachyum, Benth.

III. 368

III.368

627. Moschosma, Rchb.

628. Orthosiphon, Benth.

1649. glabratus, Benth.

III .369

III. 369

629. Plectranthus, L'Herit.

1650. NIGRESCENS, Benth.

1651. Walkeri, Arn.

III. 370

III.371

1652. GARDNERI, Thw.

III.371

1653. coleoides, Benth.

III.372

1654. CAPILLIPES, Benth.

III. 372

1655. menthoides, Benth.

III.372

630. Coleus, Lour.

1656. barbatus, Benth. Wal-kapura-walliya,

$\mathrm{S}$.

1657. malabaricus, Benth.

III.373

var. $\beta$ leptostachys, Hk. f.

III.374

1658. INFLatus, Benth.

1659. ELONGatus, Trim.

III.375

III.375, P1. LXXIV.

631. Anisochilus, Wall.

1660. carnosus, Wall. Gal-kapura-walliya, S. III.376 1661. paniculatus, Benth. III.377, Pl. LXXV. 1662. velutinus, Trim. Bolvila, Bolila, S. III.377

632. Pogostemon, Desf.

1663. Heyneanus, Benth. Gan-kollan-kola, S. III.378

1664. RUPESTRIS, Benth.

1665. HIRSUTUs, Benth.

III.379

1666. Reflexus. Benth.

III. 379

III. 379 
fi33. Dysophylla, Bl.

1667. auricularia, Bl. Hemanilla, S.

1668. verticillata, Benth.

III. 380

III.380

634. Mentha, L.

1669. javanica, Bl. Odu-talan, S.

III.381

635. Calamintha, Mœnch.

1670. umbrosa, Benth.

III.381

636. Scutellaria, L.

1671. violacea, Heyne. var. $\beta$ glabra, Trim.

III.382

1672. robusta, Benth.

III.383

1673. oblonga, Bently.

III.383

637. Anisomeles, $\mathrm{Br}$.

1674. ovata, $\mathrm{Br} . \quad Y a k$-wanassa, S.

III.384

1675. malabarica, Br. Pey-maruddi, T.

III.384

638. Leucas, Br.

1676. mollissima, Wall.

III.385

1677. marrubioides, Desf. Sudu-tumba, S. III.385

1678. angularis, Benth.

III.385

1679. bifora, Br. Geta-tumba, S. Peyt-tumpai,

$\mathrm{T}$.

III. 386

1680. longifolia, Benth.

1681. zeylanica, Br. Geta-tumba, Mudi-tumpai, $\mathrm{T}$.

III.386

III.387 var. $\beta$ Walkeri, Hk. f.

639. Leonotis, $\mathrm{Br}$.

1682. nepetæfclia, Br. Maha-yak-wanassa, S.

Kasitumpai, T.

III.387

640. Teucrium, L.

1683. tomentosum, Heyne.

III.388

101. Plantaginaceæ.

641. Plantago, L.

1684. major, L. var. asiatica, Dene.

III.389

\section{INCOMPLETAE.}

102. Nyctaginaceæ.

642. Boerhaavia, L.

1685. diffusa, L. Pita-sudu-pala, S. Mukkaraichchi, Karichcharanai, T.

III.390

1686. repanda, Willd.

III.390

643. Pisonia, L.

1687. aculeata, L.

III.391

$6(11) 10$ 
103. Amarantaceæ.

644. Celosia, L.

1688. argentea, L. Kiri-henda, S.

III.393

1689. pulchella, Moq.

III.393

1690. polygonoides, Retz.

III. 394

645. Allinania, $\mathrm{Br}$.

1691. nodiflora, Br. Kumatiya, S.

III.394 var. $\beta$ longepedunculata, Trim.

646. Digera, Forsk.

1692. arvensis, Forsk. Toggil, T.

III.395

647. Amarantus, L.

1693. spinosus, I. Katu-tampala, S. Mudkirai, T.

III.396

*1694. gangeticus, L. Sudu-tampala, S. Chirukirai, Araikkirai, T.

III.396

1695. mangostanus, $\mathrm{L}$.

III.397

1696. viridis, L. Kura-tampala, S.

III. 397

1697. polygonoides, L. Araikkirai, T. III.397

648. Cyathula, Lour.

1698. ZEYLANICA, Hk. f.

III.398

1699. prostrata, Bl. Bin-karal-heba, S.

III.398

649. Pupalia, Juss.

1700. atropurpurea, Moq. Wel-karal-heba, S. III.399

1701. orbiculata, Wight. Kumiddil, Pichukodiya, T.

III. 400

650. Psilotrichum, Bl.

1702. SClERANTHUM, Thw.

1703. calceolatum, Moq.

III. 400

III. 400

651. Nothosærua, Wight.

1704. brachiata, Wight. Tampala, S. Chirupilai, $\mathrm{T}$.

III.401

652. Arua, Forsk.

1705. javanica, Juss.

III.402

1706. lanata, Juss. Pol-kudu-pala, S.

III.402

1707. Monsonire, Mart.

III. 403

653. Achyrantlies, L.

1708. aquatica, $\mathrm{Br}$.

1709. aspera, L. Gas-karal-heba, S. Nayuruvi,

T.

var. $\beta$ argentea, Hk. f.

1710. bidentata, $\mathrm{Bl}$.

III.403

III.404

III. 404

1711. DIANDRA, Roxb.

III.405 
654. Alternanthera, Forsk.

1712. triandra, Lam. Muknnu-wenna, S. Ponnankani, T.

III.405

104. Chenopodiaceæ.

655. Atriplex, I.

1713. repens, Roth. Elichchevi, T. III.406

656. Arthrocnemum, Moq.

1714. indicum, Moq. Kotanai, T.

III.407

657. Salicornia, I.

1715. brachiata, Roxb.

III.408

658. Suæda, Forsk.

1716. monoica, Forsk.

III.408

1717. maritima, Dumort.

III. 409

1718. nudiflora, Moq. Umiri, T.

III.409

659. Basella, $\mathrm{L}$.

1719. rubra, L. Niviti, S. Pasalai, T.

III.410

105. Polygonaceæ.

660. Polygonum, L.

1720. tomentosum, Willd. Sudu-kimbulwenna, S.

1721. glabrum, Willd.

III.411

III.412

1722. minus, Huds.

1723. barbatum, L. Ratu-kimbulwenna, S.

III.412

1724. serrulatum, Lagasca.

III. 412

1725. punctatum, Ham.

1726. chinense, L.

1727. strigosum, $\mathrm{Br}$.

1728. prætermissum, Hk. f.

1729. pedunculare, Wall.

III. 413

III. 413

III.413

III.414

III.414

III.415

106. Podostemaceæ. ${ }^{1}$

661. Lawia, Griff.

1730. zeylanica, Tul.

III.416

var. $\beta$ Gardneriana, Willis.

var. $\gamma$ Parkiniana, Willis.

662. Dicrea (Du Pet Th.), Tul.

1731. ELONGATA, Tul. (Podostemon elongatus, Gardn.)

1732. stylosa, Wight.

III. 417

III. 417

var. $\alpha$ fucoides, Willis. (I'. algaformis, Trim.)

var. $\beta$ laciniata, Willis.

1 See Willis, A Revision of the Podost. of India and Ceylon, Ann. Perad., I., p. 181. 1902. 
663. Podostemon, Tul.

1733. subulatus, Gardn. var. mavoeliæ, Willis. III.418

664. Hydrobryum. Endl.

1734. olivaceum, Tul. var. zeylanicum, Willis. III.418

1735. lichenoides, Kurz. var. kelense, Willis.

665. Farmeria, Willis.

1736. METZGERIOIDES, Willis. III.419, Pl. LXXVI.

107. Nepenthaceæ.

666. Nepenthes, L.

1737. DistiLlatoria, L. Bandura-wel, S.

Pitcher-plant.

III.420

108. Aristolochiaceæ.

667. Bragantia, Lour.

1738. Wallichii, Br.

[II.42]

var. $\beta$ latifolia, Duchart.

var. $\gamma$ brachycarpa, Hk. $f$.

668. Aristolochia, L.

1739. bracteata, Retz. Adutin-tappalai, 'T.

III.422

1740. indica, L. Sap-sanda, S. Perumaruntu, $\mathrm{T}$.

109. Piperaceæ.

669. Piper, L.

*1741. longum; L. Tippili, S. and T. Long pepper.

III.424

*1742. Betle. L. Bulat, Bulat-wel, S. Vettilai,

T. Betel pepper.

III. 425 var. $\beta$ Siriboa, Trim. Rata-bulat-wel, Siri-bo, $\mathrm{S}$.

1743. THWAITESI, Cas. DC. III.426, Pl. LXXVII. 1744. nigrum, L. Miris, Gam-miris-vel, S.

Milaku, T. Black pepper.

1745. ZEYLANICUM, Miq.

1746. TRINEURON, Miq.

.427

III. 427 var. $\beta$ laxiflorum, Trim.

1747. argyrophyllum, Miq. Wal-gam-miriswel, S.

III.428

var. $\beta$ Walkeri, Trim.

III.428

1748. sylvestre, Lam. Wal-gam-miris-wel, Mala-miris-wel, S.

1749. subpeltatum, Willd. Mala-labu, S. 
670. Peperomia, Ruiz. \& Pav.

1750. PSEUDO-RHOMBEA, Cas. DC.

III.430 var. $\beta$ tenuis, Trim.

1751. Wightiana, Miq.

III.431 var. $\beta$ Ritigalensis, Willis.

1752. CONFUSA, Hk. f.

III.431

1753. dindigulensis, Miq.

III.431 var. $\beta$ hirsuta, Trim.

1754. reflexa, A. Dietr.

III.432

110. Chloranthaceæ.

671. Chloranthus, Swartz.

1755. brachystachys, B!.

III.433

111. Myristicaceæ.

672. Myristica, L.

1756. Laurifolia, Hk. f. \& Th. Malaboda, S. Palmanikam, T.

1757. ZEYLANICA, A. DC.

III.434

1758. HORSFIELDIA, Bl. Ruk, S.

III.434

1759. Irya, Gærtn. Iriya, S.

III.435

III.435

\section{Monimiaceæ.}

673. Hortonia, Wight.

1760. FLORIBUNDA, Wight. Wawiya, S.

III.436 var. $\beta$ ovalifolia, Hk. f. \& Th.

1761. andUstifolia, Trim. III.437, PJ. LXXVIII.

\section{Lauraceæ.}

674. Cryptocarya, $\mathrm{Br}$

1762. Wightiana, Thw. Gal-mora, S.

III. 439

1763. MembranaCeA, Thw. Tawenna, S.

III. 439

675. Beilschmiedia, Nees.

1764. ZEYLANICA, Trim.

III.440

676. Cinnamomum, Bl.

1765. zeylanicum, Bl. Kurundu, S. Karuva,

T. Cinnamon.

III.440

1766. MULTIFLORUM, Wight. Wal-kurundu, S. III.441

1767. OVALIFOLIUM, Wight.

III.442

1768. LITSE. FOLIUM, Thw. $K u d u-k u r u n d u$, S. III.442

1769. CITRIodorum, Thw. Pengiri-kurundu, S. III.443

677. Machilus, Nees.

1770. macrantha, Nees. Ululu, S.

III.443

678. Alseodaphne, Nees.

1771. semecarpifolia, Nees. Wewarani, S.

Ranai, Yavaranai, T. 
679. Actinodaphne, Nees.

1772. MOLOCHINA, Nees. var. $\beta$ Moonii, Hk. $f$.

1773. STENOPHYLLA, Thw.

III.445

1774. ELEGANS, Thw.

III. 446

1775. GLAUCA, Nees.

1776. PISIFERA, Hk. f.

III. 446

III.446

1777. AMBIGUA, Hk. f.

III. 447

var. $\beta$ orbicularis, Trim.

1778. SPECIOSA, Nees. Elephants' ears. III.448

680. Litsea, Lam. var. $\beta$ Candolleana, Hk. $f$.

1779. tomentosa, Heyne. Kosbada, Landittan, S.

III. 449

1780. chinensis, Lam. Bomi, Bombi, S. Elumpurukki, T.

III. 449

1781. UNDULATa, Hk. f.

1782. CAULIFLORA. Trim. Rat-keliya, S.

III. 450

1783. hookeriana, Meissn.

III. 450

1784. Nemoralis, Thw.

1785. Ovalifolia, Thw.

III.451

1786. GLABERRIMA, Thw.

III.451

III.451

1787. ITEODAPHNE, Thw.

III. 452

var. $\beta$ angustata, Meissn.

1788. GARDNERI, Thw.

III.452

1789. Fuscata, Thw.

1790. zeylanica, Nees. Dawul-kurundu,

III.453

III. 453

Kududawula, S. Wild cinnamon. var. $\beta$ rubrinervia, Meissn.

681. Lindera, Thunb.

1791. LaNCIFolia, Thw.

III.454

682. Cassytha, L.

1792. filiformis, L.

III.455

1793. capillaris, Meissn.

III.455

683. Hernandia, L.

1794. peltata, Meissn. Palatu, S.

III.456

114. Proteaceæ.

684. Helicia, Lour.

1795. ZEYLANICA, Gardn.

III.457, Pl. LXXIX.

115. Thymelæaceæ.

685. Wikstrœmia, Endl.

1796. canescens, Meissn. 
686. Lasiosiphon, Fresen.

1797. eriocephalus, Dene. Naha, S.

III.459 var. $\beta$ zeylanicus, Meissn.

687. Phaleria, Jack.

1798. CAULIFlora, Bedd.

III.459

688. Gyrinops, Gærtn.

1799. WALLa, Gæitn. Walla, Patta-walla, S. III.460

116. Elæagnaceæ.

689. Elæagnus, L.

1800. latifolia, L. Wel-embilla, Katu-embilla, S. III.461 var. $\beta$ Thwaitesii, Trim.

\section{Loranthaceæ.}

690. Loranthus, L. Pilila, S. Kuruvichchai, T.

1801. NODIFLORUS, Thw.

III.463

1802. MAB

III.463

1803. ENSIFOLIUS, Thw.

III.464

1804. Hookerianus, W. \& A.

III. 464

1805. Scuriula, L.

III. 465

1806. cordifolius, Wall.

III.465

1807. tomentosus, Heyne.

III.465 var. $\beta$ incanus, Trim.

1808. cuneatus, Heyne.

1809. SCLEROPHYLLUS, Thw.

III. 466

1810. Ligulatus, Thw.

III.466

1811. SUBORBICULARIS, Thw.

III. 467

1812. longiflorus, Desrouss.

III. 467

var. $\beta$ amplexifolius, Thw.

1813. LONCHIPHYLLUS, Thw.

1814. neelgherrensis, W. \& A.

III. 468

1815. GARDNERI, Thw.

III. 468

1816. loniceroides, $\mathrm{L}$.

IIJ.468

III. 469

1817. capitellatus, W. \& Á.

III. 469

III. 470

691. Viscum, L. Pilila, S. Kuruvichchai, T.

1818. orientale, Willd.

1819. monoicum, Roxb.

III.471

1820. capitellatum, Sm.

III.471

1821. ramosissimum, Wall.

III. 471

1822. articulatum, Burm.

III. 472

1823. japonicum, Thunb.

III.472

III. 472

692. Notothixos, Oliv.

1824. FLOccosus, Oliv.

III.473, Pl. LXXX.

693. Ginalloa, Korth.

1825. Spathulivolia, Oliv. 
118. Santalaceæ.

694. Osyris, $\mathrm{L}$.

1826. arborea, Wall.

III.474

695. Scleropyrum, Arn.

1827. Wallichianum, Arn.

III. 475

119. Balanophoraceæ.

696. Balanophora, Forct.

1828. indica, Wall.

III. 476

1829. THWATTESII, Eichl.

III.477, Pl. LXXXI.

120. Euphorbiaceæ.

ij97. Euphorbia, L.

1830. Antiquorum, L. Daluk, S. Chaturakalli, T.

IV.4

1831. tortilis, Rottl. Sinuk, S.

IV.5

1832. Atoto, Forst.

IV.6

1833. rosea, Retz. Mudu-dada-kiriya, S.

IV. 6

1834. cristata, Heyne.

IV.6

1835. hypericifolia, L. Ela-dada-kiriya, S.

IV.7

1836. hirta, L. Bu-dada-kiriya, S. Palavi, T. IV.7

1837. thymifolia, L. Bin-dada-kiriya, S. Chittirapalavi, $\mathbf{T}$.

1838. Rothiana, Spreng.

698. Sarcococca, Lindl.

1839. pruniformis, Lindl. var. $\beta$ zeylanica, Hk. $f$.

IV.9 var. $\gamma$ brevifolia, Muell. Arg.

699. Bridelia, Willd.

1840. retusa, Spreng. Keta-kala, S. Mulvenkai, $\mathrm{T}$.

1841. MoonII, Thw. Pat-kala, S.

1842. scandens, Willd.

IV.10

IV.11

[V.11

700. Cleistanthus, Hk. f.

1843. collinus, Benth. Madara, S.

IV.12

1844. асUMinatus, Muell. Arg.

IV.12

1845. Robustus, Muell. Arg.

IV.13

1846. patulus, Muell. Arg.

IV.13

1847. Pallidus, Muell. Arg. Visa, $\mathrm{T}$.

IV.13

var. $\beta$ subglauca, Trim.

1848. Ferrugineus, Muell. Arg.

IV.14

701. Actephila, Bl.

1849. neilgherrensis, Wight.

IV.14

702. Agyneia, Vent.

1850. bacciformis, A. Juss. Et-pitawakka, S. IV.15 
703. Sauropus, BI.

1851. albicans, Bl. Mella-dum-kola, S. IV.16

1852. RE'ROVERSUS, Wight. IV.16

1853. ASSIMILIS, Thw. IV.17

1854. RIGIDUS, Thw. IV.17

704. Phyllanthus, L.

1855. Thwattesianus, Muell. Arg. IV.18

1856. reticulatus, Poir. Wel-kayila, S. Pula, Pullant, Mipullanti, T.

1857. Emblica, L. Nelli, S. Topu-nelli, T. IV.19

1858. polyphyllus, Willd. IV.20

1859. maderaspatensis, $\mathrm{L}$. IV.20

1860. Rheedii, Wight. IV.21

1861. Urinaria, L. Rat-pitawakka, S. IV.21

1862. MYRTIFolius, Moon. IV.22

1863. simplex, Retz. IV.22

var. $\beta$ Gardnerianus, Muell. Arg.

1864. Niruri, L. Pitawakka, S. Kilkaynelli, T.IV.23

1865. rotundifolius, Klein. IV.23

1866. Batllonianus, Muell. Arg. IV.23

1867. anabaptizatus, Muell. Arg. IV.24

1868. OREOPHILUS, Muell, Arg. IV.24

1869. longiflorus, Heyne. IV.25

1870. HaKgalensis, Thw. IV.25

1871. CINereus, Muell. Arg. IV.26

1872. AFFinis, Muell. Arg. IV.26

1873. indicus, Muell. Arg. Karawu, S. IV.27

1874. CYaNOSPERMUS, Muell.Arg. Sudu-liyan, Kulu-liyan, S.

IV.27

705. Glochidion, Forst.

1875. zeylanicum, A.Juss. Hunu-kirilla, S. IV.28 var. $\beta$ tomentosum, Trim.

1876. BRACHYLOBUM, Muell. Arg. IV.29

1877. PYCNOCARPUM, Bedd.

var. $\beta$ elliptica, Hk. f.

1878. RIGIDUM, Muell. Arg.

IV.29

1879. sp. nov. (Ritigala). ${ }^{1}$

IV.30

1880. CORIACEUM, Thw.

1881. Nemorale, Thw.

IV.30

IV.31

1882. GARDNERI, Thw.

var. $\beta$ acuminata, Trim.

IV.31

1883. MONTANUM, Thw.

IV.31

1884. MOONI, Thw. Bu-hunu-kirilla, S.

IV.32

I Willis, 'The Flora of Ritigala, Aun. Perad, ITI., p. 285, 1906.

$6(11) 10$ 
706. Flueggea, Willd.

1885. leucopyrus, Willd. Hin-katu-pila, s. Mudpulanti, $\mathrm{T}$.

IV.33

707. Breynia, Forst.

1886. patens, Hk. f. Wal-murunga, S.

IV.33

1887. rhamnoides, Muell. Arg. Gas-kayila, S. Manipulnati, $\mathrm{T}$.

IV .34

708. Putranjiva, Wall.

1888. Roxburghii, Wall. Vitchurunai, Karippalai, $\mathrm{T}$.

IV.35

1889. zeYlanica, Muell. Arg. Pelan, S. IV.35

709. Hemicyclia, W. \& A.

1890. sepiaria, W. \& A. Wira, S. Virai, T. IV. 36

1891. LANCEOLATA, Thw.

IV.37

1892. GARDNERI, Thw. Gal-wira, Eta-wira, S. IV.37,

710. Cyclostemon, Bl.

Pl. LXXXII.

1893. macrophyllus, $\mathrm{Bl}$.

IV.38

711. Mischodon, Thw.

1894. zeylanicus, Thw. Tammanna, S. Tampanai, $\mathrm{T}$.

IV.38

712. Aporosa, B1.

1895. LAtifolia, Thw. Mapat-kebella, Kampotta, Pepiliya, S.

IV .39

1896. Lindleyana, Baill. Kebella, Barawaembilla, S.

1897. Lanceolata, Thw. Hin-kebella, S.

1898. acuminata, Thw.

$1 \mathrm{~T} .40$

1899. FUSIFORMIS, Thw.

IV.40

IV.41

IV.41

713. Daphniphyllum, Bl.

1900. glaucescens, Bl. IV.42

714. Antidesma, L.

1901. Ghæsembilla, Gærtn. Bu-embilla, S. IV.43

1902. Bunius, Spreng. Karawala-kebella, S. IV.43 var. $\beta$ Thwaitesianum, Trim.

1903. zeylanicum, Lam. Hin-embilla, S. IV.44

1904. diandrum, Roth.

IV.44

1905. PYRIFOLIUM, Muell. Arg.

IV.45

715. Jatropha, L.

*1906. glandulifera, Roxb. Alalai, T.

IV.45

716. Croton, L.

1907. reticulatus, Heyne.

1908. oblongifolius, Roxb. Milla-kunari, T. 


\section{$(81)$}

1909. aromaticus, L. Wel-keppiliya, S. Teppaddi, T.

var. $\beta$ lacciferus, Trim. Keppitiya,

Gas-keppiliya, S.

1910. caudatus, Geisel.

IV.48

1911. MOONII, Thw.

IV. 49

1912. Klotzschianus, Thw.

IV. 49

1913. NIGRO-VIRIDIS, Thw.

IV.49

717. Givotia, Griff.

1914. rottleriformis, Griff. Puttalai, T.

IV.50

718. Trigonostemon, Bl.

1915. DIPLOPETALUS, Thw. IV.51, Pl. LXXXIII.

1916. nemoralis, Thw.

IV.51

719. Ostodes, Bl.

1917. zeylanica, Muell. Arg. Wal-keluna,

Olupetta, S.

IV.52 var. $\beta$ minor, Thw.

720. Blachia, Baill.

1918. umbellata, Baill. Kosatta, s.

IV.53

721. Dimorphocalyx, Thw.

1919. glabellus, Thw. Weli-wenna, S. Tentukki, T.

IV.54, Pl. LXXXIV.

722. Agrostistachys, Dalz.

1920. indica, Dalz.

1921. HOOKERI, Benth. Maha-beru, Diya-

beru, S.

IV.55

1922. longifolia, Benth. Beru, S.

IV.55

IV.56

723. Chrozophora, Neck.

1923. plicata, A. Juss.

IV.56

724. Acalypha, L.

1924. paniculata, Miq.

IV. 57

1925. fruticosa, Forsk.

IV.58

1926. indica, L. Kuppa-meniya, S. Kuppameni, Punairananki, T.

IV.58

1927. brachystachya, Hornen.

IV.59

1928. lanceolata, Willd..

IV.59

1929. ciliata, Forsk.

IV.59

725. Adenochlæna, Boiv.

1930. zeXlanica, Thw. ${ }^{1} \quad$ IV.60, Pl. LXXXV.

1 Probably worthy of rank as an endemic genus (Centrostylis, Baill), according to Hookor. 
726. Trewia, L.

1931. nudiflora, $\mathrm{L}$.

IV.61

727. Tragia, L.

1932. involucrata, L. Wel-kahambiliya, S.

IV.61 var. $\beta$ cordata, Muell. Arg. var. $\gamma$ cannabina, Hk. $f$.

728. Podadenia, Thw.

1933. SAPIDA, Thw.

IV.62

729. Claoxylon, A. Juss.

1934. Mercurialis, Thw.

1935. oligandrom, Muell. Arg.

IV.63

IV.64

730. Mallotus, Lour.

1936. albus, Muell. Arg. Bu-kenda, S. IV.64

1937. erIocarPus, Muell. Arg. IV.65

1938. WALKER丑, Hk. f.

IV.66

1939. rhamnifolius, Muell. Arg. Marai-tinni, T.

var. $\beta$ ovalifolius, Hk. $\mathrm{f}$.

1940. Fuscescens, Muell. Arg.

1941. distans, Muell. Arg.

1942. repandus, Muell. Arg.

1943. philippinensis, Muell. Arg. Hamparila,

S. Kapila, T.

IV.66

IV. 67

IV.67

IV.67

731. Cleidion, Bl.

1944. javanicum, Bl. Okuru, S. IV.69

1945. nitidum, Thw.

IV.69

732. Macaranga, Thou.

1946. indica, Wight. Vattakanni, T.

1947. tomentosa, Wight. Kenda, Pat-kenda, $\mathrm{S}$.

IV.68

948. DIGYna, Muell. Arg. Ota, Gal-ota, S.

IV.70

IV.70

IV.71

733. Homonoia, Lour.

1949. riparia, Lour.

IV.72

734. Dalechampia, L.

1950. indica, Wight.

IV.72

735. Gelonium, Roxb.

1951. lanceolatum, Willd. Kakkaipalai, Varittulai, Potpattai, T.

IV.73

736. Chætocarpus, Thw.

1952. castanocarpus, Thw. Hedoka, Hedawaka, S.

IV.74

1953. PUBESCENS, Hk. f.

IV.74

1954. CORIACEus, Thw. Hedoka, Hedawaka, s. IV.75 
737. Sapium, P. Br.

1955. indicum, Willd. Kiri-makulu, s. IV.75

1956. insigne, Trim. Tel-kaduru, S. Tilai, T. IV.76

738. Excœcaria, L.

1957. Agallocha, L. Tela-kiriya, S.

IV.77

1958. crenulata, Wight.

IV.77

739. Sebastiania, Spreng.

1959. Chamælea, Muell. Arg. Rat-pitawakka,

S.

IV.78

\section{Urticaceæ.}

740. Holoptelea, Planch.

1960. integrifolia, Planch. Goda-kirilla, S. Ayil, Velayil, Kanchia, T. Indian elm.

IV.80

741. Celtis, L.

1961. cinnamomea, Lindl. Gurenda, S. IV.8I,

1962. Wightii, Planch. Meditella, S.

IV.81

742. Trema, Lour.

1963. orientalis, Bl. Gedumba, S. Charcoal tree.

IV.82

743. Gironniera, Gaudich.

1964. subæqualis, Planch. var. zeylanica, Thw. Akmediya, S.

1965. reticulata, Thw. Wal-munamal, S.

IV.83

IV.83

744. Ficus, L.

1966. parasitica, Kcen. Gas-netul, Wel-ehetu, S.

IV.85

*1967. benghalensis, L. Maha-nuga, S. $A l$,

T. Banyan.

IV.86

1968. mysorensis, Heyne. Bu-nuga, S.

IV.86

1969. tomentosa, Roxb. Wel-aralu, S.

1970. altissima, Bl. var. Fergusoni, King.

Nuga, Kosgona, S.

1971. Trimeni, King.

1972. CaUdiculata, Trim.

1973. retusa, L. Panu-nuga, S. Itti, T.

1974. nervosa, Heyne. Kalumaduwa, S.

1975. Arnottiana, Miq. Kaudu-bo, S.

1976. MOONIANA, King.

1977. Tsjakela, Burm. Kiri-pella, S.

[V.87

1978. Tsiela, Roxb. Ela-nuga, Ehetu, S. Kalatti, T.

IV.87

IV.88

IV. 88

IV.89

IV.89

IV.90

IV.91

IV.91

IV.92

IV.92

1979. infectoria, Roxb. var. \& Lambertiana, King. Kalaha, S. 
1980. callosa, Willd. Wat-gona, s.

IV .93

1981. heterophylla, L. f. Wal-ehetu, s.

1982. asperrima, Roxb. Sewana-mediya, S. Furniture leaf.

1983. hispida, L. f. Kota-dimbula, S.

1984. ThWAITESII, Miq.

1985. lævis, Bl. var. dasyphylla, King.

IV.93

1986. glomerata, Roxb. Attikka, S. Atti, T. IV.96

745. Antiaris, Leschen.

1987. toxicaria, Leschen. Riti, S. Netavil, T. Upas tree.

IV.97

746. Cudrania, Trec.

1988. javanensis, Trec. IV.98

747. Artocarpus, Forst.

1989. NoBILIs, Thw. Del, Bedi-del, S. IV.98

1990. Lakoocha, Roxb. Kana-gona, S. IV.99 var. $\beta$ Gomeziana, Wall. (sp.)

748. Taxotrophis, Bl.

1991. zeylanica, Thw.

IV.100

749. Phyllochlamys, Bureau.

1992. spinosa, Bureau. Gon-gotu, S. IV.101

750. Streblus, Lour.

1993. asper, Lour. Geta-netul, S. Patpirai, Pirasu, T.

IV.101

751. Dorstenia, L.

1994. indica, Wall. IV.102

752. Allæanthus, Thw.

1995. zeYlanicus, Thw. Alandu, S. IV.103

753. Plecospermum, Trecul.

1996. spinosum, Trec. Katu-timbol, s. $\quad$ IV.103

754. Fleurya, Gaudich.

1997. interrupta, Gaudich. Wal-kahambiliya, S.

IV.104

755. Laportea, Gaudich.

1998. terminalis, Wight.

1999. crenulata, Gaudich. Maussa, S. Devilnettle, Fever-nettle.

IV.105

IV.105

756. Girardinia, Gaudich.

2000. heterophylla, Dene. Gas-kahambiliya,

S. Nilgiri-nettle.

IV.106 var. $\beta$ palmata, Hk. f. 
757. Pilea, Lindl.

2001. Wightii, Wedd.

2002. stipulosa, Miq.

IV.107

2003. trinervia, Wight.

IV.107

IV.108

758. Lecanthus, Wedd.

2004. Wightii, Wedd.

IV.108

759. Pellionia, Gaudich.

2005. Heyneana, Wedd.

IV.109

760. Elatostema, Forst.

2006. WALKER $A$, Hk. f.

2007. acuminatum, Brongn.

IV.110

2008. lineolatum, Wight.

var. $\beta$ lineare, Wedd.

var. $\gamma$ bidentatum, Hk. f.

var. $\delta$ falcigerum, Wedd.

var. $\varepsilon$ petiolare, Thw.

2009. surculosum, Wight. var. $\beta$ rigidiusculum, Thw.

IV.111

761. Procris, Juss.

2010. lævigata, Bl.

IV.112

762. Bœhmeria, Jacq.

IV.110

IV.110

2011. malabarica, Wodd. Maha-diya-dul, S. IV.113

2012. platyphylla, Don.

IV.114

763. Chamabainia, Wight.

2013. cuspidata, Wight. ' IV.114

764. Pouzolzia, Gaudich.

2014. indica, Gaudich. var. $\beta$ alienata, Wedd.

2015. auriculata, Wight. var. $\beta$ bicuspidata, Hk. $f$.

IV.115

IV.116

2016. WALKERIANA, Wight.

IV.116

2017. Bennettiana, Wight.

IV.117

var. $\beta$ Gardneri, Wedd.

2018. parvifolia, Wight.

IV.117

765. Villebrunea, Gaudich.

2019. integrifolia, Gaudich. var. sylvatica, Hk. f. IV.118

766. Debregeasia, Gaudich.

2020. velutina, Gaudich, Gas-dul, S. Wild

rhea.

2021. ZEYLANICA, Hk. f.

IV.119

IV.119

122. Ceratophyllaceæ.

767. Ceratophyllum, L.

2022. verticillatum, Roxb. 


\section{( 86$)$ \\ GYMNOSPERM F.}

123. Cycadaceæ.

768. Cycas, L.

2023. circinalis, L. Madu, S.

IV.121

*2024. Rumphii, Miq. Maha-madu, S.

IV.122

\section{MONOCOT YLEDONS.}

\section{Hydrocharitaceæ.}

769. Hydrilla, Rich.

2025. ovalifolia, Rich.

IV 123

770. Lagarosiphon, Harv.

2026. Roxburghii, Benth.

IV.124

771. Blyxa, Thou.

2027. zeYLanica, Hk. f. Diyahawari, S.

IV.125

772. Ottelia, Pers.

2028. alismoides, Pers.

IV.125

773. Enhalus, Rich.

2029. Kœnigii, Rich.

IV.126

774. Thalassia, Soland.

2030. Hemprichii, Aschers. Chatelai, T.

IV.127

775. Halophila, Thou.

2031. ovata, Gaudich.

2032. sp. nov. (Chilaw). ${ }^{1}$

IV.128

2033. Beccarii, Aschers.

IV.129

IV.129

125. Burmanniaceæ.

776. Burmannia, L.

2034. disticha, L. Mediya-jawala, S. IV.130

2035. cœlestis, Don.

IV.131 var. $\beta$ pusilla, Trim.

2036. CHAMPIONII, Thw.

1V.131, Pl. LXXXVII.

777. Thismia, Griff.

2037. GARDNERIANA, Hk. f.

IV.132

126. Orchidaceæ.

778. Oberonia, Lindl.

2038. TRUNCATa, Lindl.

IV.136

2039. recurva, Lindl.

IV.137

2040. THWAITESII, Hk. f:

IV.137

2041. LONGIBRACTEATA, Lindl.

IV.138

1 Cf. Hooker in Trimen's Flora, IV., p. 129. 
2042. ZEYLANICA, Hk. f.

2043. TENUIS, Lindl.

2044. FORCIPATA, Lindl.

IV.138

IV.138

2045. Wightiana, Lindl.

IV.139

2046. SCYLI,E, Lindl.

IV.139

IV.139

779. Microstylis, Nutt.

2047. PURPUREA, Lindl. (?? also Java.)

IV.140

2048. DISCOLOR, Lindl.

2049. congesta, Rchb. f.

IV.141

2050. Rheedii, Wight.

2051. versicolor, Wight.

IV.14]

2052. LANCTFOLIA, ThW.

IV.14]

IV.142

IV.142

780. Liparis, Rich.

2053. THWAITESII, Hk. f.

2054. Wightiana, Thw:

2055. TRIMENII, Ridley.

2056. BARBATA, Lindl.

2057. nervosa, Lindl.

2058. Walkeriæ, Graham.

2059. atropurpurea, Lindl.

2060. BRACHYGLOTTIS, Rehb. f.

2061. OBSCURA, Hk. f.

2062. longipes, Lindl.

2063. viridiflora, Lindl.

2064. disticha, Lindl.

IV.143

IV.144

IV.144

IV.145

IV.145

IV.146

IV. 146

IV.147

IV.147

IV.147

$1 V .148$

IV.148

781. Dendrohium, Swartz.

2065. Macræi, Lindl. Juta-makuta, S.

IV. 150

2066. PANDURATUM, Lindl.

$1 V .150$

2067. DIODON, Rchb. f.

2068. crumenatum, Sw. Sudu-pareyi-mal, S.

White dove orchid.

IV.151

2069. nutans, Lindl.

2070. macrostachyum, Lindl.

IV.15I

IV.152

2071. hæmoglossum, 'l'hw.

$1 V .152$

2072. MaCaRTHIж, Thw. Wesak-mal, S.

IV.152

IV.15:

2073. heterocarpum, Wall. Primrose orchid. IV.154

782. Pulbophyllum, Thou.

2074. CRASSIFOLIUM, 'Thw.

IV.155

2075. PETIOLARE, Thw.

IV.155

2076. PURPUREUM, Thw.

2077. eLegans, Gardn.

IV.155

2078. SP. Nov. (Ritigala). ${ }^{1}$

IV.156, PI. LXXXVIII.

${ }^{1}$ Willis, Flora of Ritigala, Ann. Perad., III., p. 287.

$6(11) 10$ 
783. Cirrhopetalum, Lindl.

2079. GRANDIFLORUM, Wight.

IV.157

2080. wightiI, Thw.

IV.157

2081. TRIMENI, Hk. f.

IV.158

2082. MACR ÆI, Lindl.

1V.158

2083. ThWAITESII, Relib. f.

IV.159

784. Cologyne, Lindl.

2084. BREVISCAPA, Lindl.

IV. 160 2085. odoratissima, Lindl. 2086. ZEYLANICA, Hk. f.

[V.160, Pl. I.XXX]X.

IV.161

785. Adrorhizon, Hk. f.

2087. PURPURASCENS, Hk. f.

IV.161

786. Pholidota, Lindl.

2088. imbricata, Lindl.

IV.162

787. Chrysoglossum, $\mathrm{Bl}$.

2089. MACULATUM, Hk. f.

IV.163

788. Acanthephippium, Bl.

2090. BICOLOR, Lindl.

IV.164

789. Eria, Lindl.

2091. BRACCATA, Lindl.

IV.165

2092. muscicola, Lindl.

IV.165 var. $\beta$ oblonga, Trim.

2093. BICOLOR, Lindl. Lily of the valley orchid. IV.166 2094. TRICOLOR, Thw.

2095. LINDLEYI, Thw.

IV.166

IV.167

2096. ThWaItesir, Trim.

IV.167

790. Alvisia, Lindl.

2097. TENUIS, Lindl.

IV.168

791. Tainia, Bl. 2098. bicornis, Trim.

IV.169

792. Arundina, Bl.

2099. MTNOR, Lindl.

IV.170

793. Agrostophyllum, Bl.

2100. ZEYLANICUM, Hk. f.

IV.171

794. 1psea, Lindl.

2101. spectosa, Lindl. Daffodil orchid.

IV.171

795. Phains, Lour.

2102. WALIICHII, Lindl.

2103. LURIDUs, Thw.

IV.172

IV.173

796. Calanthe, $\mathrm{Br}$.

2104. PURPUREA, Lindl.

2105. veratrifolia, $\mathrm{Br}$.

IV.174

IV.174 var. $\beta$ discolor, Lindl. 
797. Euloplia, Br.

2106. virens, Br.

2107. graminea, Lindl.

[V.175

2108. macrostachya, Lindl.

IV.176

2109. nuda, Lindl.

IV.176

2110. sanguinea, Hk. f.

IV.177

IV.177

798. Geodorum, Jacks.

2111. dilatatum, Br.

IV.178

799. Cymbidium, Swartz.

2112. bicolor, Lindl.

IV.179

2113. ensifolium, Sw. var. hæmatodes, 'T'rim. IV.180,

Pl. XC.

800. Josephia, Wight.

2114. lanceolata, Wight.

2115. latifolia, Wight.

IV.182

IV.182

801. Polystachya, Hk.

2116. ZEYLANICA, Lindl.

IV.183

802. Sarcochilus, $\mathrm{Br}$.

2117. Wightii, Hk. f.

2118. VIRIDIFLORUS, Hk. f.

2119. PULCHELluS, T'Tim.

2120. PUGTONIFOLIUS, Hk. $f$.

2121. Complanatus, Hk. f.

IV.184

IV.184

IV.185

IV.185

IV.186

803. Rhynchostylis, $\mathrm{Bl}$.

2122. retusa, Bl. Fox-tail orchid, Batticaloa orchid.

IV.187

804. Doritis, Lindl.

2123. Wightii, Benth.

IV.188

805. Atrides, Lour.

2124. cylindricum, Lindl.

2125. lineare, Hk. f.

IV.189

IV.189

806. Luisia, Gaudich.

2126. teretifolia, Gaudich.

2127. tenuifolia, $\mathrm{Bl}$.

IV.190

IV.191

807. Vanda, Br.

2128. parviflora, Lindl.

2129. Roxburghii, Br.

IV.192

2130. THWAITESII, Hk. f.

IV.192

2131. spathulata, Spreng.

IV.193

[V.193

sos. Diplocentrum, Lindl.

213\%. recurvum, lindl. 
809. Saccolabium, Bl.

2133. NIVEUM, Lindl.

2134. filiforme, Lindl.

2135. GRACILE, Lindl.

2136. BREVIFOLIUM, LindI.

2137. ROSEUM, Lindl.

2138. ochraceum, Lindl.

2139. ACAULE, Hk. f.

2l40. longifolium, Hk. f.

2141. Wightianum, Hk.f.

IV.195

IV. 196

IV.196

IV.196

IV.197

IV.197

IV.198

IV.198

IV.199

810. Sarcanthus, Lindl.

2142. peninsularis, Dalz.

IV. 200

811. Cleisostoma, B1.

2143. MACULOSUM, Lindl.

IV. 200

2144. tenerum, Hk. f.

IV.201

2145. DECIPIENS, Lindl.

IV.201

812. Mystacidium, Lindl.

2146. ZEYLANICUM, Trim.

IV.202

813. Cottonia, Wiglit.

2147. macrostachya, Wight.

IV.20:3

81.t. Tæniophyllım, Bl.

2l 48. ALWISII, Lindl.

IV.203

815. Diploprora, Hk. f.

2149. Championii, Hk. f.

$1 V .204$

s16. Podochilus, Bl.

2150. FALCATUS, Lindl.

IV.205

2151. nalabariciss, Wight. IV.206

2152. saxatilis, Lindl.

IV.206

817. Phreatia, Lindl.

2153. elegans, Lindl.

IV.207

818. Octarrhena, 'Thw.

2154. PaRvUla, Thw.

IV.208

819. C'ryptostylis, $\mathrm{Br}$.

2155. Arachnites, Bl.

IV.209

820. Hetæria, B1.

2156. GARDNERI, Benth.

IV.209

2157. elongata, Lindl.

IV.210

821 . Cheirostylis, $\mathrm{Bl}$.

2158. PARVIFOLIA, Lindl.

2159. flabellata, Wight.

IV.211

IV.211

82.2. Pliysurus, Rich.

2160. Blumei, Lindl.

IV.2I 2 


\section{$(91)$}

823. Anrectochilus, BI.

2161. REGalis, Bl. Wana-raja, S. TV.213

82t. Goodyera, $\mathrm{Br}$.

2162. procera, Hk. f.

IV.214 2163. fumata, Thw.

IV.214

82.5. Zeuxine, Lindl.

2161. sulcata, Lindl.

IV.215

2165. longilabris, Benth.

IV. 216

2166. REGIA, Benth. Iru-raja, S.

IV. 216

2167. flava, Benth.

IV.217

8.6. Spiranthes, Rich.

2168. australis, Lindl.

IV.217

827. Corymbis, Thou.

2169. veratrifolia, B1.

IV.218

828. Tropidia, Lindl.

2170. THWAITESII, Hk. f.

IV.219 var. $\beta$ major, Hk. $f$.

2171. BaMmusifolia, Trim.

IV. 220

529. Vainilla, Sw.

2172. Walkerix, Wight.

2173. MooNII, Thw.

$1 V .220$

IV.221, PI. XCI.

830. Gastrodia, Br.

2174. javanica, Lindl.

IV.221

831. Epipogum, Gmel.

2175. nutans, Lindl.

[V.22.2

832. Galeola, Lour.

2176. javanica, Benth.

IV.223

833. Aphyllorchis, $\mathrm{Bl}$.

2177. montana, Relib. f.

IV.224

834. Pogonia, Griff.

2178. juliana, Wall. (?)

IV.225

835. Habenaria, Willd.

2179. barbata, Wight.

2180. acuminata, Trim.

IV. 226

2181. macrostachya, Lindl.

IV. 227

2182. DOLICHOSTACHYA, Thw.

IV. 227

2183. DICHOPETALA, Thw.

2184. plantaginea, Lindl. Pigeon orchid.

IV. 228

IV. 228

2185. crinifera, Lindl.

IV. 229

IV. 229

2186. PTEROCARPA, Thw.

IV. 230

2187. RHY NCOCARPA, Trim.

IV.230

2188. viridiflora, $\mathrm{Br}$.

IV.231

2189. BREVILOB A, 'I'rim.

IV.232 
2190. Wightii, Trim.

IV. 232

2191. aristata, Trim.

IV. 233

2192. TRIMENI, Hk. f.

IV. $23: 3$

2193. torta, Hk. f.

IV.234

2194. GARDNERI, Hk. f. var. $\beta$ latifolia, Hk. f.

IV.234

2195. cubitalis, $\mathrm{Br}$.

IV.235

836. Disperis, Sw.

2196. zeylanica, T'rim.

IV.236

837. Satyrium, Sw.

2197. nepalense, Don. Hyacinth orchid.

IV.237

838. Apostasia, Bl.

2198. Wallichii, Br.

IV.238

127. Scitamineæ.

839. Globba, L.

2199. bulbifera, Roxb.

IV.240

840. Curcuma, L.

2200. aromatica, Salisb. Dada-kaha, $\mathrm{Wal}$ kaka,

*2201. Zedoaria, Roscoe. Maran-kaha, S.

IV.241

IV.241 2202. OLIGANTHA, Trim.

2203. ALBIFLORA, Thw.

IV.242, P], XCII.

IV.242

841. Kæmpferia, L.

*2204. pandurata, Roxb. Amba-kaha, S.

IV.243

*2205. rotunda, L. Yawakenda, Lankenda, S.

IV.244

842. Hedychium, Kœn.

2206. coronarium, Kœen. Ela-mal, S.

IV. 245

2207. flavescens, Carey.

2208. coccineum, Ham.

IV.245

IV.246

843. Costus, L.

2209. speciosus, Sm. Tebu, S.

IV.246

844. Alpinia, L.

2210. Allughas, Rosc. Alu, Alu-gus, Alan, Keleniya, S.

2211. nutans, Rosc. var. sericea, Moon.

Rankiriya, S.

IV.247

2212. RUFESCENS (Thw.), K. Schun.

IV.248

IV.256

845. Amomum, L.

2213. FLORIBUNDUM, Trim.

IV.250

2214. INVOLUCRATUM, Trim.

IV.250

2215. NEMORALE, Trim.

IV.251

2216. ACUMTNATUM, Thw.

IV.251

2217. FuLviCEPS, 'Thw.

IV. 252 


\section{$\left(\begin{array}{l}93\end{array}\right)$}

2218. Masticatorium, Thw.

IV.252

2219. GRAMINIFOLIUM, 'Thw.

IV.253

2220. ciliatum, Baker.

IV. 253

2221. hypoleucum, Thw.

IV.254

2222. PTEROCARPUM, Thw.

2223. echinatun, Willd. (" Malaya.)

IV.254

2224. Benthamianum, Trim.

IV.255

IV.255

846. Zingiber, Adans.

2225. Wightianum, Thw.

IV.257

2226. CYLINDRICUM, Moon.

IV. 257

*2227. Cassumunar, Roxb.

IV.258

*2228. Zerumbet, Smith. Walinguru, S.

IV.259

847. Cyphostigma, Benth.

2229. Pulchellum, Benth. ${ }^{1}$

IV.260

848. Elettaria, Maton.

2230. Cardamomum, Naton. var. major,

Smith. Ensal, S. Cardamom.

IV.261

849. Clinogyne, Salisb.

2231. virgata, Benth. Creta-oluwa, S.

IV.262

850. Phrynium, Willd.

2232. zeylanicui, Benth. Hulan-kiriya, S. IV.263

2233. capitatum, Willd. Et-bemi-kiriya, S. IV.263

851. Canna, L.

2234. indica, L. But-sarana, S. Indian shot. IV.264

852. Musa, L.

2235. paradisiaca, L. Kehel, Gal-kehel, S.

Wild plantain.

IV.265

128. Hæmodoraceæ.

853. Ophiopogon, Ker.

2236. intermedius, Don.

IV.267

854. Sansevieria, Thunb.

2237. zeylanica, Willd. Niyanda, S. Maral, $\mathrm{T}$.

IV. 267

129. Amaryllidaceæ.

855. Curculigo, Gærtn.

2238. Finlaysoniana, Wali. Ma-bin-tal, S. IV.269 var. $\beta$ linearifolia, 'Thw.

2239. orchioides, Ciedn. IIn-bin-tal, S.

Nilappanai, T.

IV.269

I new sp., pedicellatum. K. Schum., split of from this in Das Pflanzenr $\theta i c h$. 


\section{( $94 \quad)$}

856. Crinum, L.

2240. asiaticum, L. Tolabo, S. Vichamunkil, $\mathrm{T}$.

IV.270

2241. defixum, Ker. Hin-tolabo, S.

2242. latifolium, L. Tolabo, S. Vichamunkil, T.

IV.271

IV.271

var. $\beta$ zeylanicum, Hk. f.

857. Pancratium, L.

2243. zeylanicum, L. Wal-lunu, S.

IV.272

130. Taccaceæ.

858. Tacca, Forst.

2244. pinnatifida, J. \& G. Forst. Garandi-

kidaran, S.

IV.274

131. Dioscoreaceæ.

859. Dioscorea, L.

2245. tomentosa, Heyne. Uyala, S. IV.275 2246. pentaphylla, L. Katuwala, S. Allai, T. IV.276 2247. oppositifolia, L. Hiritala, S. IV.276 2248. INTERMEDIA, Thw. IV.277 2249. spicata, Roth. Gon-ala, S. IV.277, Pl. XCIII: 2250. sativa, L. Panu-kondol, S. IV.278

860. Trichopus, Gærtn.

2251. zeylanicus, Gærtn. Bim-pol, S.

IV. 280

\section{Roxburghiaceæ.}

861. Stemona, Lour.

2252. minor, Hk. f.

IV.281

133. Liliaceæ.

862. Smilax, L.

2253. aspera, L. IV.283

2254. zeylanica, L. Kabarasa, Hin-kabarasa, S. IV.283

2255. prolifera, Roxb. Maha-kabarasa, S. IV.283

863. Asparagus, L.

2256. racemosus, Willd. Hatawariya, $\mathrm{S}$.

Chattavari, T.

IV. 285

2257. ZEYLANICUS, Hk. f.

2258. falcatus, L. Hatawariya, S.

IV. 285

2259. gonoclados, Baker.

IV.285

IV.286

864. Dracæna, L.

2260. Thwaitesii, Regel.

IV.287

865. Dianella, Lam.

2261. ensifolia, Redouté. Monara-petan, S. IV.28s 
866. Disporum, Salisb.

2262. Leschenaultianum, D. Don.

IV.289

867. Chlorophytum, Ker.

2263. Heyneanum, Wall.

IV.289

2264. laxum, $\mathrm{Br}$.

IV. 290

868. Allium, L.

2265. Hookeri, Thw.

IV.291

869. Dipcadi, Medic.

2266. montanum, Baker.

IV.291

870. Urginea, Steinheil.

2267. RUPICOLA, Trim.

IV.29:2

871. Scilla, L.

2268. indica, Baker.

IV.293

872. Iphigenia, Kunth.

2269. indica, A. Gray.

IV.293

873. Gloriosa, L.

2270. superba, L. Niyangala, S. Karttikai-

kilanku, Ventonti, T.

IV.294

134. Pontederiaceæ.

874. Monochoria, Presl.

2271. Lastæfolia, Presl. Diya-habarala, S.

2272. vaginalis, Presl.

IV.295

IV.295

var. $\beta$ plantaginea, Solms.

\section{Xyridaceæ.}

875. Xyris, L.

2273. indica, L. Ran-motu, S.

IV.297

2274. anceps, Lam.

IV.297

2275. schœnoides, Mart.

IV. 297

2276. pauciflora, Willd.

IV.298

136. Commelinaceæ.

876. Pollia, Thunb.

2277. sorzogonensis, Endl.

IV.299

877. Commelina, L.

2278. nudiflora, L. Girapala, S. IV.300

2279. benghalensis, L. Diya-meneriya, S. IV.301

2280. clavata, Clarke. Girapala, S.

IV.301

2281. persicariæfolia, Wight.

IV.302

2282. THWAITESII, Hk. f.

IV.302

2283. attenuata, Vahl.

IV.303

2284. obliqua, Ham.

IV.303

$6(11) 10$ 


\section{( 96$)$}

2285. Kurzii, Clarke.

IV.304

2286. ensifolia, Br.

IV.304

2287. appendiculata, Clarke.

IV.304

878. Aneilema, Br.

2288. glaucum, Thw.

IV.305

2289. esculentum, Wall.

2290. zeylanicum, Clarke.

IV.306

2291. dimorphum, Dalz.

IV.306

2292. spiratum, Br.

IV.307

2293. nudiflorum, Br. var. $\beta$ terminale, Clarke.

IV.307

IV.308

2294. giganteum, $\mathrm{Br}$.

IV.308

2295. vaginatum, $\mathrm{Br}$.

IV.309

2296. montanum, Wight.

IV.309

2297. protensum, Wall.

IV.310

879. Cyanotis, Don.

2298. eristata, Schultes f. Bol-hinda, S.

IV.311

2299. овTUSA, Trim.

IV.312, Pl. XCIV.

2300. tuberosa, Schultes f. var. adscendens,

Clarke

2301. ZEYLANiCA, Hassk.

2302. villosa, Schultes f.

2303. fasciculata, Schultes f.

2304. pilosa, Schultes f.

2305. axillaris, Schultes f.

IV.312

IV.313

IV.313

IV.314

IV.314

IV.315

880. Floscopa, Lour.

2306. scandens, Lour.

IV.316

137. Flagellariaceæ.

881. Flagellaria, L.

2307. indica, L. Goyi-wel, S.

IV.317

882. Susum, Bl.

2308. anthelminticum, Bl. Induru, S.

IV.317

138. Juncaceæ.

883. Juncus, L.

2309. effusus, L.

2310. prismatocarpus, Br.

IV.318

IV.319

139. Palmaceæ.

884. Areca, L.

2311. Catechu, L. Puwak, S. Kamukai, T.

Arecanut palm.

IV.321

2312. concinna, Thw. Len-teri, S.

IV.322 
885. Loxococcus, Wendl. \& Drude.

2313. RuPICOLA, Wendl. \& Drude. Dotalu, S. IV.322

886. Oncosperma, Bl.

2314. Fasciculatum, Thw. Katu-kitul, S. IV.323

887. Caryota, L.

2315. urens, L. Kitul, S. Tippilipana, T.

Toddy palm.

IV.324

888. Nipa, Wurmb.

2316. fruticans, Wurmb. Gin-pol, S.

IV.325

889. Phœnix, L.

2317. ZeYlanica, Trim. Indi, S. IV.326, Pl. XCV. 2318. pusilla, Gærtn. Inchu, T.

IV. 327

890. Corypha, L.

2319. umbraculifera, L. Tala, S. Talipot. IV.328

891. Calamus, L.

2320. Thwaitesii, Becc.

2321. pseudo-tenuis, Becc.

IV.330

IV.330

2322. Rotang, L. Wewel, S. Priampu, T. IV.331

2323. RIVALIS, Thw. Ela-wel, S.

IV.332

2324. Delicatulus, Thw. Nara-wel, S.

2325. RAdiatus, Thw. Kukula-wel, S.

IV.332

2326. PaChystemonUs, Thw.

2327. Digitatus, Becc. Kukula-vel, S.

IV.333

IV.333

2328. ZeYlanicus, Becc. Ma-wewel, Wanderu-

wel, S.

2329. ovordeus, Thw. Tambutu-wel, S.

IV.334

IV.335

IV. 335

892 Borassus, L.

*2330. flabellifer, L. Tal, S. Panai, T.

Palmyra palm.

IV .336

893. Cocos, L.

*2331. nucifera, L. Pol, S. Tennai, T.

Coconut palm.

IV.337

140. Pandanaceæ.

894. Pandanus, L. f.

2332. odoratissimus, L. f. Mudu-keyiya, S.

Talai, T. Screw-pine.

2333. ZEYlanicus, Solms. O-keyiya, S.

IV.339

IV.339

2334. fœtidus, Roxb. var. racemosus, Kurz.

Dunu-keyiya, S.

IV. 340

895. Freycinetia, Gaudich.

2335. PYCNOPHYLLA, Solms.

IV. 341

2336. WALkEIr, Solms.

IV.342 


\section{Typhaceæ.}

896. Typha, L.

2337. javanica, Schnitzl. Hambu-pan, S. IV.343

142. Araceæ.

897. Pistia, L.

2338. Stratiotes, L. Diya-parandella, S.

Water lettuce.

IV.345

898. Cryptocoryne, Fisch.

2339. spiralis, Fisch.

2340. THWAITESII, Schott.

IV.346

2341. NEVILLII, Trim.

IV.346

2342. WALKERI, Schott.

IV.346

2343. BECKETTII, Thw.

IV.347

IV.347

899. Lagenandra, Dalz.

2344. THWAItesir, Engl.

2345. LANCIFolia, Thw. Ati-udayan, S.

IV.348

2346. toxicaria, Dalz. Vetala, S.

IV.348

2347. KENIGII, Thw.

IV.349

2348. INSIGNIS, Trim.

IV.349

IV.350

900. Arisæma, Mart.

2349. neglectum, Schott. Wal-kidaran, S.

IV.351

2350. FILICA UDATUM, N. E. Br.

IV.351

2351. Leschenaultii, Bl. Wal-kidaran, S.

IV.352

901. 'Typhonium, Schott.

2352. trilobatum, Schott. Panu-ala, S.

2353. Roxburghii, Schott. Polon-ala, S.

IV.353

2354. cuspidatum, Dene.

IV.353

IV.354

902. Theriophonum, Bl.

2355. CRENATUM, Bl.

IV.355

903. Amorphophallus, Bl.

2356. campanulatus, Bl. Kidaran, S.

2357. DUBIUS, Bl.

IV.355

IV.356

904. Synantherias, Schott.

2358. sylvatica, Schott.

IV.357

905. Remusatia, Schott.

2359. vivipara, Schott.

IV.358

906. Colocasia, L.

2360. Antiquorum, Schott. Gahala, S. Taro. IV.359

907. Alocasia, Schott.

*2361. cucullata, Schott. Panu-habarala, S. IV.360

*2362. macrorrhiza, Schott. Habarala, S. 
908. Raphidophora, Schott.

2363. pertusa, Schott.

IV.361

2364. decursiva, Schott. Dada-kehel, S. IV.362

909. Lasia, Lour.

2365. aculeata, Lour. Kohila, S.

IV.363

910. Pothos, L.

2366. oEYlanicus, Engl.

2367. scandens, L. Pota-wel, S. IV.364

2368. HOOKERT, Schott.

IV.364

2369. REMOTIFLURUS, Hk.

IV.364

911. Acorus, L. var. $\beta$ macrophylla, Hk. $\mathrm{f}$.

*2370. Calamus, L. Wada-kaha, S. Sweet flag. IV.365

\section{Lemnaceæ.}

912. Lemna, L.

2371. paucicostata, Hegeln. Diya-panshi, S. IV.366 2372. polyrrliza, L.

IV.367

913. Wolffia, Heckel.

2373. Michelii, Schleid. (arrhiza, Wimm.)

IV.367

\section{Triuridaceæ.}

914. Sciaphila, B1.

2374. ERUBescens, Miers.

2375. SECUNDIFLORA, Thw.

IV.368

2376. janthina, Thw.

IV.368

IV.369

145. Alismaceæ.

915. Alisma, L.

2377. oligococcum, F. Muell.

IV.370

916. Limnophytum, Miq.

2378. obtusifolium, Miq.

IV.370

\section{Naiadaceæ.}

917. Aponogeton, L. f.

2379. natans, Engl. \& Krause (monostachyon,

L. f.). Koddi, T.

IV.372 2380. CRISPUM, Thunb. Kekatiya, S.

IV.372

918. Potamogeton, L.

2381. indicus, Roxb.

2382. pectinatus, $\mathrm{L}$.

IV.373

IV.374

919. Ruppia, L.

2383. maritima, L. var. rostellata, Græbn. IV.374 
920. Najas, L.

2384. marina, L. (major, All.)

IV.375

2385. graminea, Del.

IV.375

2386. minor, All.

IV.376

921. Cymodocea, Kon.

2387. serrulata, Aschers. \& Magn.

IV.376

2388. isøtifolia, Aschers.

IV.377

922. Diplanthera, Thou.

2389. uninervis, Aschers. (Cymodocea australis, Trim.)

147. Eriocaulonaceæ.

923. Eriocaulon, $I_{4}{ }^{1}$

2390. setaceum, L. Penda, S.

2391. Capillus-naiadis, Hk. f.

V.2

2392. CAULESCENS, Hk. f. \& Th.

V.3

2393. 7.EYLANICUM, Kœrn.

2394. LONGICUSPIS, Hk. f.

V.3

2395. atratum, Korn.

V.4

2396. sexangulare, L. Kokmota, S.

V.4

2397. Thwaitesii, Koern.

V.5

2398. Brownianum, Mart.

V.6

2399. luzulæfolium, Mart.

V.6

2400. truncatum, Ham.

V.7

V.7

2401. TRIMENI, Hk. f.

V.8

2402. Wightianum, Mart.

V.8

2403. WALKERI, Hk. f.

2404. quinquangulare, L. Hin-kokmota, S. V.9

2405. collinum, Hk. $\mathrm{f}$.

V.10

2406. Sieboldianum, Sieb. \& Zuce.

V.10

2407. hluviatile, Trim.

V.11

\section{Cyperaceæ.}

924. Cyperus, L. ${ }^{2}$

2408. Cephalotes, Valll.

2409. Iria, L. Wel-hiri, S.

2410. pygmæus, Rottb.

2411. stramineus, Nees.

2412. pumilus, L. Go-kivi, S.

1 Cf. Ruhland in Das Pflanzenreich, who makes new spp. and re-arranges these. I have preforred Hooker's arrangement however.

${ }^{2}$ I follow 'Trimen here for convenience salie, though Pycreus at any rate should, I think, be separated from Cyperus. 
2413. hyalinus, Vahl.

2414. sanguinolentus, Vahl.

2415. polystachyus, Rottb.

V.20

2416. puncticulatus, Vahl.

V.21

2417. globosus, Allioni.

V.21

2418. bulbosus, Vahl. Chilanti-arichi, T.

V.22

2419. conglomeratus, Rottb. var. $\beta$ pachyrhizus, Trim.

2420. arenarius, Retz. Mudu-kalanduru, S. V.23

2421. aristatus, Rottb.

V.24

2422. platystylis, $\mathrm{Br}$.

V.24

2423. difformis, $L$.

V.25

2424. castaneus, Willd.

V.25

2425. cuspidatus, H. B. K.

V.26

2426. Haspan, L. Halpan, S.

V.26

2427. Havidus, Retz.

V.27

2428. pulcherrimus, Willd.

V.27

2429. diffusus, Vahl. var. $\beta$ pubisquama, Hk. $\mathrm{f}$.

V.28

2430. articulatus, $\mathrm{L}$.

V.29

2431. corymbosus, Rottb. Gal-ehi, S.

V.29

2432 dehiscens, Nees Hewan-pan, S.

V.30

2433. distans, L. f.

V.30

2434. nutans, Vahl.

V.31

2435. pilosus, Vaphl.

V.32

2436 exaltatus, Retz.

V.32

var. $\beta$ amcenus, Clarke.

2437. tuberosus, Rottb.

V.33

2438. compressus, L.

V.33

2439. procerus, Rottb.

V.34

2440. Zollingeri, Steud.

V.35

2441. rotundus, L. Kalanduru, S. Korai, T. V.35

2442. stoloniferus, Retz.

V.36

2443. digitatus, Roxb.

var. $\beta$ Hookeri, Clarke.

2444. eleusinoides, Kuntl.

2445. platyphyllus, Rœm. \& Sch.

V.36

2446. alopecuroides, Rottb.

2447. SP. Nov. (Ritigala). ${ }^{1}$

925. Mariscus, Vahl.

2448. Dregeanus, Kunth.

2449. albescens, Gaudich. Ramba, S. Irampai, $\mathrm{T}$.

2450. microcephalus. Presl.

1 Willis, Flora of Ritigala, Ann. Perad., II., p. 289. 
2451. paniceus, Vahl.

var. $\beta$ Roxburghiana, Clarke.

2452. cyperinus, Vahl.

V.42

2453. Sieberianus, Nees.

V.42

2454. tenuifolius, Schrad.

V.43

926. Kyllinga, Rottb.

2455. cylindrica, Nees.

V.44

2456. monocephala, Rottb. Mottu-tana, S. V.44

2457. triceps, Rottb.

V.45

2458. brevifolia, Rottb.

V.45

2459. melanosperma, Nees.

V.45

927. Fimbristylis, Vahl.

2460. tetragona, $\mathrm{Br}$.

2461. acuminata, Vahl.

V.48

2462. nutans, Vahl.

V.4S

V.48

2463. polytrichoides, Vahl.

2464. schœnoides, Vahl.

V.49

V.49

var. $\beta$ bispicata, Trim.

2465. dichotoma, Vahl.

V.50

2466. ASTTIVALIS, Vahl.

V.51

2467. TRIMENI, Hk. f.

V.52

2468. argentea, Vahl.

V.52

2469. ferruginea, Vahl.

$\checkmark .53$

var. $\beta$ tenuissima, Clarke.

2470. diphylla, Vahl.

V.53

var. $\beta$ major, Thw.

var. $\gamma$ ovalis, Hk. $f$.

2471. spathacea, Roth.

V.54

2472. compressa, Boek.

V.55

2473. quinquangularis, Kunth.

V.55

2474. miliacea, Vahl. Mudu-halpan, S.

V.56

var. $\beta$ congesta, Trim.

2475. globulosa, Kunth. Halpan, S.

V.57

2476. insignis, Thw.

V.57

2477. leptoclada, Benth.

2478. asperrima, Boek.

V.58

2479. tristachya, Thw.

V.58

2480. monostachya, Hassk.

2481. pentaptera, Kunth.

V.59

V.59

2482. monticola, Steud.

V.60

2483. cinnamometorum, Kunth.

V. 60

V.61

2484. FULVESCENS, Thw.

2485. nigrobrunnea, Thw.

V.62

2486. complanata, Link.

V.62

V.63

2487. Kraussiana, Hochst.

V.63

2488. junciformis, Kunth. 
928. Echinolytrum, Desv.

2489. dipsaceum, Desv.

929. Bulbostylis, Kunth.

2490. puberula, Kunth.

2491. barbata, Kunth. Uru-hiri, S. var. $\beta$ pulchella, Clarke.

V.66

$\nabla .66$

2492. capillaris, Kunth. var. trifida, Clarke.

V.67

930. Eleocharis, $\mathrm{R} . \mathrm{Br}$.

2493. plantaginea, Br. Boru-pan, S. V.68

2494. equisetina, Presl.

V.69

2495. variegata, Kunth. var. laxiflora, Clarke. V.69

2496. fistulosa, Schultes.

V.70

2497. spiralis, R. Br.

2498. Chætaria, Rœm. \& Sch.

V.70

2499. atropurpurea, Kunth.

V.71

2500. capitata, $R$. Br.

2501. congesta, D. Don.

V.71

2502. tetraquetra, Nees.

V.72

V.72

V.72

931. Scirpus, L.

2503. fluitans, L.

2504. squarrosus, L.

V.73

2505. supinus, L.

V.74

2506. erectus, Poir.

2507. articulatus, L. Maha-geta-pan, S.

V.74

V.75

2508. mucronatus, L.

V.75

2509. subcapitatus, Thw.

V.76

2510. grossus, L. f.

2511. littoralis, Schrad.

V.76

V.77

V.77

932. Websteria, S. H. Wright.

2512. limnophila, S. H. Wright.

V.78

933. Fuirena, Rottb.

2513. glomerata, Lam.

2514. uncinata, Kunth.

2515. umbellata, Rottb.

V.79

V.79

V.80

934. Lipocarpha, $\mathrm{Br}$.

2516. argentea, $\mathrm{Br}$.

2517. triceps, Ness.

V.81

935. Actinoschœnus, Benth.

2518. filiformis, Benth.

V.82

936. Rhyncospora, Vahl.

2519. Wallichiana, Kunth. V.83

2520. aurea, Vahl.

V.83

2521. triflora, Vahl.

V. 84

$6(11) 10$ 
2522. gracillima, Thw.

2523. glauca, Vahl.

var. $\beta$ chinensis, Clarke.

937. Cladium, P. Br.

2524. undulatum, Thw. $\quad$ V.86

2525. riparium, Benth. var. crassum, Clarke. V.87

938. Remirea, Aubl.

2526. maritima, Aubl.

V.87.

939. Lepironia, L. C. Rich.

2527. mucronata, Rich. Eta-pan, S. V.88

940. Hypolytrum, L. C. Rich.

2528. latifolium, Rich.

V.89

var. $\beta$ minus, Thw.

var. $\gamma$ turgidum, Hk. f.

2529. LONGIROSTRE, Thw.

941. Mapania, Aubl.

2530. zeylanica, Benth.

V.91

2531. mimersa, Benth.

V.91

942. Scirpodendron, Zipp.

2532. costatum, Kurz. Hin-keyiya, S. V.92, Pl. XंCVII.

943. Scleria, Berg.

2533. Neesii, Kunth. Baka-munu-tana, S. $\quad$ V.94

2534. pergracilis, Kunth. Mehi-wal, S. V.94

2535. corymbosa, Roxb. V.95

2536. JUNCIFORMIS, Thw. V.95

2537. lithosperma, Sw. $\quad$ V.96

2538. sumatrensis, Retz. $\quad$ V.96

2539. zeylanica, Poir. V.97

2540. elata, Thw. $\quad$ V.97

2541. chinensis, Kunth. var. biauriculata,

Clarke.

V.98

2542. tessellata, Willd. $\quad$ V.98

2543. hebecarpa, Nees. Goda-karawu, S. $\quad$ V.99

2544. biflora, Roxb. $\quad$ V.99

2545. oryzoides, Presl. Potu-pan, Polu-kola, S. V.99

2546. lævis, Retz. V.100

944. Diplacrum, Br.

2547. caricinum, Br.

V.101

945. Carex, L.

2548. nubigena, D. Don.

V.102

2549. brunnea, Thunb.

V.103

2550. longipes, D. Don.

V.103

2551. longicruris, Nees.

V.104

2552. phacota, Spreng.

V.105 
2553. arnottiana, Nees.

2554. rara, Boott.

V.105

2555. Walkeri, Arn.

V.105

2556. SPICIGERA, Nees.

V.106

V.106

var. $\beta$ minor, Thw.

var. $\gamma$ rubella, Clarke.

var. \& rostrata, Bœek.

2557. leucantha, Arn.

V.107

2558. baccans, Nees.

V.107

2559. indica, L. var. latebrunnea, Clarke.

V.108

2560. Lindleyana, Nees.

V.109

2561. ZEXLANICA, Bœck.

V.109

2562. filicina, Nees.

V.110

2563. maculata, Boott.

V.110

2564. BREVISCAPA, Clarke.

2565. hebecarpa, C. A. Mey (ligulata, Nees.)

V.111

2566. Jackiana, Boott.

V.111

var. $\beta$ minor, Clarke.

V.112

2567. lateralis, Kukenth.

2568. LOBULIRostris, Drejer.

V.113

149. Gramineæ.

946. Paspalum, L.

2569. scrobiculatum, L. Amu, S. Waragu, T. V.121

*2570. conjugatum, Berg. ${ }^{1}$

2571. sanguinale, Lamk. Guruwal, S. - V.123

2572. longiflorum, Retz.

2753. Royleanum, Nees.

V.124

2547. Perrottetii, Hk. f.

V.125

V.125

947. Eriochloa, H. B. K.

2575. polystachya, H. B. K.

V.126

948. Isachne, $\mathrm{Br}$.

2576. Kunthiana, W. \& A.

V.127

2577. ELATIOR, Hk. f.

V.127

2578. YULTIFLORA, Trim.

V.127

2579. australis, $\mathrm{R}$. $\mathrm{Br}$.

V.128

var. $\beta$ effusa, 'Trim.

2580. miliacea, Roth.

V.128

2581. Walkeri, W. \& A.

V.129

2582. Gardneri, Benth.

V.130

949. Panicum, L.

*2583. Isachne, Roth.

V.133

2584. flavidum, Retz.

V.133

2585. punctatum, Burm.

V.134

2586. fluitans, Retz.

V.135

1 This and the two next usually placed in Panicum. 
2587. Crus-galli, L. Wel-marukku, S.

var. 3 frumentaceum, Trim.

var. $\gamma$ stagninum, Trim.

2588. colonum, L.

V.136

2589. ambiguum, 'Trin.

V.137

2590. oryzoides, Sw.

V.138

2591. prostratum, Lamk.

V.138

2592. villosum, Lamk.

*2593. muticum, Forsk.

Water grass, Mauritius grass.

V.139

2594 ramosum, L.

2595. setigerum, Retz.

2596. javanicum, Poir.

2597. distachyum, L.

2598. semiverticillatum, Rottl.

2599. remotum, Retz.

2600. canaliculatum, Nees.

2601. nodosum, Kunth.

V.140

V.140

V.141

V.142

V.142

V.143

V.144

V.144

2602. auritum, Presl.

V.145

V.145

2603. Myurus, H. B. K.

V.146

2604. interruptum, Willd.

V. 147

2605. indicum, L.

V.147

var. $\beta$ brachiatum, Hlk. f.

2606. myosuroides, $\mathrm{Br}$.

2607. curvatum, L.

V.148

V.148

2608. ovalifolium, Poir.

V.148

*.2609. miliace:ın. L. Wal-meneri, S. Kadai, Kannai, T.

V.150

*.2610. miliare, Lamk. Meneri, S. Chamai, T. V.150

2611. cæsium, Nees.

V.151

2612. trypheron, Schult. Meneri, S.

V.152

2613. humile, Nees.

V.152

*2614. maximum, Jacq. Rata-tana, S. Gurnea grass.

2615. 1'epens, L. Etora, S.

V.153

V.154

V.155

2616. proliferum, Lam.

V.155

2617. montanum, Roxb.

V.156

2618. antidotale, Retz. Kirimisastru, S.

V.157

. plicatum, Lamk.

V.157

2620. trigonum, Retz.

V.158

2621. pilipes, Nees. \& Arn.

V.159

2622. patens, L.

V.159

2623. SPARSICOMUM, Nees.

V.160

950. Luhnanthus, Beauv.

2625. pallens, Munro. 


\section{$(107)$}

951. Setaria, Beauv.

2626. glanca, Beaur. Kuwulu, S.

V.162

2627. verticillata, Beauv.

V.163

2628. intermedia, Roem. \& Sch.

V.163

2629. gracillima, Hk. f.

V.164

952. Chamæraphis, Br.

2630. spinescens, Poir.

var. $\beta$ aspera, Hk. $f$.

V.165

var. $\gamma$ subglabra, Thw.

var. $\delta$ depauperata, Hk. f.

953. Axonopus, Beauv.

2631. cimicinus, Beauv.

2632. semialatus, Hk. f.

V.166

V.167

954. Oplismenus, Beauv.

2633. compositus, Beauv.

V.168

2634. Burmannii, Beauv.

V.169

2635. THWAITESIr, Hk. f.

V.169

955. Pennisetum, Pers.

*2636. typhoideun, Rich. Kumba, 'T. Bulrush millet.

2637. orientale, Rich.

V.171

956. Stenotaphrum, Trin.

2638. complanatum, Schrank.

957. Thuarea, Pers.

2639. salmentosa, Per's.

V.173

958. Spinifex, L.

2640. squarrosus, L. Maha-rauunc-rewult, S. V.17t

959. Arundinella, Raddi.

2641. avenacea, Munro.

V.176

var. $\beta$ robusta, Hk. f.

2642. setosa, Trin.

V.177

2643. villosa, Arn.

V.178

2644. leptochloa, Hk. f.

V.178

2645. LAXIFLORA, Hk. f.

V.179

2646. Lawii, Hk. f.

V.180

2647. BLEPHARIPHYLLA, Trim.

V.180

2648. THWAITESII, Hk. f.

V.181

960. Oryza, L.

2649. sativa, L. Uru-wi, S. Wild paddy. $\quad$ V.182

2650. granulata, Nees.

V.183

2651. latifolia, Desv.

V:184

961. Leersia, Sw.

2652. hexandra, Sw. Layu, S. 
962. Hygrorhiza, Nees.

2653. aristata, Nees. Go-jabba, s.

V.185

963. Trachys, Pers.

2654. mucronata, Pers.

V.186

964. Tragus, Haller.

2655. racemosus, Scop.

V.187

965. Zoysia, Willd.

2656. pungens, Willd.

V.188

966. Lopholepis, Dene.

2657. ornithocephala, Dene.

V.189

967. Peretis, Ait.

2658. latifolia, Ait.

$\dot{\mathrm{V}} .189$

968. Leptaspis, Br.

2659. urceolata, $\mathrm{Br}$.

2660. COCHLEATA, Thw.

V.190

V.191

969. Coix, L.

2661. Lachryma-jobi, L. Kikirindi, S.

V.192

970. Polytoca, $\mathrm{Br}$.

2662. barbata, Stapf.

V.194

971. Dimeria, Br.

2663. pusilla, Thw.

var. $\beta$ elatior, Hk. $f$.

2664. PUBESCENS, Hack.

2665. Lehmanni, Hack.

var. $\alpha$ aristata, Hack.

var. $\beta$ mutica, Hack.

2666. THWAITESח, Hack.

2667. fuscescens, Trin. var. $\beta$ robusta, Hk. $f$.

2668. TRLMENI, Hk. f.

2669. gracilis, Nees.

V.195

V.196

V.196

V.197

V.198

V.198

V.199

972. Imperata, Cyrill.

2670. arundinacea, Cyrill. $1 l u k, \mathrm{~S}$.

V.200

973. Saccharum, L.

2671. spontaneum, L.

2672. arundinaceum, Retz. Rambuk, S. Peykarumu, T.

V.201

V.202

974. Pollinia, Trin.

2673. THWAItesII, Hack.

2674. argentea, Trin.

V.203

2675. phæothrix, Hack.

V.204

2676. ciliata, Trin.

V.204

V.205 
975. Rottbœllia, L. f.

2677. compressa, L. f.

2678. exaltata, I. f.

2679. NIGRESCENS, Thw.

V.206

V.207

V.207

976. Manisuris, $\mathrm{Sw}$.

2680. granularis, L. f.

V.209

977. Mnesithea, Kunth.

2681. lævis, Kunth.

V.210

978. Ischæmum, L.

2682. aristatum, L. var. $\beta$ fallax, Hack.

V.211

2683. rugosum, Salish.

*2684. semisagittatum, Roxb.

V.212

2685. commutatum, Hack.

2686. muticum, L.

2687. ciliare, Retz. Rut-tana, S.

V.213

V.214

V.215

2688. RIVALE, Hack.

2689. timorense, Kunth.

2690. laxum, Br.

$\cdot V .216$

V.217

V.218

V.219

979. Eremochloa, Buse.

2691. muricata, Hack.

2692. ZEYLANICA, Hack.

V.220

V.221

980. Pogonatherum, Beauv.

2693. crinitum, Kunth.

V.222

981. Apocopis, Nees.

2694. Wightii, Nees.

var. $\alpha$ genuinus, Hack.

var. $\beta$ mangalurensis, Hack.

982. Arthraxon, Beauv.

2695. rudis, Hochst.

2696. microphyllus, Hochst.

V.224 2697. ciliaris, Beauv.

V.223

V.224

V.225

983. Apluda, L.

2698. varia, Hack.

V.226

984. Andropogon, L. ${ }^{1}$

2699. Pseudischæmum, Nees.

V.229

2700. pertusus, Willd.

V.230

2701. intermedius, $\mathrm{Br}$.

V.230

2702. halepensis, Brot.

V.231

2703. serratus, Thunb.

V.232

2704. aciculatus, Retz. Tuttiri, S. Love gruss. V.234

2705. zeylanicus, Nees.

2706. monticola, Schult.

V.235

2707. caricosus, L.

V.236

V.237

${ }^{1}$ Cf. Stapf in Kew Bulletin, 1906, p. 297. 
2708. polyptychus, Steud.

2709. contortus, L. Itana, S. V.238

2710. triticeus, Br.

V.239

2711. hirtiflorus, Kunth.

V.240

985. Vetiveria, Thou. ${ }^{1}$

2712. zizanioides, Stapf. (Andropogon squarrosus, L. f.). Scewandara, S. Vetti-ver, T. Khas-khas.

2713. venustus (Thw.), Willis.

V.233

986. Cymbopogon, Spreng. ${ }^{1}$

2714. Schœenanthus, Spreng (A. Schœenanthus, L.). V.24I 2715. Nardus, Rendle (A. Nardus, L.). Lenabatu, S. Citronella grass.

2716. Winterianus, Jowitt. Maha-pengiri, S.

V.242

Winter's citronella grass

2717. confertiflorus, Stapf. Mana, S.

2718. citratus, Stapf. Lemon grass.

2719. polyneuros, Stapf.

2720. THWAITESII (Hk. f.), Willis.

V.243

2721. lividus (Thw.). Willis.

V.244

2722. filipendulus (Hochst.), Willis.

V.245

987. Pseudanthistiria, Hk. f.

2723. umbellata, Hk. f.

V.247

988. Anthistiria, L.

2724. imberbis, Retz.

V.248

2725. cymbaria, Roxb. Karawata-mana, S.

V.249

2726. tremula, Nees. Pini-buru-tana, S.

V.249 var. $\beta$ Thwaitesii, Hk. f.

var. $\gamma$ brunnea, Hk. $f$.

989. Iseilema, Anderss.

2727. laxum, Hack.

V.251

990. Aristida, L.

2728. Adscensionis, L. Teli-tana, S.

V.252

2729. setacea, Retz. Et-tuttiri, S.

V.253

991. Garnotia, Brongn.

2730. THWAITESII, Stapf.

2731. TECTORUM, Hk. f.

V.254

V.254

2732. FUSCATA, Thw.

V.255

2733. Fergusonit, Trim.

V.255

var. $\beta$ fastigiata, Hk. f.

2734. MICRANTHA, Thw.

V.256

2735. courtallensis, Thw.

2736. Panicoides, Trim. 
992. Sphærocaryum, Nees.

2737. elegans, Nees.

V.258

993. Polypogon, Desf.

2738 . monspeliensis, Desf.

V.259

994. Sporobolus; Br.

2739. diander, Beauv. var. $\beta$ nanus, Hk. $f$.

V.260

2740. indicus, $\mathrm{Br}$.

V.261

2741. Wallichii, Munro.

V.261

2742. virginicus, Kunth. Mudu-etora, S. V.262

2743. tremulus, Kuntl.

V.263

2744. orientalis, Kunth.

V.263

2745. coromandelianus, Kunth.

V.264

995. Calamagrostis, Adans.

2746. pilosula, Hk. f.

V.264

996. Avena, L.

2747. aspera, Munro.

V.265

997. Eriachne, Br.

2748. triseta, Nees. Pini-tuttiri, S.

V.266

998. Zenkeria, Trin.

2749. OBTUSIFLoRA, Benth.

V.267

2750. elegans, Trin.

V.268

999. C'œlachne, Br.

2751. pulchella, Br. var. simpliciuscula, Hk. f. V.269

2752. PERPUSILLA, Thw.

V.270

var. $\beta$ muscosa, Hk. f.

100c. Oropetium, Trin.

2753. Thomæum, Trin.

1601. Enteropogon, Nees.

2754. melicoides, Nees.

V.272

1002. Tripogon, Roth.

2755. bromoides, Rotli.

V.273

1003. Cynodon, Pers.

2756. Dactylon, Pers. Arugam-pillu, 'T. Bermuda grass, Doob grass.

1004. ('hloris, Sw.

2757. incompleta, Roth.

V.275

2758. barluata, Sw. Mayuru-tana, S.

V.275

2759. montana, Roxb.

V.276

$6(11) 10$ 
1005. Eleusine, Gærtn.

2760. indica, Gærtn. Wal-kurakkan, S. V.277

2761. verticillata, Roxb.

V.277

2762. brevifolia, $\mathrm{Br}$.

V.278

2763. ægyptiaca, Desf.

V.279

1006. Dinebra, Jacq.

*2764. arabica, Jacq.

V. 280

1007. Dichretaria, Steud.

2765. Wightii, Nees.

V.281

1008. Leptochloa, Beauv.

2766. uniflora, Hochst.

2767. polystachya, Benth.

V.282

V.282

2768. filiformis, Beauv.

V.283

2769. chinensis, Nees.

V.283

1009. Gracilea, Kœn.

2770. nutans, Kœn.

V.284

1010. Pommereulla, L. f.

2771. Cornucopire, L. f.

V.256

1011. Phragmites, Trin.

2772. Karka, Trin. Nala-gas, S.

V.287

1012. Elytrophorus, Beauv.

2773. articulatus, Beauv.

V.288

1013. Myriostachya, Hk. f.

2774. Wightiana, Hk. f.

V.288

1014. Eragrostis, Host.

2775. tenella, Rœm. \& Sch.

var. $\beta$ plumosa, Stapf.

var. $\gamma$ contracta, Hk. f.

var. $\delta$ riparia, Stapf.

var. $\varepsilon$ viscosa, Stapf.

var. $\varsigma$ densiflora, Hk. f.

2776. interrupta, Beauv.

var. $\beta$ diplachnoides, Stapf.

V.292

var. $\gamma$ Kœnigii, Stapf.

var. $\delta$ tenuissima, Stapf.

2777. amabilis, W. \& A.

V.290

2778. gangetica, Steud. Ela-kuru-tana, S.

V.293

2779. stenophylla, Hochst.

V.293

V.294

2780. elongata, Jacq. Mal-etora-trna, S. V.295

2781. nigra, Nees.

V.295

2782. pilosa, Beauv.

V.296

2783. Willdenoviana, Nees. 
2784. major, Host.

2785. coromandeliana, Trin.

2786. SECUNdA, Nees.

V.298

2787. WALKERI, Stapf.

V.298

V.298

1015. Halopyrum, Stapf.

2788. mucronatum, Stapf.

V.299

1016. Diplachne, Beauv.

2789. fusca, Beauv.

V.300

1017. Streptogyne, Beauv.

2790. gerontogæa, Hk. f.

V.301

1018. Lophatherum, Brongn.

2791. gracile, Brongn.

2792. ZEYLANICUM, Hk. f.

V.302

V.303

1019. Centotheca, Desv.

2793. lappacea, Desv.

V.304

1020. Eluropus, Trin.

2794. villosus, 'I'rin.

V.304

1021. Poa, L.

*2795. annua, L.

V.305

1022. Brachypodium, Beauv.

2796. sylvaticum, Beauv.

V.306

1023. Lepturus, Br.

2797. repens, $\mathrm{Br}$.

V.307

1024. Arundinaria, Mich.

2798. Walkeriana, Munro.

V.309

2799. Wightiana, Nees.

V.309

2800. FLORIBUNDA, Thw.

V.310

2801. DEBILIS, Thw.

V.311

2802. densifolia, Munro.

V.312

1025. Bambusa, Schreb.

2803. arundinacea, Willd. Katu-una, S. Moongil, T. Spiny bamboo.

2804. vulgaris, Schrad. Una, S. Bamboo.

V.313

*2805. nana, Roxb. Dwarf or Chinese bamboo.

V.314

V.315

1026. Oxytenanthera, Munio.

2806. Thwaitesii, Munro.

V.316

1027. Teinostachyum, Munro.

2807. atTenUatum, Munro.

V.317

1028. Ochlandra, Thw.

2808. STRIDULA, Thw. Bata-li, S. 


\section{$114)$ \\ VASCULAR CRYPTOGAMS.}

150. Lycopodiaceæ. ${ }^{1}$

1029. Lycopodium, L.

2809. Hamiltonii, Spreng. 10

2810. CEYLANICUM, Spreng. 11

2811. serratum, Thunb. 12

2812. setaceum, Ham. 14

2813. squarrosum, Forst. (Hookeri, Wall.)

Kuda-hedaya, S. $\quad 18$

2814. Phlegmaria, L. Maha-hedaya, S. 22

2815. Phyllanthum, Hook. \& Ain. 22

2816. cernuum, L. Wanassa, Badal-wanassa, S. 23

2817. elavatum, L. $\quad 26$

2818. carolinianum, L. 28

2819. Wightianum, Wall. 28

2820. complanatum, L. 28

150. Psilotaceae. ${ }^{1}$

1030. Psilotum, Sw.

2821. triquetrum, Sw.

152. Selaginellaceæ. ${ }^{1}$

1031. Selaginella, Spring.

2822. rupestris, Spring.

2823. plumosa, Baker. 50

2824. INTEGERRIMA, Spring. $\quad 66$

2825. cochleata, Spring. $\quad 76$

2826. caulescens, Spring. 94

2827. latifolia, Spring. 98

2828. CILIARIS, Spring. (?) 105

2829. proniflora, Baker. 108

2830. ZEYLANICA, Baker. 111

2831. brachystachya, Spring. 113

2832. CRASSIPES, Spring. 117

2833. tenera, Spring. 118

153. Isœtaceæ. ${ }^{1}$

1032. Isoetes, L.

2834. coromandelina, L. f.

132

154. Equisetaceæ. ${ }^{1}$

1033. Equisetum, L.

2835. debile, Roxb.

1 Following Raker, Handbook of tho Forn-allies, to which the pagos quoted refer. 
155. Salviniaceæ. ${ }^{1}$

1034. Azolla, Lam.

2836. pinnata, R. Br.

138

156. Marsileaceæ. ${ }^{1}$

1035. Marsilea, L.

2837. quadrifolia, L. Diya-embul-embiliya, S. Ara-kiri, T.

2838. minuta, L.

\section{Hymenophyllaceæ. ${ }^{2}$}

1036. Trichomanes, L.

2839. Motleyi, v. d. B.

2840. WALLII, Thw.

2841. exiguum, Baker.

2842. bimarginatum, v. d. B. (neilgherrense,

Bedd.)

2843. parvulum, Poir.

2844. proliferum, Bl.

2845. digitatum, Sw.

2846. intramarginale, Hk. \& Grev.

2847. pallidum, Bl.

2848. bipunctatum, Poir.

2849. pyxidiferum, L.

2850. rigidum, $\mathrm{Sw}$.

2851. sp. nov. (Ritigala). ${ }^{3}$

1037. Hymenophyllum, Sm. 2852. tenellum, Kuhn.

2853. exsertuin, Wall.

2854. polyanthos, Sw.

2855. Blumeanum, Spreng.

2856. australe, Willd. (javanicum, Spreng.)

2857. aculeatum, Racib. (Neesii, Hk. p. p.)

\section{Cyatheaceæ.}

1038. Cyathea, Sm.

2858. SINUATA, Hk. \& Grev.

2859. HOOKERT, Thw.

1 Following Baker, Handbook of the Fern-allies, to which the pages quoted refer.

2 The ferns are arranged according to Christensen's Index Filicum : tho number reforonces are to tho pages of Boddomo's Handbook of the Forns of British India, Coylon, \&c.

3 Willis, Flora of Ritigala, Ann. Porad., 1II., 1906, p. 290. 


\section{$(116)$}

1039. Hemitelia, R. Br.

2860. WALKËR Pr. (Amphicosmia Walkeræ, Moore.)

1040. Alsophila, R. Br. 2861. glabra, Hk. 2862. crinita, Hk.

\section{Polypodiaceæ.}

1041. Diacalpe, Bl.

2863. aspidioides, $\mathrm{Bl}$.

1042. Dryopteris, Adans. (Nephrodium, Lastrea, \&c.) § 1. Eudryopteris (Lastrea auct.)

2864. hirtipes (Bl.) O. Ktze.

2865. WALKer (Hk.), O. Ktze.

232

var. $\beta$ macrocarpa, Bedd.

233

var. $\gamma$ pinnatifida, Bedd.

var. $\delta$ bipinnata, Bedd.

¿866. calcarata (BI.), O. Ktze.

var. $\beta$ Moonii, Trim. Ms.

2867. Beddomei (Bak.), O. Ktze.

239

2868. ochthodes (Kze.), C. Chr.

240

2869. syrmatica (Willd.), O. Ktze.

243

2870. flaccida (Bl.), O. Ktze.

244

2871. filixmas (L.), Schott.

248

var. $\beta$ elongata (Pr.), C. Chr.

2872. sparsa (Ham.), O. Ktze.

var. $\beta$ obtusissima (Mett.), C. Chr.

var. $\gamma$ deltoidea (Bedd.), C. Chr.

var, o minor (Bedd.), C. Chr.

var. \& zeylanica (Bedd.), C. Chr.

var. $\varsigma$ undulata (Thw.), C. Chr.

2873. deparioides (Moore), O. Ktze.

var. $\beta$ concinnum (Thw.), C. Chr. (L.

Thwaitesii, Bedd.)

2874. crenata (Forsk.), O. Ktze.

258

2875. rhodolepis (Clarke), C. Chr. (L. Blumei, Moore.)

2876. ricedens (J. Sm.), O. Ktze.

2877. dissecta (Forst.), O. Ktze.

2878. peranemiformis, C. Chr.

$$
\text { var. } \beta \text { obtusilobum (Bak.), O. Ktze. }
$$

2879. Boryana (Willd.), C. Chr.

2880. setigera (Bl.), O. Ktze. (L. tenericaulis,

Moore.)

§ 2. Phegopteris.

2881. brunnea (Wall.), C. Chr. (P. distans, Mett.) 292 
2883. rufescens (BI.), C. Chr.

2883. punctata (Thunb.), C. Clır.

2884. prolifera (Retz.), C. Chr. (Goniopteris prolifera, Bedd.)

§ 3. Leptogramma.

2885. afiricana (Desv.), C. Chr. (Leptogramma lotta, J. Sm.)

$\S 4$. Cyclosorus. (Nephrodium, Beddome.)

2886. Otaria (Kze.), O. Ktze.

2887. gongylodes (Schk.), O. Ktze. (N. unitum,

R. Br.)

2888. pteroides (Retz.), O. Ktze.

268

2889. extensa (B1.), O. Ktze.

2890. unita (L.), O. Ktze. (N. cucullatum, Bak.) 270

2891. urophylla (Wall.), C. Chr.

2892. arbuscula (Willd.), O. Ktze.

2893. megaphylla (Mett.), C. Chr. (N. pennigerum,

Moore.)

274

276

289t. parastica (I.), (1. Ktze. (N. molle, R. Br.) 277

2894. parasitica (L.), O. Ktze. (N. molle, R. Br.) 277 var. $\beta$ amboinense (Willd.), O. Ktze.

2895. truncata (Poir.), O. Kitze.

$\$ 6$. Stegnogranma.

2896.? [stegnogramme (Bl.), C. Chr. (Stegnogramme aspidioides, Bl.)

$\S 7$. Meniscium.

2897. triphylla (Sw.), C. Chr.

2898. Thwaitesii (Hk.), C. Chr.

1043. Aspidium, Sw.

2899. devexum, Kze. (Pleosnemia membranarea,

Bedd., A. membranaceum, Hk.)

2900. subtriphyllum, Hk.

2901. polymorphum, Wall.

2902. decurrens, Presl.

var. $\beta$ minor, Bedd.

2903. cicutarium, Sw.

2904. ThwaItesII, Bedd. (Pleoenemia Thwait-

220 esii, Bedd.)

2905. giganteum, Bl. (Pleocnemia Trimeni, Bedd.)

1044. Polystichum, Roth.

2906. auriculatum (T.), Pr.

2907. aculeatum (L.), Schott.

var. $\beta$ angulare, Presl. (sp.)

var. $\gamma$ biaristatum. Moore (sp.)

var. $\delta$ a nomalum, Hk. \& Arn. (sp.) 
2908. amabile (B1.), J. Sin. (Lastrea amahilis, Moore.)

2909. aristatum (Forst.), Pr. (Lastrea aristata, Moore.)

2910. carvifolium (Kze.), C. Chr. (Lastrea coniifolia, Moore, Polystichum coniifolium, Pr.)

1045. Polybotrya, Humb. \& Borpl.

2911. appendiculata (Willd.), J. Sm.

424

1046. Leptochilus, Kaulf.

2912. decurrens, BI. (Gymmopteris variabilis,

Bedd.)

$4 \cdot 29$

2913. lanceolatus, Fée (vas. in Becldome).

429

2914. Wallii (Bak.), C. Chr. (G. Wallii, Bedd.)

431

2915. ietallicus (Bedd.), C. Chr. (G. metallica, Bedd.)

2916. zeylanicus (Houtt.), C. Chr. (G. quercifolia, Bedd.)

432

2917. virens (Wall.), C. Chr. (G. contaminans, Bedd.)

432

2918. subcrenatus (Hk. \& Grev.), C. Chr. (G. subcrenata, Bedd.)

435

437

1047. Oleandra, Cavanilles.

2919. musifolia (Bl.), $\mathrm{Pr}$.

1048. Arthropteris, J. Sm.

2920. obliterata (R. Br.), J. Sm. (Nephrolepis ramosa, Moore.)

1049. Nephrolepis, Schott.

2921. cordifolia (L.), Pr.

2922. exaltata (L.), Schott.

282

2923. biserrata (Sw.), Schott. (N. acuta, Pr.) 284

1050. Humata, Cavanilles.

2924. repens (L. f.), Diels. (H. pedata, J. Sm.) 48

2925. vestita (Bl.), Moore. 48

1051. Davallia, Sm.

2926. pulchra, Don. (Leucostegia pulchra, J. Sm.) 52 2927. hymenophylloides (Bl.), Kuhn. (Leucostegia hymenophylloides, Bedd.) 2928. alata, Bl. (Prosaptia Emersoni, Pr.) 56 2929. contigua (Forst.), Spr. (Prosaptia contigua,

Pr.)

2930. denticulata (Burm.), Mett. (D). elegans, Sw.) 5 ? 2931. bullata, Wall. 
1052. Microlepia, Pr.

2932. platyphylla (Don), J. Sm. 66

2933. MaJUSCULA (Lowe), Moore. 66

2934. strigosa (Thunb.), Pr. 67

2935. speluncæ (L.), Moore. $\quad 67$

2936. hirta (Kaulf.), Pr. 68

1053. Odontosoria (Presl.), Fée.

2937. chinensis (L.), J. Sm. (Stenoloma chinensis, Bedd.)

1054. Dennstædtia, Bernhardi.

2938. scabra (Wall.), Moore.

1055. Schizoloma, Gaudich.

2939. Walkeræ (Hk.), Kuhn. (Lindsaya Walkeræ, Hk.)

2940. ensifolium (Sw.), J. Sm.

2941. heterophyllum (Dry.), J. Sm.

1056. Lindsaya, Dryand.

2942. cultrata (Willd.), Sw.

2943. repens (Bory), Bedd.

72 var. $\beta$ minor, Thw.

2944. orbiculata (Lam.), Mett.

var. $\beta$ tenera, Bedd.

var. $\gamma$ schizophylla (Baker).

2945. lancea (L.), Bedd.

2946. decomposita, Willd. (Schizoloma lobata,

Bedd.)

1057. Athyrium, Roth.

2947. Hohenackerianum (Kze.), Moore.

163

2948. macrocarpum (Bl.), Bedd.

2949. solenopteris (Kze.), Moore.

166

2950. GYMNogrammoides (Kl.), Bedd. var. $\beta$ erythrorachis, Bedd.

168

2951. assimile (Endl.), Pr. (Diplazium umbrosum,

Bedd., var. assimile, Bedd.)

1058. Diplazium, Sw.

2952. lanceum (Thunb.), Pr.

174

2953. zeylanicum (Hk.), Moore.

2954. silvaticum (Bory.), Sw.

175

2955. japonicum (Thunb.), Bedd.

177

var. $\beta$ Thwaitesii, Kl. (sp.)

180

2956. BEDDONEı, C. Chr. (D. Schkuhrii, Bedd.) 181

2957. polypodioides, $\mathrm{Bl}$.

184

var. $\beta$ decurrens, Bedd.

2958. maximum (Don), C. Chr. (D) latifolium,

Moore.) 
2959. esculentum (Retz.), Sw. (Anisogonium

esculentum, Pr.) Miwana-kola, S.

2960. Smithianum (Bak.), Diels. (Anisogonium

Smithianum, Bedd.)

1059. Diplaziopsis, C. Chr.

2961. javanica (Bl.), C. Chr. (Allantodia javanica, Trevis.)

1060. Asplenium, L.

2962. nidus, L. (Thamnopteris Nidus, Pr.) 137

2963. ensiforme, Wall. 141

2964. normale, Don. 144

2965. Wightianum, Wall. 146

2966. vulcanicum, Bl. 146

2967. tenerum, Forst. 147

2968. lunulatum, Sw. $\quad 147$

var. $\beta$ camptorachis, Kze.

2969. Zenkerianum, Kze.

2970. adiantoides (L.), C. Chr. (A. falcatum, Lam.)

2971. macrophyllum, Sw.

2972. caudatum, Forst.

2973. Gardneri, Bak.

2974. formosum, Willd.

2975. unilaterale, Lam.

152

2976. cheilosorum, Kze. (A. heterocarpum,Wall.) 153

2977. laciniatum, Don.

154

var. $\beta$ planicaule, Wall. (sp.)

2978. præmorsum, Sw. (A. furcatum, Thunb.) 157

2979. affine, Sw.

2980. nitidum, Sw.

2981. varians, Wall.

2982. tenuifolium, Don.

2983. achilleifolium (Lam.), C. Chr. (A. rutæfolium, Kze.)

1061. Blechnum, L.

2984. orientale, $\mathrm{L}$.

2985. Patersoni (R. Br.), Mett. (Lomaria Patersoni, sp.)

1062. Stenochlæna, J. Sm.

2986. palustris (Burm.), Bedd. Wel-benduru, S.

2987. aculeata (Bl.), Kze. (S. palustris, var. achilleifolia, Wall. (sp.)

1063. Doodia, R. Br.

2988. dives, Kze. 
1064. Anogramma, Link.

2989. leptophylla (L.), Link.

1065. Coniogramme, Fée.

2990. fraxinea (Don), Diels. (Syngramme fraxinea, Bedd.)

386

1066. Hemionitis, L.

2991. arifolia (Burm.), Moore.

413

1067. Pellæa, Link.

2992. Boivini, Hk.

2993. falcata (R. Br.), Fée.

1068. Doryopteris, J. Sm.

2994. concolor (Langsd. and Fisch.), Kuhn. (Pellæa concolor, Bak.)

1069. Cheilanthes, Sw.

2995. mysurensis, Wall.

2996. Thwaitesii, Mett. (C. laxa, Moore.)

2997. tenuifolia (Burm.), Sw.

2998. farinosa (Forsk.), Kaulf.

1070. Adiantum, L.

2999. lunulatum, Burm.

82

3000. caudatum, $\mathrm{L}$.

83

3001. capillus-veneris, L $\quad 84$

3002. hispidulum, Sw.

3003. flabellulatum, L.

86

88

1071. Actiniopteris, Link.

3004. australis (L. f.), Link. (A. dichotoma, Kuhn.)

1072. Pteris, L.

3005. longifolia, $\mathrm{L}$.

106

3006. cretica, L.

106

3007. hookeriana, Agardh.

3008. ensiformis, Burm.

107

3009. decussata, J. Sm.

(P. patens, Hk.)

107

3010. longipes, Don.

114

3011. biaurita, L. (Pteris quadriaurita, Retz.,

Campteria biaurita, Hk.)

115 var. $\beta$ ludens, Thw.

3012. tripartita, Sw. (Litobrochia marginata, Pr.) 122

Histiopteris (Agardh.), J. Sm.

3013. incisa (Thunb.), J. Sm. (Litobrochia incisa, Pr.)

1074. Pteridium, Gleditsch.

3014. aquilinum (L.), Kuhn. (Pteris aquilina, L.) Bracken. 
1075. Monogramma, Schkuhr.

3015. paradoxa (Fée.), Bedd.

1076. Vittaria, J. Sm.

3016. elongata, Sw. 404

3017. flexuosa, Fée. (V. lineata, Bedd.) 407

3018. scolopendrina (Bory), Thw. 408

3019. sulcata (Mett.), Kuhn. 409

1077. Antrophyum, Kaulf.

3020. reticulatum (Forst.), Kaulf. 401

3021. plantaginoum (Cav.), Kaulf. 403

1078. Drymoglossum, Pr.

3022. heterophyllum (L.), C. Chr. (D. piloselloides, Pr.)

1079. Tænitis, Willd.

3023. blechnoides (Willd.), Sw.

1080. Hymenolepis, Kaulf.

3024. spicata (L. f.), Pr. (Gymnopteris spicata, Pr.) 432

1081. Polypodium, L.

$\S$ 1. Eupolypodium.

3025. mediale, Bak. (P. parasiticum, Mett.) 302

3026. Ze YLaNicum (Fée.), Mett. 303

3027. hirtellum, Bl.

3028. WALLII, Bedd.

305 .

3029. CoRNIGERUM, Bak.

305

3030. cucullatum, Nees. and Bl.

307

3031. CoRTiColUM, C. Chr. (P. glandulosum,

Hk.)

307

3032. thwaitesu, Bedd.

3033. decorum, Brack.

309

309

3034. obliquatum, $\mathrm{Bl}$.

310

3035. repandulum (Kze.), Mett.

311

3036. subfalcatum, Bl.

313

314

$$
\text { §. Pleopeltis. }
$$

3037. lineare, Thunb.

3038. lanceolatum, $\mathrm{L}$.

351

3039. membranaceum, Don.

355

3040. punctatum (L.), Sw.

357

3041. pteropus, Bl.

3042. hastatum, Thunb.

362

3043. phymatodes, L.

3044. nigrescens, $\mathrm{Bl}$.

3045. euryphyllum, C. Chr. (Pleopeltis dilatata, Bedd.) 
§ 8. Loxogramme.

3046. loxogramme, Mett. (Loxogramme lanceolata, Pr.)

3047. scolopendrinum (Bory.), C. Chr. (L. involuta, Pr.)

1082. Cyclophorus, Desv. (Niphobolus, Kaulf.)

3048. adnascens (Sw.), Desv. (N. lanceolatus, Kөys.)

3049. CEYlanicus (Giesn.), C. Chr.

3050. pannosus (Mett.), C. Chr.

3051. acrostichoides (Forst.), Pr. (N. fissus, BI.)

328

3052. Gardneri (Kze.), C. Chr.

330

331

1083. Drynaria (Bory), J. Sm.

3053. quercifolia (L.), J. Sm. (Pleopeltis quercifolia, Trim.)

3054. sparsisora (Desv.), Moore. (D. Linnæi, Bedd.)

1084. Elaphoglossum, Schott.

3055. conforme (Sw.), Schott.

3056. laurifolium (Thou.), Moore.

3057. hirtum (Sw.), C. Chr. (squamosum, J. Sm.) 3058. spathulatum (Bory), Moore.

1085. Acrostichum, L.

3059. aureum, L. Kere-koku, S.

160. Parkerlaceæ.

1086. Ceratopteris, Brongn.

3060. thalictroides (L.), Brongn.

\section{Gleicheniaceæ.}

1087. Gleichenia, Sm.

3061. linearis (Burm.), Clarke. (G. dichotoma, Hk.) Kekilla, S.

162. Schizæaceæ.

1088. Schizæa, Sm.

3062. digitata (L.), Sw.

452

1089. Lygodium, Sw.

3063. circinatum (Burm.), Sw. (L. dichotomum,

Sw.) Et-pamba, S.

3064. scandens (L.), Sw. (L. microphyllum,

R. Br.) Maha-pamba, S.

3065. flexuosum (L.), Sw. Hin-pamba, S. 
163. Osmundaceæ.

1090. Osmunda, L.

3066. javanica, BI.

164. Marattiaceæ.

1091. Angiopteris, Hoffm.

3067. evecta (Forst.), Hoffm. $\quad 460$

1092. Marattia, Sw.

3068. fraxinea, $\mathrm{Sm}$.

460

165. Ophioglossaceæ.

1093. Ophioglossum, L.

3069. pedunculosum, Desv. (reticulatum, auct.) 465

3070. gramineum, Willd. (O. lusitanicum, A.

$\mathrm{Br}$.)

3071. pendulum, L.

1094. Botrychium, Sw.

3072. daucifolium, Wall.

3073. virginianum (L.), Sw.

1095. Helminthostachys, Kaulf.

3074. zeylanica (L.), Hk.

\section{A Catalogue of the Chief Introduced and Naturalized Species found in Ceylon.}

To complete the preceding catalogue, we have made the following, in which we have included all the species we know to be regularly cultivated in the Colony, or to have escaped and established themselves as weeds therein. Its length will surprise many people, as also the fact that it includes nearly all of our useful plants.

It is very difficult to make a list like th's accurate or complete. New weeds may any day make their appearance, or old ones may disappear, and new cultivations may begin. We have excluded the purely decorative garden plants, unless, as in the case of Cosmos, they have spread into the country and established themselves, or are used in hedges or in similar ways. 
The orders are numbered as in Durand's Index Generum Phanerogamorum, and marked with an asterisk to indicate that they belong to the Supplement. Page references, when given, refer to Trimen's Flora.

\section{I C O T Y LED O N S.}

POLY PETALA.

\section{4.* Magnoliaceæ.}

1. Michelia, L.

†1. Champaca, L. Sapu, Champak. India. I.15

\section{5.* Anonaceæ.}

2. Uvaria, L.

$\dagger 2$. purpurea, Bl. Java.

3. Cananga, Rumph.

†3. odorata, Hk. f. \& Th. Wana-sapu, S. Ylang-ylang. Macassar oil. Burma, Java.

4. Unona, L.

$\uparrow 4$. discolor, Vahl. Indo-Malaya.

5. Anona, L.

†5. muricata, Dun. Katu-anoda, S. Sitha, T. Soursop. Trop. America.

†. reticulata, L. Anoda, S. Ramsitha, T. Bullock's heart. Trop. America.

†7. squamosa, L. Sweet-sop, Sugar apple, Custard apple. E. Indies.

†8. Cherimolia, Mill. Cherimoyer. Trop. America.

10.* Papaveraceæ.

6. Argemone, L.

9. mexicana, L. Trop. America.

7. Bocconia, L.

10. cordata, Willd. China, Japan.

12.* Cruciferæ.

8. Nasturtium, R. Br.

11. officinale, R. Br. Watercress. Europe. 
9. Cochlearia, L.

†12. Armoracia, L. Horse-radish. Europe.

10. Brassica, I.

13. juncea, Hk. f. \& Th. $A b a$, S. India, \&c. I.54

†14. oleracea, L. Cabbage. Europe.

11. Capsella, Medic.

15. Bursa-pastoris, Medic. Shepherd's purse.

N. temp. zone.

I.54

12. Raphanus, $\mathrm{L}$.

$\dagger$ 16. sativus, L. Rabu, S. Radish. Europe.

\section{3.* Capparidaceæ.}

13. Gynandropsis, DC.

17. speciosa, DC. Trop. America.

16.* Violaceæ.

14. Viola, L.

$\dagger 18$. odorata, L. Sweet violet. Europe.

18.* Bixaceæ.

15. Cochlospermum, Kunth.

19. Gossypium, DC. Kinihiriya, Ela-imbul, S. Kongu, T. India.

16. Bixa, L.

†20. Orellana, L. Kaha, S. Annatto, Arnatto, Roucou. Trop. America.

1\%. Flacourtia, Comm.

†21. inermis, Roxb. Lovi-lovi. Malaya.

†22. Cataphracta, Roxb. Rata-uguressa, S. Malaya.

24.* Caryophyllaceæ.

18. Stellaria, L.

23. media, Cyrill. Chickweed. Europe.

19. Sagina, L.

24. procumbens, L. Pearlwort. N. temp. zone.

20. Spergula, L.

25. arvensis, L. Spurrey. N. temp. zone.

25.* Portulacaceæ.

21. Talinum, Adans.

26. patens, Willd. Trop. America.

$\uparrow$ Cultivated only. 


\section{8.* Hypericaceæ.}

22. Hypericum, L.

27. humifusum, L. N. temp. zone.

\section{9.* Guttiferæ.}

23. Garcinia, L.

†28. Mangostana, L. Mangu.s, S. and T. Mangosteen. Malaya.

†29. Xanthochymus, Hk. f. Rata-goraka, S. Seemai-goraka, T. Cochin goraka. India.

\section{0.* Ternstrœmiaceæ.}

24. Thea, L.

†30. sinensis, L. Te, S. and T. Ter. China, Assam.

\section{Malvaceæ.}

25. Althea, I.

†31. rosea, Cav. Hollyhock. N. temp. Eur., Asia.

26. Malvastrum, A. Gray.

32. tricuspidatum, A. Gray. Trop. America.

27. Anoda, Cav.

33. hastata, Cav. Trop. America.

28. Wissadula, Medik.

34. rostrata, Planch. (Leschenaultiana, Mast.)

Cosmop. trop.

29. Hibiscus, L.

35. cannabinus, L. Old World tropical

36. Sabdariffa, L. Rata-bilincha, S. Pulinchakira, T. Rozelle. Old World tropical.

$\checkmark 37$. esculentus, L. Vandakkay, T. Okra. Bandakai. Cosmop. tropical.

i38. Rosa-sinensis, L. Shoeflower. Many forms. Old World tropical.

30. Gossypium, L.

39. herbaceum, I. Cotton. India.

$\uparrow 40$. barbadense, L. Sea island cotton. Trop. America.

31. Adansonia, $l$.

41. digitata, L. Papparappuli, Perukka, T. Baobab, Judas bag. Trop. Africa. 
32. Durio, L.

$\uparrow 42$. zibethinus, L. Durian. Malaya.

\section{4.* Sterculiaceæ.}

33. Cola, Schott.

†43. acuminata, Schott. and Endl. Kola. Trop. Africa.

34. Kleinhovia, L.

44. Hospita, L. Trop. Africa, Malaya.

35. Theobroma, L.

†45. Cacao, L. Chocolat-gas, S. Coco, T. Cacao, Cocoa, Chocolate. Trop. America.

36. Guazuma, Plum.

$\uparrow 46$. tomentosa, Kunth. Trop. America.

36.* Linaceæ.

37. Reinwardtia, Dmrt.

47. trigyna, Planch. India.

38. Erythroxylon, L.

$\uparrow 48$. Coca, L. Coca. Trop. America.

\section{8.* Malpighiaceæ.}

39. Galphimia, Cav.

†49. glauca, Cav. Trop. America.

40.* Geraniaceæ.

40. Tropæolum, L.

50. majus, L. Nasturtium. S. America.

41. Oxalis, $\mathrm{L}$.

51. violacea L. Manikwatte weed. N. America. I.197

42. Averrhoa, L.

†52. Carambola, L. Kamaranga, S.

I.200

†53. Bilimbi, L. Bilin, S. Blimbing. Trop.

America.

I.200

41.* Rutaceæ.

43. Zanthoxylum, L.

†54. Rhetsa, DC. Katukina, S. India.

44. Triphasia, Lour.

55. trifoliata, DC. India, China. 
45. Citrus, L.

†56. Hystrix, DC. Kudalu-dehi, Lima-dehi, S. Caffe lime. Trop. Asia.

†5\%. Aurantium, L. Peni-dodan, S. Narankai, T. Orange. Trop. Asia.

†58. decumana, Murr. Jambola, S. Shaddock, Pomelo. Trop. Asia.

†59. medica, L. var. acida. Dehi, S. Lime. Trop. Asia.

46. Egle, Corr.

60. Marmelos, Corr. Beli, S. Vilvam, T. Bael fruit. India.

\section{2.* Simarubaceæ.}

47. Brucea, Mill.

61. sumatrana, Roxb. Macassar kernels. Trop. Asia, Australia.

\section{Burseraceæ.}

48. Boswellia, Roxb.

62. serrata, Roxb. (glabra, Roxb.). India.

49. Canarium, $\mathrm{L}$.

†3. commune, L. Rata-kekuna, S. Java almond. Moluccas.

\section{Meliaceæ.}

50. Melia, L.

†64. Azedarach, L. Indian lilac, Persian lilac, Bead tree. Himalaya.

1.244

51. Aglaia, Lour.

$\uparrow 65$. odorata, Lour. China.

I.247

52. Swietenia, L.

†66. Mahagoni, L. Mahogany. Trop. America.

$\uparrow 67$. macrophylla. Large-leafed mahogany.

Trop. America.

53. Cedrela, L.

† 68. Toona, Roxb. Red cedar, Indian mahogany. India.

$\dagger 69$. serrata, Royle. Red toon. Himalaya.

\section{4.* Ampelidaceæ.}

54. Vitis, L.

†\%. vinifera, I. Grape. Medit., N. W. India. 
55.* Sapindaceæ.

55. Dittelasma, Hk. f.

71. Raıak, Hk. f. Penela, S. Java, Malacca. 1.300

56. Litchi, Sonnerat.

- †72. chinensis, Sonnerat. Litchi, Leechee. China.

57. Nephelium, L.

†73. lappaceum, L. Rambutan. Malaya.

61.* Anacardiaceæ.

58. Mangifera, L.

†74. indica, L. Amba, S. Mango. India. $\quad 1.318$

59. Anacardium, Rottb.

75. occidentale, L. Caju, S. Cashew. Trop America.

60. Spondias, L.

†r6. dulcis, Forst. f. Cosmop. trop.

63.* Moringaceæ.

61. Moringa, Juss.

7\%. pterygosperma, Gxitn. Murunga, S. Horse-radish tree. N. India.

1.327

65.* Leguminosæ.

62. Crotalaria, I.

78. Willdenowiana, DC. S. Africa. II.18

79. incana, L. Trop. America. II.18

63. Ulex, L.

80. europæus, L. Gorse, Furze, Whin. Europe. II.7

64. Melilotus, Juss.

81. parviflora, Desf. N. temp. zone.

II.21

65. 'Trifolium, L.

8\%. repens, L. White clover, Dutch clover. N. temp. zone.

S3. minus, Sm. N. temp. zone.

St. arvense, L. Hare's foot trefoil. N. temp. zone.

66. Indigofera, L.

8.. Anil, L. Indigo. Anerica.

6\%. Gliricidia, H. B. K.

†86. maculata, H. B. K. Guatemala. 
68. Sesbania, Pers.

87. grandiflora, Pers. Katuru-nurunga, S. Akatti, T. Trop. Asia.

69. Arachis, L.

†88. hypogæa, L. Rata-kaju, S. Groundnut, Peanut, Monkey-nut. Brazil.

71). Desmodium, Desv.

89. diffusum, DC. India.

II.52

71. Uraria, Desv.

†90. crinita, Desv. Trop. Asia.

II.42

72. Lourea, Neck.

91. Vespertilionis, Desv. Tropics generally.

73. Cicer, L.

†92. Arietinum, L. Kadala, S. Chicken pea. Medit.

74. Pisum, L.

93. sativum, L. Pea. Medit.

75. Centrosema, DC.

94. Plumieri, Benth. Trop. America.

76. Periandra, Mart.

95. Berteriana. W. Indies.

77. Erythrina, L.

†96. lithosperma, Bl. Dadap. Java.

†9\%. umbrosa, H. B. K. Madre de cacao. Trop. America.

II. 64

†98. velutina, Willd. W. Indies.

78. Phaseolus, L.

†99. lunatus, L. Bonchi, Damala, S. Curry bean, Lima bean. Cosmop. trop. cult.

$\dagger 100$. vulgaris, L. Potu-bonchi, S. French bean, Kidney bean. Cosmop. trop. cult.

79. Psophocarpus, Neck.

†101. tetragonolobus. Daradamala, S. Burma.

80. Vigna, Savi.

†102. Catiang, Endl. Nil-me, S. Kodippayuru,

T. Cosmop. trop. cult. var. sinensis, Endl. Me-karal, Wandurume, S. Cherry bean.

81. Dolichos, I.

$\uparrow$ 103. biflorus, L. Kollu, Madras gram, Horse gram. India. 
82. Cajanus, DC.

†104. indicus, Spreng. Rata-tora, S. Thavarai,

T. Dhal, Pigeon pea. E. Indies.

II. 80

83. Dalbergia, L. f.

105. latifolia, Roxb. Blackwood, East Indian rosewood. India.

II.88

84. Pterocarpus, L.

106. indicus, Willd. (?) Burma.

85. Cæsalpinia, L.

10\%. Sappan, L. Patangi, S. Sappan. India, Malaya.

II.99

$\dagger$ 108. pulcherrima, Sw. Peacock flower. Cosmop. trop. cul .

$\dagger 109$. coriaria, Thunb. Vanni, T. Divi-divi. Trop. America.

II.101

86. Poinciana, $\mathrm{L}$.

$\dagger 110$. regia, Boj. Flamboyante, Gold mohur. Madagascar.

87. Parkinsonia, L.

111. aculeata, L. Trop. America.

II.102

88. Cassia, L.

$\dagger 112$. nodosa, Ham. Bengal, Malaya.

$\uparrow 113$. grandis, L. f. Horse cassia. Trop.

America.

117. tomentosa, L. Trop. America.

115. hirsuta, L. Trop. America.

III.106

116. lævigata, Willd. Trop. America.

II.106

11\%. alata, L. Rata-tora, S. Tropics gencrally. II.108

†118. glauca, Lam. India, \&c.

†119. multijuga, Rich. Trop. America.

II.109

89. Bauhinia, L.

$\dagger 120$. acuminata, L. India, \&c.

II.116

$\dagger 121$. purpurea, L. India, \&c.

II.117

90. Amherstia, Wall.

†122. nobilis, Wall. Burma.

91. Tamarindus, $\mathrm{L}$.

†123. indica, L. Siyambala, S. Puli, T.

Tamarind. Trop. Africa.

II.114

92. Cynometra, L.

$\dagger 124$. cauliflora, L. Nam-nam. India, Malaya.

93. Parkia, R. Br.

$\dagger 125$. Roxburghii, G. Don. Assam to Malaya. 
94. Neptunia, Lour.

125. plcna, Benth. Trop. America.

95. Desmanthus, Willd. Trop. America.

127. virgatus, Willd. Trop. America.

II. 122

96. Mimosa, L.

128. pudica, L. Nidi-kumba, S. Sensitive plant. Brazil.

II.122

97. Leucæna, Benth.

129. glauca, Benth. Trop. America.

II. 122

98. Acacia, Willd.

†130. decurrens, Willd. Black wattle. Australia.

†131. dealbata, Link. Silver wattle. Australia.

†132. melanoxylon, R. Br. Australian black-

wood, Australia.

†133. longifolia, Willd. Australia.

99. Albizzia, Durazz.

†134. moluccana, Miq. Malay Is.

II.131

100. Pithecolobium, Mart.

$\dagger$ 135. dulce, Benth. Madras thorn. Trop.

America.

II.131

$\dagger 136$. Saman, Benth. Guango, Rain tree. Trop.

America.

II.132

66.* Rosaceæ.

101. Prunus, L.

†13\%. persica, Stokes. Peach. Europe.

102. Spiræa, L.

138. salicifolia, L. Northern tropics.

103. Fragaria, L.

139. vesca, L. Strawberry. N. temp. zone. II.138

104. Rosa, L.

$\uparrow 140$. centifolia, L. Cabbage rose. Caucasus.

$\uparrow 141$. indica, L. Indian or tea rose. India, China.

105. Pyrus, L.

†142. communis, L. Pear. Europe, N. Asia.

67.* Saxifragaceæ.

106. Bauera, Banks.

143. rubioides, Andr. Australia. 


\section{8.* Crassulaceæ.}

107. Bryophyllum, Salisb.

144. calycinum, Salisb. Akkapana, Rata-gowa,

S. Trop. Africa.

II.145

74.* Combretaceæ.

108. Terminalia, L.

†145. Catappa, L. Kottamba, S. Country almond. Malaya.

II.159

109. Quisqualis, L.

$\uparrow 146$. indica, L. India, Malaya.

75.* Myrtaceæ.

110. Eucalyptus, L'Her.

$\dagger 14 \%$. Globulus, Labill. Blue gum. Australia.

†148. diversicolor, F. Muell. Australia.

†149 Leucoxylon, F. Muell. Ironbark. Australia.

II. 166

II. 166

11.166

†150. robusta, Sm. Swamp mahogany. Australia.

†151. marginata, Sm. Jarrah. Australia.

II.166

11.166

II. 166

111. Psidium, L.

152 Guajava, L. Pera, S. Guava. Trop. America.

11.167

112. Eugenia, L.

†153. malaccensis, L. Malay apple. Malaya. 11.170

†154. Jambos, L. Jambu, S. Rose apple. Malaya.

II. 170

†155. Michelii, Lam. Rata-jambu, S. Brazil cherry. Trop. America.

†156. caryophyllata, Thunb. Clove. Moluc-

II. 188 cas.

\section{6.* Melastomaceæ.}

113. Tibouchina, Aubl.

$\uparrow$ 15\%. semidecandra, Cogn. Brazil. (Pleroma macranthum, Hk. f.)

77.* Lythraceæ.

114. Punica, L.

†158. granatum, L. Delun, S. Madalankai, T.

Pomegranate. N. W. India, Persia, \&c. 
78.* Onagraceæ.

115. Enothera, L.

159. fruticosa, L. N. America.

II.235

160. odorata, Jacq. (?) Chili.

161. speciosa, Nutt. N. America.

\section{1.* Turneraceæ.}

116. Turnera, L.

162. ulmifolia, L. Trop. America.

II. 239

\section{2.* Passifloraceæ.}

117. Passiflora, L.

163. suberosa, L. W. Indies.

164. quadrangularis, L. Granadilla. Trop.

II.241

America.

165. fœtida, L. Trop. America.

166. edulis, Sims. Sweet cup, Passion fruit. Brazil.

167. stipulata, Aubl. Trop. America.

168. laurifolia, L. Water lemon. Trop. America.

118. Carica, L.

†169. Papaya, L. Pepol, S. Pappali, T. Papaw. Trop. America.

$†$ 170. candamarcensis, Hk. f. Mountain papaw. Ecuador.

\section{3.* Cucurbitaceæ.}

119. Trichosanthes, $\mathrm{L}$.

$\uparrow 171$. Anguina, L. Patola, S. Podivilanga, T. Snake gourd. Trop. Asia.

II. 245

120. Lagenaria, Ser.

†172. vulgaris, Ser. Diya-labu, S. Churai, T.

Bottle gourd, Calabash cucumber. Tropics.

II. 247

121. Cucumis, $\mathrm{L}$.

†173. sativus, L. Rata-kekiri, S. Cucumber.

India.

122. Citrullus, Neck.

†174. vulgaris, Schrad. Komadu, S. Water melon. Trop. Africa.

II. 253

123. Benincasa, Savi.

†175. cerifera, Savi. Alu-puhul, S. Puchini,

T. Ash pumpkin. E. tropical cult. 
124. Cucurbita, L.

†16. maxima, Duch. Gourd, Pumpkin.

Tropics generally.

$\lceil 17 \%$. moschata, Duch. Rata-labu, S. Origin unknown.

†178. Pepo, L. Pumpkin, Vegetable marrow.

N. America.

125. Sechium, P. Browne.

$\dagger 179$. edule, Sw. Chocho, Chayote. Trop.

America.

86.* Cactace

126. Opuntia, Mill.

180. Dillenii, Haw. Mexico.

88.* Umbelliferæ.

127. Apium, $\mathrm{L}$.

†181. graveolens, L. Celery. Europe, N. W. Asia.

128. Carum, L.

182. Roxburghianum, Benth. \& $\mathrm{Hk}$ f. Indo-Malaya.

II. 278

183. Petroselinum, B. \& Hk. f. Pursley. Medit.

129. Peucedanum, L.

†184. sativum, B. \& Hk. f. Parsnip. N. temp. zone.

130. Coriandrum, L.

†185. sativum, L. Coriander. Medit.

131. Daucus, L.

†186. Carota, L. Carrot. N. temp. zone. Old World.

\section{9.* Araliaceæ.}

132. Panax, L.

$\uparrow 187$. fruticosum, L. Malaya.

II. 282

133. Fatsia, Dcne. \& Pl.

188. papyrifera, B. \& Hk f. Rice paper tree. China. 


\section{( 137$)$ \\ GAMOPETALE.}

92.* Rubiaceæ.

134. Cinchona, L.

†189. Calisaya, Wedd. Yellow bark, Crown bark. Trop. S. America.

†190. succirubra, Pav. Red bark. Trop. S. America.

†191. officinalis, L. Crown bark, Brown bark. Trop. S. America.

135. Oldenlandia, L.

192. crystallina, Roxb. India.

II.315

136. Coffea, L.

†193. arabica, L. Kopi, S. and T. Arabian coffee. Trop. Africa.

$\dagger 194$. liberica, Hiern. Liberian coffee. Trop. Africa.

96.* Compositæ.

13\%. Ageratum, L.

195. conyzoides, L. Hulan-tala, S. Pumpulla, T. White weed, Goat weed. Trop. America.

III.353

III.13

138. Mikania, Willd.

196. scandens, Willd. India, trop. America.

139. Erigeron, L.

197. linifolins, Willd. W. temp. Asia.

III. 17

140. Gnaphalium, L.

198. indicum, L. (multicaule, Willd.). Wild mignonette. Tropies generally.

III.32

141. Helichrysum, Gærtn.

199. bracteatum, Willd. Australia.

III.33

142. Carpesium, L.

200. cernuum, L. Temp. Asia.

III. 34

143. Lagascea, Cav.

201. mollis, Cav. Trop. America.

III.34

144. Melampodium, L.

202. paludosum, H. B. K. (divaricatum. DC.).

Ran-manissa. S. America.

145. Tithonia, Desf.

203. diversifolia, A. Gray. Wild sunflower. Mexico, \&c. 
146. Helianthus, $\mathrm{L}$.

204. annuus, L. Sunflower. N. America.

205. tuberosus, L. Jerusalem artichoke. N.

America.

147. Synedrella, Gærtn.

206. nodiflora, Gærtn. Mexico.

148. Cosmos, Cav.

20\%. bipinnatus, Cav. Mexico.

III.40

208. sulphureus, Cav. Mexico.

III.40

149. Galinsoga, Ruiz. \& Pav.

209. parviflora, Cav. Peru

III.42

150. Tridax, L.

210. procumbens, L. S. America.

III.42

151. Tagetes, L.

211. erecta, L. Mexico.

212. patula, L. Mexico.

III.42

III. 42

152. Cotula, L.

213. australis, Hk. f. Australia.

III.42

153. Artemisia, L.

214. Roxburghiana, Bess. Himalaya.

154. Cynara, L.

†215. Cardunculus, L. Artichoke. Medit.

155. Taraxacum, L.

216. officinale, Wigg. Dandelion. Temp. zone.

III.51

156. Lactuca, I.

†21\%. Scariola, L. Lettuce. Europe, N. W. Asia.

15\%. Sonchus, L.

218. asper, Vill. Sow thistle. N. temp. zone. III.52

219. oleraceus, L. Sow thistle. N. temp. zone. III.52

99.* Lobeliaceæ.

158. Isotoma, Lindl.

220. longiflora, Presl. W. Indies.

III.58

107.* Plumbaginaceæ.

159. Plumbago, $\mathrm{L}$.

†221. rosea, L. Rat-netul, S. India.

III. 65 
108.* Primulaceæ.

160. Anagallis, L.

222. arvensis, L. var. cœrulea, Lam. Pimpernel, Poor man's weather glass. N. temp. zone, Old World.

III. 66

109.* Myrsinaceæ.

161. Ardisia, Sw.

†223. solanacea, Roxb. Balu-dan, S. Trop. Asia cult.

III.74

110. Sapotaceæ.

162. Achras, L.

†224. Sapota, L. Sapodilla plum. Trop. America.

\section{3.* Oleaceæ.}

163. Jasminum, L.

$\nmid 225$. Sambac, Ait. Pichcha, Geta-pichcha, S. Arabian jasmine. Trop. Asia.

†226. pubescens, Willd. Trop. Asia.

22\%. laurifolium, Roxb. N. E. India.

III.113

III. 113

III.114

164. Nyctanthes, $\mathrm{L}$.

†228. arbor-tristis, L. Sepala, Sepalika, S. India.

III.116

115.* Apocynaceæ.

165. Allamanda, L.

229. Cathartica, L. Wal-ruk-attana, S.

Brazil.

III.124

166. Landolphia, Beauv.

†230. Kirkii, Dyer. African rubber. Trop. Africa.

167. Vinca, L.

231. rosea, L. Madagascar periwinkle. Cosmop. trop.

232. major, L. Periwinkle. Medit.

III.130

168. Plumeria, $\mathrm{L}$.

†233. acutifolia, Poir. Alariya, S. Temple tree.

III.I30

169. Alstonia, R. Br.

234. macrophylla, IVall. Malaya.

t Cultivated only. 
170. Tabernæmontana, L.

$\nmid 235$. Coronaria, Br. Origin unknown.

III.133

171. Vallaris, Burm.

$\nmid 236$. Pergulana, Burm. Malaya.

III. 155

172. Nerium, L.

23\%. Oleander, L. Oleander. Medit. to Japan.

\section{6.* Asclepiadaceæ.}

173. Cryptostegia, R. Br.

238. grandiflora, Br. Trop. Africa.

III.145

174. Gomphocarpus, R. Br.

239. fruticosus, R. Br. Africa.

175. Asclepias, L.

240. curassavica, L. Wild ipecacuanha. W. Indies.

III.149

122.* Convolvulace

176. Argyreia, Lour.

241. speciosa, Sweet. Maha-dumudu, S. Bengal.

III.207

17\%. Ipomæa, L.

242. cissoides, Griseb. Trop. America.

III.212

243. Batatas, Lam. Batala, S. Sweet polato.

Trop. America.

III.212

$\uparrow 244$. muricata, Jacq. India.

III.214

245. tuberosa, L. W. Indies.

III. 224

246. sidæfolia, Choisy. Trop. America.

III. 220

24\%. coccinea, L. Trop. America.

III.215

248. Quamoclit, L. Rala-pamba, S. Trop. America.

III.215

178. Porana, Burm.

†249. paniculata, Roxb. India, Java.

III. 227

123. Solanaceæ.

179. Lycopersicum, Mill.

†250. esculentum, Mill. Rata-batu, S. Takkali,

S. and T. Tomato, Love apple.

180. Solanum, L.

†251. tuberosum, L. Potato. S. America.

252. ciliatum, Lam. Brazil.

III.234

†253. macranthum, Dun. Potato tree. Brazil.

†254. melongena, L. Wambatu, S. Brinjal, Egg plant. 
181. Cyphomandra, Sendtn.

†255. betacea, Sendtn. Tree tomato. S. America.

182. Physalis, $\mathrm{L}$.

256. angulata, L. Tropics generally.

25\%. peruviana, L. Cape gooseberry, Straw.

III.237

berry, or Gooseberry tomato. Trop. America. III.237

183. Capsicum, L.

258. minimum, Roxb. Nayi-miris. Bird pepper. Tropics generally.

†259. annuum, L. Chilly, Red pepper. Tropies generally.

184. Nicandra, Adans.

260. physaloides, Gærtn. Peru.

III.238

185. Datura, L.

261. Stramonium, L. Thorn apple. Cosmopolitan.

†262. suaveolens, H. \& B. Rata-attana, S.

Trumpet flower. Mexico.

III.238

186. Cestrum, L.

III.239

III. 239

$\nmid 263$. fasciculatum, Miers. Mexico.

187. Nicotiana, L.

†264. Tabacum, L. Tobacco. S. America.

188. Browallia, L.

265. viscosa, H. B. K. S. America.

189. Brunfelsia, L.

266. uniflora, D. Don. Origin unknown.

124.* Scrophulariaceæ.

190. Verbascum, L.

26\%. Thapsus, L. Mullein. Old World, N.

temp. zone.

III.241

191. Calceolaria, L.

268. chelidonioides, H. B. K. Mexico.

III.241

192. Maurandia, Ort.

269. scandens, A. Gray. Mexico.

193. Stemodia, L.

270. parviflora, Ait. Trop. America.

III.242

194. Scoparia, L.

271. dulcis, L. Trop. America.

III. 255

$\dagger$ Cultivatod only. 
195. Veronica, L.

272. didyma, Tenore. (polita, Fries.) Speedwell. N. temp. zone, Old World.

III.255

\section{8.* Gesneraceæ.}

196. Rhynchoglossum, $\mathrm{Bl}$.

273. zeylanicum, Hook. India.

III.279

\section{9.* Bignoniaceæ.}

197. Millingtonia, L. f.

†274. hortensis, L. f. Indian cork tree. Burma. III.282

198. Spathodea, P. Br.

†275. campanulata, Beauv. Trop. Africa.

III.282

199. Stereospermum, Cham.

†276. suaveolens, DC. Palol, Ela-palol, S. India.

III.284

\section{0.* Pedaliaceæ.}

200. Martynia, L.

27\%. diandra, Glox. Naka-tali, T. Tigers' claws. Mexico.

III.285

201. Sesamum, L.

278. occidentale, Heer \& Regel. Origin unknown.

III.286

131.* Acanthaceæ.

202. Thunbergia, L. f.

279. alata, Boj. Trop. Africa.

280. laurifolia, Lindl. Malaya.

III.289

203. Barleria, L.

†281. cristata, L. India, Burma.

III.321

134.* Verbenaceæ.

204. Lantana, L.

282. trifolia, L. Trop. America.

283. aculeata, L. Rata-hinguru, Gandapana, S.

Lantana.

III.346

III.346

205. Stachytarpheta, Vahl.

†284. mutabilis, Vahl. Trop. America.

206. Verbena, L.

285. venosa, Gill \& Hook. Brazil.

III.349 
207. Duranta, L.

†286. Plumieri, Jacq. Trop. America.

208. Tectona, L. f.

$\dagger 28 \%$ grandis, L. f. Teak. India, Burma.

209. Clerodendron, $\mathrm{L}$.

288. Siphonanthus, Br. India.

III.361

135.* Labiatæ.

210. Ocimum, L.

†289. basilicum, L. Suvandu-tala, S. Sweet basil. Trop. Asia.

III.366

211. Plectranthus, L'Her.

$\uparrow 290$. zeylanicus, Benth. Iri-weriya, S. Trop. Asia.

III.371

212. Coleus, Lour.

$\dagger 291$. parviflorus, Benth. (Plectranthus tuberosus, Bl.) Innala, S. Country potato.

India.

292. aromaticus, Benth. Kapuru-walliya, S. India.

III.374

III. 374

213. Mentha, L.

293. sylvestris, L. var. crispa, Benth. Mint.

Europe.

III 381

214. Salvia, L.

294. coccinea, L. Trop. America.

136.* Plantaginaceæ.

215. Plantago, L.

295. lanceolata, L. Plantain. N. temp. Eur., Asia.

III.389

\section{INCOMPLET AE.}

137.* Nyctaginaceæ.

216. Mirabilis, $\mathbf{L}$.

296. Jalapa, L. Sendrikka, S. Marvel of Peru, False jalap. Peru.

III.391

217. Bougainvillæa, Comm.

297. spectabilis, Willd. Brazil. 
218. Pisonia, L.

298. morindæfolia, Br. Lechchaikedda, Chandi,

T. Lettuce tree. (Wata-banga-kola.) Malaya,

Polynesia, \&c.

III.392

139.* Amarantaceæ.

219. Amarantus, L.

299. caudatus, L. Love-lies-bleeding. Medit.

to India.

III.396

300. hypochondriacus, L. Prince of Wales's

feather. N. America.

301. paniculatus, L. (frumentaceus, Ham.)

Ranatampala, S. N. America.

†302. oleraceus. Tampala, S. Egypt, India.

III.396

III.396

220. Gomphrena, L.

†303. globosa, L. Globe amaranth. Tropical America.

140.* Chenopodiaceæ.

221. Chenopodium, L.

304. murale, L. Temp. zone.

305. ambrosioides, L. Wormseed. Temp. and trop.

III. 407

306. opulifolium, Schrad. N. temp. zone.

III. 407

III.407

222. Beta, L.

†30\%. vulgaris, L. Beetroot. Europe.

141.* Phytolaccaceæ.

223. Rivina, L.

308. humilis, L. Trop. America.

III. 140

224. Mohlana, Mart.

309. nemoralis, Mart. Trop. America and Africa.

III.410

225. Phytolacca, L.

310. octandra, Moq. Trop. America.

III. 410

143.* Polygonaceæ.

226. Polygonum, L.

311. molle, D. Don. Himalaya. 
227. Rumex, L.

312. obtusifolius, L. N. temp. zone.

313. crispus, L. Europe, N. Asia.

314. Acetosella, L. Europe, N. Asia.

228. Antigonon, Andl.

†315. leptopus, H. \& A. S. America.

\section{8.* Piperaceæ.}

229. Peperomia, Ruiz. \& Pav.

316. Fraseri, Cas. DC. Ecuador.

\section{0.* Myristicaceæ.}

230. Myristica, L.

†31\%. fragrans, Houtt. Nutmeg and Mace.

Moluccas.

\section{2.* Lauraceæ.}

231. Cinnamomum, B1.

†318. Camphora, Nees and Eberm. Camphor. China, Japan, Formosa.

232. Persea, Gærtn.

319. gratissima, Gærtn. Avocado, Alligator pear,

Palta. Trop. America.

\section{3.* Proteaceæ.}

233. Grevillea, R. Br.

†320. robusta, A. Cunn. Silky oak. Australia. III.457

160.* Euphorbiaceæ.

234. Euphorbia, L.

†321. pulcherrima, Willd. Poinsettia. Mexico.

†322. neriifolia, L. Patak, S. India.

IV.5

†323. Tirucalli, L. Nawahandi, S. Kalli, T. Milk hedge. Trop. Africa.

IV.5

235. Phyllanthus, L.

†324. longifolius, Jacq. Rata-nelli, Siri-nelli, S. Malaya.

IV.26

236. Hevea, Aubl.

†325. brasiliensis, Muell. Arg. Para rubber. Trop.

S. America. 
237. Jatropha, L.

326. gossypifolia, L. Trop. America.

+32\%. Curcas, L. Rata-endaru, S. Kadda-

manakku, T. Physic nut. Tropics generally. IV.46

238. Aleurites, Forst.

$\dagger 328$. triloba, Forst. Rata-kekuna, Tel-kekuna. Candle nut. Polynesia.

IV.46

239. Croton, L.

†329. Tiglium, L. Jayapala, S. Nervalam, T.

Croton oil plant. India, Malaya.

IV.49

240. Codiæum, Rumph.

†330. variegatum, Bl. Croton. Polynesia.

IV.52

241. Manihot, Adans.

†331. utilissima, Pohl. Manyokka, S. Manioca, Cassava, Tapioca. Brazil.

†332. Glaziovii, Muell. Arg. Ceara rubber. Brazil.

333. dichotoma, Ule. Jequié rubber. Brazil.

334. piauhyensis, Ule. Remanso rubber. Brazil.

242. Acalypha, L.

†33.5. Many garden varieties.

243. Ricinus, L.

336. Communis, L. Endaru, S. Chittamanak$k u, T$. Castor oil. Africa.

IV.72

244. Sapium, P. Br.

33\%. sebiferum, Roxb. Tallow tree. China. IV.76

\section{2.* Urticaceæ.}

245. Cannabis, $\mathrm{L}$.

†338. sativa, L. Hemp (ganja). Central Asia.

246. Broussonetia, L'Herit.

339. papyrifera, Vent. Paper mulberry. Malaya, Polynesia.

247. Morus, L.

†340. alba, L. var. indica, L. Indian mulberry. India.

248. Ficus, L.

341. religiosa, L. Bo, S. Arachu, T. Himalaya.

†342. elastica, Roxb. Assam india rubber, Rambong. Trop. Asia. 


\section{$(147)$}

24.9. Castilloa, Cervant.

$\dagger 343$. elastica, Cervant. Mexico.

250. Artocarpus, L.

†344. integrifolia, L. f. Kos, S. Pila, T. Jak.

India.

IV.99

†345. incisa, L. Rata-del, S. Erapilakai, T.

Breadfruit. Malaya, Polynesia.

2.51. Pilea, Lindl.

346. muscosa, Lindl. Gunpowder plant. S. America.

IV.108

167.* Casuarinaceæ.

252. Casuarina, Forst.

34\%. equisetifolia, Forst. Kasa, S. Chavukku,

T. Burma, Malaya.

IV.120

\section{MONOCOT YLEDONS.}

\section{5.* Orchidaceæ.}

253. Vanilla, Sw.

$\nmid 348$. planifolia, Andr. Vanilla. Mexico.

\section{Zingiberaceæ. $\ddagger$}

254. Kæmpferia, L.

†319. Galanga, L. Hinguru-piyali. Trop. Asia. IV.244

255. Curcuma, L.

†350. longa, L. Kaha, S. Manchal, T.

Turmeric. Trop. Asia.

IV.242

256. Zingiber, Adans.

$\nmid 351$. officinale, Rosc. Inguru, S. Inji, T.

Ginger. Tropical.

25\%. Alpinia, L.

352. Galanga, Sw. Galangal, Kalu-wala, S.

Trop. Asia.

IV. 249

353. calcarata, Rosc. Katakiriya, S. India, China.

IV.249

258. Maranta, L.

†5 . arundinacea, L. Arrouroot. S. America.

$\dagger$ Cultivated only.

f This and Musaceæ are combined into Scitamineæ in the Flora proper. 


\section{8.* Bromeliaceæ.}

259. Ananas, Tourn.

355. sativus, Schult. Annasi, S. Pineapple. Trop. America.

\section{0** Iridaceæ.}

260. Crocosmia, Planch.

356. aurea, Planch. (Tritoria aurea, Pappe.)

Trop. and S. Africa.

\section{1.* Amaryllidaceæ.}

261. Curculigo, Gærtn.

$\nmid 35 \%$. recurvata, Dryand. Waga-pol, S. Trop. Asia.

262. Agave, L.

†358. americana, L. Century plant, American aloe. Trop. America.

263. Furcrea, Vent.

359. gigantea, Vent. Mauritius hemp. Trop. America.

\section{3.* Dioscoreaceæ.}

264. Dioscorea, L.

360. alata, L. Kiri-kondol, S. Trop. Asia. IV.279 361. spinosa, Roxb. India.

362. purpurea, Roxb. Kahata-kondol, S. India.

363. sativa, L. Katu-kukul-ala, S. Tropics.

\section{5.* Liliaceæ.}

265. Phormium, Forst.

364. tenax, Forst. New Zealand flax. New Zealand.

266. Aloe, L.

365. vera, L. var. littoralis, Kœn. N. Africa. IV.211 
267. Cordyline, Comm.

†366. terminalis, Kunth. Dracana. Asia, Australia.

268. Allium, L.

†36\%. Cepa, L. Onion. Europe.

†368. Porrum, L. Leek. Europe.

†369. sativum, L. Garlic. Europe.

\section{Palmæ.}

269. Oreodoxa, Willd.

†3\% . regia, Kunth. Royal palm. Cuba,

Panama.

270. Hyphæne, Gærtn.

†371. thebaica, Mart. Dum palm. Trop. Africa.

271. Elæis, Jacq.

†37. guineensis, Jacq. Oil palm. Trop. Africa.

195.* Pandanaceæ.

272. Pandanus, L. f.

3\%3. Kaida, Kurz. India.

198.* Araceæ.

273. Richardia, Kunth.

374. africana, Kunth. Arum lily. S. Africa.

274. Alocasia, Schott.

375. indica, Schott. Rata-ala, Desa-ala, S.

India, Malaya.

202.* Naiadaceæ.

275. Aponogeton, Thunb.

376. distachyum, Thunb. Cape pond-weed.

S. Africa.

207. Gramineæ.

276. Zea, L.

†37\%. Mays, L. Iringu, S. Maize, Indian corn. Trop. America.

277. Saccharum, L.

$\nmid 378$. officinarum, L. Sugar cane. Tropical. 


\section{$(150)$}

278. Sorghum.

379. vulgare, Pers. Karal-iringu, T. Cholam, Guinea corn, Millet. Tropical and subtropical.

279. Cymbopogon, Hack.

†380. citratus, Stapf. Sera, S. Lemon grass. India.

280. Setaria, P. Br.

381. italica, Beauv. Tanahal, S.'Italian millet. Tropical and subtropical.

281. Anthoxanthum, L.

382. odoratum, L. Sweet vernal grass. N. temp. zone, Old World.

V.305

282. Eleusine, Gærtn.

†383. Coracana, Gærtn. Kurakkan, Rayi. India.

283. Dactylis, L.

384. glomerata, L. Cock's foot. Europe, N. Asia.

284. Dendrocalamus, Nees.

$\dagger 385$. giganteus, Munro. Giant bamboo. Burma.

GYMNOSPERMAE.

209.* Coniferæ.

285. Cupressus, L.

†386. Lindleyi, Klotzsch. (Knightiana, Perry.)

Mexico.

†387. macrocarpa, Hartn. Monterey cypress.

California. 


\section{INDEX}

By M. TILLIS.

The numbers in the Latin list refer to the genera: those in italics to genera in the Supplement.

The number 8 in the other lists refer to species.

\section{LATIN NAMES.}

Synonyms in italics, with equivalents beside them.

Abelmoschus, Hibiscus

Aberia, 54

Abrus, 231

Abutilon, 90

Acacia, 272, 98

Acalypha, 724, 242

Acampe, Saccolabium

Acanthaceæ, p. 65, p. 142

Acanthephippium, 788

Acanthus, 596

Achras, 162

Achyranthes, 653

Acor'us, 911

Acranthera, 375

Acronychia, 127

Acrostichum, 1085

Acrotrema, 8

Actephila, 701

Actiniopteris, 1071

Actinodapline, 679

Actinoschœnus, 935

Adansonia, 31

Adenanthera, 270

Adenochlæena, 725

Adenosma, 553

Adenostemma, 410

Adlhatoda, 606
Adiantum, 1070

Adina, 361

Adinandra, 75

Adrorhizon, 785

Aicgiceras, 459

Aginetia, 568

Agle, 46

Aluropus, 1020

Asrides, 805

Erua, 652

Eschynanthus, 572

Eschynomene, 223

Aganosma, 490

Agave, 262

Ageratum, 137

Aglaia, 149, 51

Agrimonia, 280

Agrostistachys, 722

Agrostophyllum, 793

Agyneia, 702

Ailantus, 137

Alangium, 356

Albizzia, 99

Alchemilla, 278

Alaurites, 238

Alisma, 915

Alismaceæ, p. 99 
Allæanthus, 752

Allamanda, 165

Allantodia, Diplaziopsis

Allium, 868, 268

Allmania, 645

Alloophania, 368

Allophylus, 191

Alocasia, 907, 27t

Aloe, 266

Alphonsea, 26

Alpinia, 844, 257

Alsendaphne, 678

Alsodeia, 50

Alsophila, 1040

Alstonia, 485, 169

Alternanthera, 654

Althea, 25

Alvisia, 790

Alyssicarpns, 229

Alyxia, 478

Amanoa, Cleistanthus

Amarantaceæ, p. 72, p. 144

Amarantus, 647, 219

Amaryllidaceæ, p. 93, p. 148

Amherstia, 90

Ammannia, 309

Amomum, 845

Amoora, 152

Amorphophallus, 903

Ampelidex, p. 20, p. 12.9

Amphicosmia, Hemitelia

Anacardiace $æ$, p. 22, p. 1.30

Anacardium, 59

Anagallis, 160

Anamirta, 28

Ananas, 259

Anaphalis, 423

Anaxagorea, 19

Ancistrocladaceæ, p. 11

Ancistrocladus, 88

Andrographis, 601

Andropogon, 984

Aneilema, 878

Anemone, 3

Angiopteris, 1091

Anisochilus, 631

Anisogonium, Diplazium

Anisomeles, 637

Anisophyllea, 293

Anoda, 27

Anodendron, 492

Ancectochilus, 823
Anogeissus, 295

Anogramma, 1064

Anona, 5

Anonaceax, p. 3, p. 12.5

Anotis, 372

Anthistiria, 988

Anthocephalus, 360

Anthoxanthum, 281

Antiaris, 745

Antidesma, 714

Antigonon, 228

Antrophyum, 107 7

Aphyllorchis, 833

Apium, $12 \pi$

Apluda, 983

Apocopis, 981

A pocynaceæ, p. 32, p. 13.9

Apodytes, 165

Aponogeton, 917, 27.;

Aporosa, 712

Apostasia, 838

Aracex, p. 98, p. 149

Arachis, 69

Araliaceæ, p. 40, p. 136

Ardisia, 458, 161

Areca, 884

Argemone, 6

Argyreia, 539, 176

Arisema, 900

Iristida, 990

Aristolochia, 668

Aristolochiacer. p. 74

Artabotrys, 16

Artanema, 557

Artemisia, 436, 15.3

Arthraxon, 982

Arthrocnemum, 656

Arthropteris, 1048

Artocarpus, 747, 2.50

Arundina, 792

Arundinaria, 1024

Arundinella, 959

Asclepiadacex, p. 55, p. 140

Asclepias, 175

Asparagus, 863

Aspidium, 1043

Asplenium, 1060

Asystasia, 599

Atelantia, 135

Athyrium, 1057

Atriplex, 655

Atylosia, 247 
Arena, 996

Avelrhoa, $1: 2$

Avicennia, 624

Axinandra, 315

Axonopus, 953

Azadirachta, 147

Azima, 474

Azolla, 1034

Baissea, 491

Balanocarpus, 84

Balanophora, 696

Balanophoraceæ, p. is

Balsamodendron, 142

Bambusa, 1025

Barleria, 597, 203

Barringtonia, 301

Basella, 659

Bassia, 463

Bauera, 106

Bauhinia, 267, 89

Begonia, 340

Begoniacex, p. 61

Beilschniedia, 675

Benincasa, 12:3

Berberidaceæ, p. 5

Berberis, 77

Bergia, 68

Berrya, 109

Beta, 222

Bidens, 433

Bignoniaceæ, p. 64, p. 142

Biophytum, 121

Bixa, 16

Bixaceæ, p. 6, p. 126

Blachia, 720

Blainvillea, 430

Blechnum, 1061

Blepharis, 595

Blepharispermum, 422

Blumea, 418

Blyxa, 771

'Bocagea, 723

Bocconia, 7

Boehmeria, 762

Bnerhaavia, 642

Bombax, 98

Bonnay a, 561

Boraginacer, p. 59 :

Borassus, 892

Boswellia, 18

Botrychium, 1094
Boucher, 614

Bougainvillæa, 217

Brachypodium, 1022

Bragantia, 667

Brassica, 10

Breweria, 545

Breynia, 707

Bridelia, 699

Bromeliacere, p. 148

Broussonetia, 2.10

Browallia, 188

Brucea, 47

Bruguiera, 290

Brunfelsia, 189

Bryonia, 330

Bryophyllum, $10 \%$

Buchanania, 201

Bulbophyllum, 782

Bulbostylis, 929

Bupleurum, 349

Burmannia, 776

Burmanniacex, 1). 86

Burseracece, p. 17, p. 129

Butea, 240

Byrsophyllum, 380

Cactaceæ, p. 39, p. 136

Cadaba, 46

Cæsalpinia, 258

Cajanus, 82

Calamagrostis, 995

Calamintha, 635

Calamus, 891

Calanthe, 796

Calceolaria, 191

Callicarpa, 617

Callitriche, 287

Calophanes, 589

Calophyllum, 71

Calotropis, 499

Camellia, Thea

Campanula, 449

Campanulacэr, p. 49

Campbellia, 570

Campnosperma, 206

Cananga, 3

Canarium, 143, 1!9

Canavalia, 241

Canna, 851

Cannabis, 245

Canscora, 522

Cansjera, 161 
Canthium, 391

Capparidəæ, p. 5, p. 126

Capparis, 47

Caprifoliaceæ, p. 40

Capsella, 11

Capsicum, 183

Carallia, 291

Caralluma, 515

Carapa, 154

Cardamine, 41

Cardanthera, 587

Cardiospermum, 189

Carex, 945

Careya, 302

Carica, 118

Carissa, 476

Carpesium, 142

Carum, 350, 128

Caryophyllaceæ, p. 7, p. 126

Caryota, 887

Casearia, 319

Cassia, 261, 88

Cassytha, 682

Castilloa, 249

Casuarina, 252

Casuarinaceæ, p. 147

Cedrela, 53

Celastraceæ, p. 19

Celastrus, 175

Celosia, 644

Celsia, 552

Celtis, 741

Centipeda, 435

Centotheca, 1019

Centranthera, $\mathbf{5 6 6}$

Centrosema, 75

Cephalandra, 325

Cerasiocarpum, 336

Cerastium, 61

Ceratophyllaceæ, p. 85

Ceratopliyllum, 767

Ceratopteris, 1086

Cerbera, 480

Ceriops, 289

Ceropegia, 514

Cestrum, 186

Chætocarpus, 736

Chailletia, 157

Chailletiaceæ, p. 18

Chamabainia, 763

Chamxraphis, 952

Championia, 575
Chasalia, 398

Chavica, Piper, 669

Cheilanthes, 1069

Cheirostylis, 821

Chenopodiacer, p. 73, p. 144

Chenopodium, 221

Chickrassia, 155

Chirita, 574

Chloranthaceæ, p. 75

Chloranthus, 671

Chloris, 1004

Chlorophytum, 867

Chloroxylon, 156

Chonemorpla, 489

Christisonia, 569

Chrozophora, 723

Chrysoglossum, 787

Chrysogonum, 426

Chrysophyllum, 460

Chrysopogon, Andropogon

Cicer, 73

Cinchona, 134

Cinnamomum, 676, 231

Cipadessa, 148

Cirrhopetalum, 783

Cissampelos, 35

Citrullus, 12\%

Citrus, 45

Cladium, 937

Claoxylon, 729

Clausena, 131

Cleidion, 731

Cleisostoma, 811

Cleistanthus, 700

Clematis, 1

Cleome, 42

Clerodendion, 621, 209

Clinogyne, 849

Clitoria, 245

Cocculus, 32

Cochlearia, 9

Cochlospermum, 15

Cocos, 893

Codiæum, 240

Colachne, 999

Cologyne, 784

Coffea, 394, 136

Coix, 969

Cola, 33

Coldenia, 531

Colous, 630, 212

Colocasia 906 
Colubrina, 185

Combretaceæ, p. 32, p. 134

Combretum, 297

Commelina, 877

Commelinaceæ, p. 115

Compositæ, p. 48, p. 137

Coniferæ, p. 150

Coniogramme, 1065

Connaraceæ, p. 23

Connarus, 209

Convolvulaceæ, p. 59, p. 140

Convolvulus, 543 .

Conyza, 417

Corallocarpus, 335

Corchorus, 112

Cordia, 529

Cordyline, 267

Coriandrum, 130

Cornacєæ, p. 40

Corymbis, 827

Corypha, 890

Coseinium, 29

Cosmos, 148

Cosmostigma, 508

Costus, 843

Cottonia, 813

Cotula, 152

Crassulaceæ, p. 31, p. 134

Cratæva, 45

Crawfurdia, 525

Crepis, 441

Cressa, 546

Crinum, 856

Crocosma, 261

Crossandra, 598

Crotalaria, 213, 62

Croton, 716, 239

Cruciferæ, p. 5, p. 125

Crudia, 264

Cryptocarya, 674

Cryptocoryne, 898

Cryptolepis, 495

Cryptostegia, 173

Cryptostylis, 819

Ctenolepis, 337

Cucumis, 327, 121

Cucurbita, 124

Cucurbitacere, p. 37, p. 13.5

Cudrania, 746

Cullenia, 100

Cuprossus, 28.5

Cureuligo, 855,260

Curcuma, 840, 255

Cuseuta, 547

Cyanotis, 879

Cyathea, 1038

Cyatheacer, p. 114

Cyathocalyx, 15

Cyathula, 648

Cyc adaceæ, p. 86

Cycas, 768

Cyelea, 36

Cyclophorus, 1082

Cyclostemon, 710

Cymbidium, 799

Cymbopogon, 986, 279

Cymodocea, 921

Cynanchum, 503.

Cynara, 154

Cynodon, 1003

Cynoglossum, 536

Cynometra, 262, 92

Cyperaceæ, p. 100)

Cyperus, 924

Cyphomandra, 181

Cyphostigma, 847

Dactylis, 283

Dædalacanthus, 592

Dæmia, 501

Dalbergia, 252,83

Dalochampia, 734

Daphniphyllum, 713

Datiscacere, p. 39

Datura, 551, 185

Daucus, 131

Davallia, $105 \mathrm{I}$

Debregeasia, 766

Delima, 6

Dendrobium, 781

Dendrocalamus, 284

Dennstredtia, 1054

Dentella, 366

Derris, 255

Desmanthus, 95

Desinodium, 230, 70

Diacalpo, 1041

Dialium, 263

Dianella, 865

Direllostyles, 95

Dichretaria, 1007

Dichilanthe, 389

Dichrocephala, 411

Dichrostachys, 271 
Dicliptera, 611

Dicræa, 662

Didymocarpus, 573

Digera, 646

Jilleniacer, p. 2

Dimeria, 971

Dimorplocalyx, 721

Dinebra, 1006

Dioclea, 242

Dioscolea, 859, 264

Dioscoreacexe, p. 94, p. 148

Diospyros, 467

Dipeadi, 869

Diplachne, 1016

Diplacrum, 944

Diplocentrum, 808

Diplanthera, 922

Diplaziopsis 1059

Diplazium, 1058

Diploprora, 815

Diplospora, 385

Dipsacex, p. 25

Dipsacus, 407

Dipterocarpacea; , 1. 9

Dipterocarpus: 78

Dischidia, 510

Disperis, 836

Disporum, 866

Dittelasma, $55^{\circ}$

Dodonœe, 198

Dolichandrone, 580

Dolichos, 246, 81

Doodia, 1063

Doona, 80

Dopatrium, 556

Doritis, 804

Dorstenia, 751

Doryopteris, 1068

Dracæua, 864

Dregea, 509

Drosf ra, 284

Droseracex, 1). 32

Drymaria, 63

Drymoglossum, 1078

Drynaria, 1083

Dryopteris, 1042

Dumasia, $2333^{\circ}$

Dunbaria, 248

Duranta, 207

Durio, 32

Dysophylla, 6.33

Dysnxylun? 150
Ebenacea, p. 52

Ebermaiera, 586

Ecbolium, 609

Echinolytrum, 928

Eclipta, 429

Ehretia, 530

Elaphoglossum, 1084

Elatineæ, p. 8

Elatostema, 760

Eleiotis, 225

Eleocharis, 930

Elephantopus, 409

Elettaria, 848

Eleusine, 1005, 282

Elæagnaceæ, p. 77

Elæagnus, 689

Elæis, 271

Elæocarpus, 113

Elæodendron, 174

Ellipanthus, 210

Elytraria, 585

Elytrophorus, 1012

Embelia, 457

Emilia, 438

Enlıalus, 773

Enicostema, 523

Entada, 269

Enteropogon, 1001

Epaltes, 420

Epipogum, 831

Epithema, 577

Equisetaces, p. 114

Equisetum, 1033

Eragrostis, 1014

Eranthemum, 600

Eremochloa, 979

Eria, 789

Eriachne, 997 :

Ericacer, p. 50

Erigeron, 415, 139

Eriocaulon, 923

Eriocaulonaceæe, p. 100

Eriochloa, 947

Eriodendron, 99

Eriosema, 249

Erycibe, 537

Erythrina, 237, 77

Erythrospermum, 52

Erythroxylon, 116, 38

Eucalyptus, 110

Eugenia, 300, 11:

Eulophia, 797 
Euodia, 124

Euonymus, 169

Euphorbia, 697, 234

Euphorbiacers, p. 78, p. 14j

Furya. 76

Evolvulus, 544

Exacum, 520

Excœearia, 738

Fragræa, 517

Farmeria, 665

Fatsia, 13:3

Fergusonia, 369

Feronia, 136

Ficoideæ, p. 39

Ficus, 744,245

Filicium, 144

Fimbristylis, 927

Flacourtia, 53, 17

Flagellaria, 881

Flagellariaceæ, p. 96

Flemingia, 25I

Fleurya, 754

Floscopa, 880

Flueggea, 706

Fragaria, 103

Freycinetia, 895

Fuirena, 933

Furerea, 263

Gærtnera, 51?

Cialactia, 239

Galeola, 832

Galinsoga, 149

Galium, 405

Galphimia, 39

Garcinia, 70, 23

Gardenia, 382

Garnotia, 991

Gastrodia, 830

Gaultheria, 451

Gelonium, 735

Geniosporum, 626

Gentiana, 524

Gentianareæ, p. 58

Geodoru11, 798

Geophila, 399

Geraniacer, p. 14, p. 128

Geranium, 119

Gesneraceæ, p. 64, p. 142

Ginalloa, 693

Girardinia, 756

Gironniera, 743
Gisekia, 346

Givotia, 717

Gleichenia, 1087

Gleicheniacer, p. 12:

Gleniea, 193.

Gliricidia, 67

Globba, 839

Glochidion, 705

Gloriosa, 873

Glossocarya, 622

Glossogyne, 434

Glycine, 234

Glycosmis, 128

Glyptopetalum, 170

Gmelina, 619

Gnaphalium, 140

Gomphandra, 164

Gomphia, 141

Gomphocarpus, 174

Gomphrena, 220

Goniothalamus, 21

Goodenoviaceæ, p. 49

Goodyera, 824

Gordonia, 77

Gossypium, 30

Gouania, 186

Gracilea, 1009

Gramineæ, P. 105, p. 149

Grangea, 412

Grevillea, 233

Grewia, 110

Gluazuma, 36

Guettarda, 387

Guttiferæ, p. 8, p. 127

Gymnema, 505

Gymnopotalum, 324

Gymnopteris, Leptochilus, Hymenolepis

Gymnospermæ, p. 86, p. 150

Gymnosporia, 176

Gymnostachyum, 602

Gynandropsis, 43, 13

Gynostemma, 338

Gynura, 437

Gyrinops, 688

Gyrocarpus, 298

Habenaria, 835

Hæmodoraceæ, p. 93

Halophila, 775

Halopyium, 1015

Haloragiacoæ, p. 32

Harpullia, 197 
Hedychium, 842

Hedyotis, 370

Hedysarum, Desmodium

Helianthus, 146

Heliclurysum, 424, 141

Helicia, 684

Helicteres, 103

Heliotropinm, 534

Helminthostachys, 1095

Hemicyclia, 709

Hemidesmus, 494

Hemigyrosa, 190

Hemionitis, 1066

Hemitelia, 1039

Heptapleurum, 355

Heracleum, 353

Heritiera, 102

Hernandia, 683

Herpestis, 555

Hetæria, 820

Heterostemma, 512

Hevea, 236

Hewittia, 542

Heylandia, 212

Hibiscus, 96, 29

Hippocratea, 178

Hiptage, 117

Histiopteris, 1073

Holarrhena, 483

Holoptelea, 740

Holostemma, 502

Homalium, 321

Homonoia, 733

Hopea, 81

Hoppea, 521

Hortonia, 673

Hoya, 511

Hugonia, 115

Humata, 1050

Humboldtia, 266

Hunteria, 479

Hydnocarpus, 56

Hydrilla, 769

Hydrobryum, 664

Hydrocera, 123

Hydrocharitaceæ, ?. 64

Hydrocotyle, 347

Hydrolea, 528

Hydrophylax, 402

Hydrophyllaceæ, p. 5?

Hygrophila, 588

Hygrorhiza, 962
Hymenolepis, 1080

Hymenophyllaceæ, p. 115

Hymenophyllum, 1037

Hурөricaceæ, p. 8, p. 127

Hypericum, 69, 22

Hyplæne, 270

Hypolytrum, 940

Iehnanthrss, 950

Iclmocarpus, 493

Ilex, 168

Ilicinex, p. 19

Tlysantlıs, 560

Impations, 122

Imperata, 972

Incompletæ, p. 71, p. 143

Indigofera, 215,66

Ionidium, 49

Iphigenia, 872

Ipomæa, 541, 177

Ipsea, 794

Iridaceæ, p. 148

Isacline, 948

Isanthera, 578

Ischæmum, 978

Isoilema, 989

Isoetaceæ, p. 114

Isoetes, 1032

Isonandra, 462

Isotoma, 158

Ixora, 392

Jasminum, 469, 163

Jatropha, 715, 237

Josephia, 800

Julostylis, 94

Juncaceæ, p. 96

Juncus, 883

Jussiæa, 316

Justicia, 605

Ǩarlsura, 13

Kæmpf eria, 841, 25

Kalanchoe, 283

Kayea, 72

Kendrickia, 305

Kleinhovia, 34

Klugia, 576

Knoxia, 390

Kokoona, 172

Kurrimia, 177

Kyllinga, 926 


\section{$(159)$}

Labiatæ, p. 70, p. 143

Lactuca, 442, 156

Lagarosiphon, 770

Lagascea, 143

Lagenandra, 899, 120

Lagenophora, 414

Lagerstrœmia, 313

Laggera, 419

Landolphia, 166

Lantana, 612, 204

Laportea, 755

Lasiz, 909

Lasianthera, 163

Lasianthus, 400

Lasiosiphon, 686

Lastrea, Dryopteris, Polystichum

Launea, 443

Lauraceæ, p. 75, p. 145

Lawia, 661

Lawsonia, 312

Lecanthus, 758

Le日a, 188

Loersia, 961

Leguminosæ, p. 23, p. 130

Lemna, 912

Lemnaceæ, p. 99

Lentibulariaceæ, p. 63

Leonotis, 639

Lepidagathis, 603

Lepironia, 939

Leptadenia, 513

Leptaspis, 968

Leptochilus, 1046

Leptochloa, 1008

Lopturus, 1023

Lettsomia, 540

Leucæna, 97

Loucas, 638

Leucocodon, 376

Leucostegia, Davallia

Ligustrum, 472

Liliaceæ, p. 94, p. 148

Limacia, 31

Limnanthemum, 527

Limnophila, 554

Limnophytum, 916

Limonia, 132

Linaceæ, p. 14, p. 128

Lindera, 681

Lindsaya, 1056

Linociera, 470
Linum, 114

Liparis, 780

Lipocarpha, 934

Lippia, 613

Litchi, 56

Litobrochia, Pteris, Histiopteris

Litsea, 680

Lobelia, 446

Lobeliaceæ, p. 138

Loganiaceæ, p. 57

Lomaria, Blechnum

Lophatherum, 1018

Lopholepis, 966

Loranthaceæ, p. 77

Loranthus, 690

Lourea, 72

Loxococcus, 885

Ludwigia, 317

Luffa, 328

Luisia, 806

Lumnitzera, 296

Luvunga, 133

Lycopersicum, 179

Lycopodiacer, p. 116

Lycopodium, 1029

Lygodium, 1089

Lysimachia, 4.54

Lythracoæ, p. 36, p. 134

Maba, 466

Macaranga, 732

Machilus, 677

Mrrua, 44

Mrsa, 455

Magnoliaceæ, p. 3, p. 125

Mallotus, 730

Malpighiaceæ, p. 14, p. 128

Malvacer, p. 11, p. 127

Malvastrum, 26

Mangifora, 202, 58

Manihot, 241

Manisurus, 976

Mapania, 941

Mappia, 166

Maranta, 258

Marattia, 1092

Marattiaceac, p. 124

Mariscus, 925

Marsdenia, 506

Marsilea, 1035

Marsileaceæ, p. 115 
Martynia, 200

Mastixia, 357

Maurandia, 192

Medinilla, 307

Melampodium, 144

Molastoma, 304

Melastomaceæ, p. 34, p. 134

Melia, 146, 50

Meliacex, p. 17, p. 129

Molilotus, 64

Moliosma, 200

Melochia, 106

Melothria, 333

Memecylon, 308

Menisperma.ceæ, p. 4

Mentlia, 634, 213

Mesua, 73

Mezoneurum, 260

Michelia, 12, 1

Microcarpæa, 562

Microglossa, 416

Microlepia, 1052

Micromelum, 129

Microstylis, 779

Microtropis, 171

Mikania, 138

Miliusa, 24

Millingtonia, 197

Mimosa, 96

Mimusops, 465

Mirabilis, 216

Mischodon, 711

Mitrasacme, 516

Mitrephora, 22

Mnesithea, 977

Modecca, 322

Mohlana, 224

Mollugo, 345

Momordica, 326

Monimiaceæ, p. 75

Monochoria, 874

Monocotyledons, p. 86, p. 147

Monogramma, 1075

Monoporandra, 87

Monothecium, 604

Morinda 395

Moringa, 61

Moringacer, p. 130 .

Morus, 247

Moschosma, 627

Mucuna, 236

Mukia, 331
Mundulea, 21T

Munronia, 145

Murraya, 130

Musa, 852

Mussænda, 374

Myriactis, 413

Myriophyllum, 286

Myriostachya, 1013

Myristica, 672, 230

Myristicaceæ, p. 75, p. 145

Myrsinaceæ, p. 50, p. 139

Myrsine, 456

Myrtaceæ, p. 33, p. 134

Mystacidium, 812

Naiadaceæ, p. 99, p. 149

Najas, 920

Naravelia, 2

Nargedia, 382

Nasturtium, 40, 8

Naucles, 363

Nelumbium, 39

Nepenthacer, p. 74

Neponthes, 666

Nepholium, 195, 57

Nephrodium, Dryopteris

Nophrolopis, 1049

Noptunia, 268, 94

Nerium, 172

Neurocalyx, 367

Nicandra, 184

Nicotiana, 187

Nipa, 888

Niphobolus, Cyclophorus

Nothopegia, 205

Nothosærua, 65

Notonia, 439

Notothixos, 692

Nyctaginacea, p. 71, p. 143

Nyctanthes, 164

Nymphra, 38

Nymphæaсељ, p. 5

Oberonia, 778

Ochlandra, 1028

Ochna, 140

Ochnaceæ, p. 17

Ochrosia, 48 I

Ocimum, 625், 210

Octarrhena, 818

Odina, 203

Odontosoria, 1053 
Enothera, 115

Olacineæ, p. 18

Olax, 159

Oldenlandia, 371,135

Olea, 471

Oleaceæ, p. 53, p. 139

Oloandra, 1047

Onagraceæ, p. 37, p. 135

Oncosperma, 886

Ophioglossaceæ, p. 124

Ophioglossum, 1093

Ophiopogon, 853

Ophiorrhiza, 373

Ophioxylon, Rauvolfia

Opilia, 161

Oplismenus, 954

Opuntia, 126

Orchidacere, p. 86, p. 147

Oreodoxa, 269

Ormocarpum, 224

Orobanchaceæ, p. 63

Oropetium, 1000

Orophea, 25

Oroxylum, 579

Orthosiphon, 628

Oryza, 960

Osbeckia, 303

Osmelia, 320

Osmunda, 1090

Osmundaceæ, p. 124

Ostodes, 719

Ottelia, 772

Oxalis, 120,41

Oxystelma, 498

Oxytenanthera, 1026

Pachygone, 33

Palaquium, 464

Palmaceæ, p. 96, p. 149

Panax, 132

Pancratium, 857

Pandanacex, p. 97, p. 149

Pandanus, 894, 272

Panicum, 949

Papaveraceæ, p. 125

Paramignya, 134

Parkeriaceæ, p. 123

Parkia, 93

Parkinsonia, $8 \%$

Parochetus, 2 ] 4

Parsonsia, 486

Paspalum, 946
Passiflora, 117

Passifloraceæ, p. 37, p. 135

Pavotta, 393

Pavonia, 93

Podaliaceæ, p. $65, p .145$

Podalium, 582

Pedicularis, 567

Pollæa, 1067

Pellionia, 759

Peltophorum, 259

Pemphis, 311

Pennisetum, 955

Pentapetes, 105

Pentatropis, 500

Peperomia, 670, 229

Peplidium, 563

Peretis, 967

Periandra, 76

Pericopsis, 257

Persea, 232

Peucedanum, 352, 129

Phajus, 795

Phaleria, 687

Phaseolus, 243, 78

Phaylopsis, 591

Phegopteris, Dryopteris

Phœnix, 889

Pholidota, 786

Phormium, 265

Photinia, 281

Phragmites, 1011

Phreatia, 817

Phrynium, 850

Phyllanthus, 704, 235

Phyllochlamys, 749

Physalis, 549, 182

Physurus, 822

Phytolacca, 225

Phytolaccacex, p. 144

Piləa, 757, 251

Pimpinolla, 351

Piper, 669

Piperacece, p. 74, p. 145

Pisonia, 643,218

Pistia, 897

Pisum, 74

Pithocolobium, 274, 100

Pittosporacex, p. 7

Pittosporum, 57

Pityranthe, 108

Plantaginacex, p. 71, p. 14.3

Plantago, 641, 215 
Plecospermum, 753

Plectranthus, 629,211

Pleocnemia, Aspidium

Pleopeltis, Polypodium, Drynaria

Pleurostylia, 173

Plumbaginacese, p. 50, p. $13 \delta$

Plumbago, 453, 159

Plumeria, 168

Poa, 1021

Podadenia, 728

Podochilus, 816

Podostemaceæ, p. 73

Podostemon, 663

Pogonatherum, 980

Pogonia, 834

Pogostemon, 632

Poinciana, 86

Pollia, 870

Pollinia, 974

Polyalthia, 18

Polybotrya, 1045

Polycarpæa, 65

Polycarpon, 64

Polygala, 58

Polygalaceæ, p. 7

Polygonaceæ, p. 73, p. 144

Polygonum, 660, 226

Polypodiaceæ, p. 116

Polypodium, 1081

Polypogon, 993

Polyscias, 354

Polystachya, 801

Polystichum, 1044

Polytoca, 970

Pometia, 196

Pommeroulla, 1010

Pongamia, 254

Pontederiaceæ, p. 95

Porana, 178

Portulaca, 66

Portulacaceæ, p. 8, p. 126

Potamogeton, 918

Potentilla, 277

Poterium, 279

Pothos, 910

Pouzolzia, 764

Promna, 618

Primulaceæ, p. 50, p. 139

Prismatomeris, 396

Priva, 616

Procris, 761

Prosaptia, Davallia
Proteaceæ, p. 76, p. 145

Prunus, 101

Psoudanthistiria, 987

Psoudarthria, 227

Pseudocarapa, 151

Psidium, 111

Psilotaceæ, p. 114

Psilotrichum, 650

Psilotum, 1030

Psophocarpus, 79

Psoralea, 216

Psychotria, 397

Pteridium, 1074

Pteris, 1072

Pterocarpus, 253, 84

Pterospermum, 104

Ptyssiglottis, 608

Punica, 114

Pupalia, 649

Putranjiva, 708

Pycnospora, 226

Pycreus, Cyporus

Pygeum, 275

Pyrenacantha, 167

Pyrus, 105

Quisqualis, 109

Randia, 381

Ranunculaceæ, p. 1

Ranunculus, 5

Raphanus, 12

Rauvolfia, 477

Reinwardtia, $3 \pi$

Remirea, 938

Remusatia, 905

Rhabdia, 532

Rhamnaceæ, p. 20

Rhamnus, 182

Rhaphidophora, 908

Rhinacanthus, 607

Rhipsalis, 342

Rhizophora, 288

Rhizophoraceæ, p. 32

Rhododendron, 452

Rhodomyrtus, 299

Rhynchocarpa, 334

Rhynchoglossum, 196

Rhynchosia, 250

Rhynchospora, 936

Rhynchostylis, 803

Richardia, 273 
Ricinus, 243

Rivea, 538

Rivina, 223

Rosa, 104

Rosaceæ, p. 31, p. 133

Rothia, 211

Rottbœllia, 975

Rotilera, Mallotus

Rourea, 208

Roxburghiaceæ, p. 94

Rubia, 404

Rubiaceæ, p. 41, p. 137

Rubus, 276

Ruellia, 590

Rumex, 227

Rungia, 610

Ruppia, 919

Rutaceæ, p. 15, p. 128

Sabiaceæ, p. 22

Saccharum, 973, 27y

Saccolabium, 809

Sageretia, 184

Sagina, 19

Salacia, 179

Salicornia, 657

Salomonia, 59

Salvadora, 473

Salvadoraceæ, p. 54

Salvia, 214

Salviniaceæ, p. 115

Samadora, 138

Samydaceæ, p. 59

Sanicula, 348

Sansevieria, 854

Santalaceæ, p. 78

Sapindaceæ, p. 21, p. 130

Sapindus, 194

Sapium, 737, 244

Sapotaceæ, p. 51, p. 139

Saprosma, 401

Saraca, 265

Sarcanthus, 810

Sarcocophalus, 359

Sarcochilus, 802

Sarcococca, 698

Sarcostemma, 504

Satyrium, 837

Sauropus, 703

Saxifragaceæ, p. 31, p. 133

Scævola, 445

Schizæz, 1088
Schizæaceæ, p. 123

Schizoloma, 1055

Schizostigma, 378

Schleichera, 192

Schumachoria, 9

Sciaphila, 914

Scilla, 871

Scirpodendron, 942

Scirpus, 931

Scitamineæ, p. 92

Scloria, 943

Scleropyrum, 695

Scolopia, 51

Scoparia, 194

Scrophulariaceæ, p. 62, p. 131

Scutellaria, 636

Scutia, 183

Scyphiphora, 386

Scyphostachys, 384

Sebastiania, 739

Secamono, 496

Sechium, 125

Solaginella, 1031

Selaginellacer, p. 114

Semocarpus, 204

Senecio, 440

Serpicula, 285

Sesamum, 583, 201

Sesbania, 219, 68

Sesuvium, 343

Sotaria, 951,280

Shorea, 79

Shutoria, 232

Sida, 89

Sideroxylon, 461

Siegesbeckia, 428

Simarubaceæ, p. 16, p. 129

Smilax, 862

Smithia, 222

Solanaceæ, p. 61, p. 140

Solanum, 5 18,180

Sonchus, 157

Sonerila, 306

Sonneratia, 314

Sophora, 256

Sopubia, 565

Sorghum, 278

Spathodea, 198

Spergula, 20

Spermacoco, 403

Sphæranthus, 421

Sphærocaryun, 992 
Sphenoclea, 448

Spilanthes, 432

Spinifex, 958

Spiræa, 102

Spiranthes, 826

Spondias, 207, 60

Sporobolus, 994

Stachytarpheta, 615, 20.5

Stellaria, 62, 18

Stemodia, 193

Stemona, 861

Stemonoporus, 86

Stenochlæna, 1062

Stenoloma, Odontosoria

Stenosiphonium, 593

Stenotaphrum, 956

Stephania, 34

Stephegyne, 362

Sterculia, 101

Sterculiaceæ, p. 12, p. 128

Stereospermum, 581, 1.99

Streblus, 750

Streptogyne, 1017

Striga, 564

Strobilanth $f$, 594

Strombosia, 160

Strongylodon, 238

Strychnos, 518

Stylidiaceæ, p. 49

Stylidium, 444

Stylosanthes, 221

Styraceæ, p. 53

Suæda, 658

Sunaptea, 82

Suriana, 139

Susum, 882

Swertia, 526

Swi. teniฉ, 52

Symphorema, 623

Symplocos, 468

Synantherias, 904

Synedrella, 147

Syngramme, Coniogramme

Syzygium, Eugenia

Tabernæmontana, 484, 170

Tacea, 858

Taccaceæ, p. 94

Tænitis, 1079

Tæniopliyllum, 814

Tagetes, 151

Tainia, 791
Talinum, 21

Tamarindus, 91

Tamariscineæ, p. 8

Tamarix, 67

Taraxacum, 155

Taxotrophis, 748

Tectona, 208

Teinostachyum, 1027

Tephrosia, 218

Teramnus, 235

Terminalia, 294, 108

Ternstrœmia, 74

Ternstrœmiaceæ, p. 9, p. 130

Tetracera, 7

Tetrameles, 341

Tetranthera, Litsea

Teucrium, 640

Thalassia, 774

Thalictrum, 4

Theriophonum, 902

Thea, 24

Theobroma, 35

Thespesia, 97

Thismia, 777

Thuarea, 957

Thunbergia, 584, 202

Thymelæa.ceæ, p. 76

Tibouchina, 113

Tiliaceæ, p. 13

Tiliacora, 30

Timonius, 388

Tinospora, 27

Tithonia, 145

Toddalia, 126

Torenia, 538

Tournefortia, 533

Toxocarpus, 497

Trachys, 963

Tragia, 727

Tragus, 964

Trapa, 318

Trema, 742

Trewia, 726

Trianthema, 344

Tribulus, 118

Trichadenia, 55

Trichodesma, 535

Trichomanes, 1036

Trichopus, 860

Trichosanthes, 323, 119

Tridax, 150

Trifolium, 65 
Trigonostemon, 718

Triphasia, 44

Tripogon, 1002

Triumfetta, 111

Triuridaseæ, p. 99

Tropæolum, 40

Tropidia, 828

Turnera, 116

Turneraceæ, $p .13 .5$

Turpinia, 199

Tylophora, 587

Typha, 896

Typhaceæ, p. 98

Ulex, 63

Umbelliferæ, p. 40, p. 136

Uncaria, 364

Unona, 17, 4

Uraria, 228, 71

Urena, 92

Urginea, 870

Urophyllum, 377

Urostigma, Ficus

Urticaceæ, p. 83, p. 146

Utricularia, 571

Uvaria, 2

Vacciniaceæ, p. 50

Vaccinium, 450

Vahlia, 282

Vallaris, 487, 171

Vanda, 807

Vandellia, 559

Vanilla, 829, 253

Vascular Cryptogams, p. 92

Vateria, 85

Vatica, 83

Ventilago, 180

Verbascum, 190

Verbena, 206

Verbenaceæ, p. 68, p. 142

Vernonia, 408

Veronica, 195

Vetiveria, 985

Viburnum, 358

Vicos, 425
Vigna, 244, 80

Villebrunea, 765

Vinca, $482,16 \%$

Viola, 48, 14

Violaceæ, p. 6, p. 126

Viscum, 691

Vitex, 620

Vitis, 187,54

Vittaria, 1076

Wahlenbergia. 447

Walsura, 153

Waltheria, 107

Wobera, 379

Websteria, 932

Wedelia, 431

Weihea, 292

Wendlandia, 365

Wikstrœmia, 685

Willughbeia, 475

Wissadula, 91, 28

Withania, 550

Wolffia, 913

Woodfordia, 310

Wormia, 10

Wrightia, 488

Xanthium, 427

Xanthophyllum, 60

Ximenia, 158

Xyridaceæ, p. 95

Xyris, 875

Zanonia, 339

Zanthoxylum, 1'25, 43

Zea, 276

Zehneria, 332

Zenkeria, 998

Zeuxine, 825

Zingiber, 846, 256

Zingiberaceæ, p. 147

Zizyphus, 181

Zornia, 220

Zoysia, 965

Zygophyllacer, p. 14 


\section{SINHALESE NAMES.}

A number of prefixes, dec., occur constantly throughout this list, and the translations are given here for reference :-

\begin{tabular}{lll|lll} 
Alu & $\ldots$ & ash & Katu & $\ldots$ & thorny \\
Amba & $\ldots$ & mango & Kiri & $\ldots$ & milk \\
Bata & $\ldots$ & reed & Kudu & $\ldots$ & dust \\
Bin & $\ldots$ & ground & Lunu & $\ldots$ & salt \\
Bu & $\ldots$ & woolly & Ma, Maha & $\ldots$ & large \\
Dada & $\ldots$ & ringworm & Mal & $\ldots$ & flower \\
Divi & $\ldots$ & tiger & Mediya & $\ldots$ & frog \\
Diya & $\ldots$ & water & Mudu & $\ldots$ & sea \\
Dodan & $\ldots$ & orange & Nil & $\ldots$ & blue, greon \\
Dunu & $\ldots$ & bow & Panu & $\ldots$ & worm \\
Ela & $\ldots$ & pale & Poni & $\ldots$ & swoet \\
Et & $\ldots$ & groat & Pini & $\ldots$ & dew \\
Eta & $\ldots$ & soed & Potu & $\ldots$ & bark \\
Gal & $\ldots$ & rock & Rana & $\ldots$ & golden \\
Gan & $\ldots$ & rivor & Rata & $\ldots$ & foreign \\
Gas & $\ldots$ & tree & Rat, ratu & $\ldots$ & red \\
Geta & $\ldots$ & joint & Sudu & $\ldots$ & white \\
Goda & $\ldots$ & land & Tel & $\ldots$ & oil \\
Gon & $\ldots$ & bullock & Titta & $\ldots$ & bitter \\
Hal & $\ldots$ & rice & Uru & $\ldots$ & pig \\
Hin & $\ldots$ & small & Wal & $\ldots$ & wild \\
Kaha & $\ldots$ & yollow & Wel & $\ldots$ & climber \\
Kalu & $\ldots$ & black & Weli & $\ldots$ & sand \\
Kara & $\ldots$ & rough & Yak & $\ldots$ & devil \\
& & & & &
\end{tabular}

Aba, 13

Achariya-pala, 607

Agal-adara, 1607

Aga-mula-neti-wel, 1434

Agu-karni, 1068

$\mathrm{Ahu}, 1050,1051$

Akkapana, 144

Akmediya, 1964

Akmella, 1145

Alan, 2210

Alandu, 1995

Alanga, 1402

Alariya, 233

Alu, 2210

Alu-bo, 770

Alu-gas, 2210

Alu-pila, 552

Alu-puhul, 175

Amba, 74

Amba-kaha, 2204

Amba-wila. 1452

Amu, 2569
Amukkara, 1446

Andara, 695

Andun-wenna, 409

Angana, 1025

Anitta, 1608

Ankenda, 347

Annasi, 355

Anoda, 233, 235, 6

Aralu, 746

Aramana, 678

Aridda, 500

As-wel, 1295

Aswenna, 573

Ati-udayan, 2345

Attana, 1447

Attikka, 1986

Atulzotiya, 44

Badal-wanassa, 2816

Badulla, 491, 493, 496

Baka-muna-miris, 1744

Baka-muna-tana, 2533 
Bakmi, 949

Bala, 499

Bala-nakuta, 1619

Ba-loliya, 582

Balu-dan, 1191, 223

Balu-nakuta, 393

Bambara-wel, 650

Bandura-wel, 1737

Barawa-embilla, 1896

Bata-damba, 779

Bata-kirilla, 309

Batala, 243

Bata-li, 2808

Batu-karivila, 890

Bedi-del, 1989

Beli, 60

Beli-patta, 257

Beraliya, 194

Beriya, 750

Beru, 1922

Beru-diyanilla, 872

Bevila, 227

Bilin, 53

Bim-pol, 2251

Bin-beru, 10

Bin-dada-kiriya, 1837

Bindara, 1353

Bin-karal-heba, 1699

Bin-kohomba, 379

Bin-me, 619

Bin-nuga, 1320

Bin-olu, 1368

Bin-sawan, 1463

Bin-siyambala, 681, 682

Bin-tamburu, 1421

Bo, 341

Bodi, 548

Bo-kera, 371, 373

Bol-hinda, 2298

Bolila, 1662

Bolvila, 1662

Bombi, 1780

Bombu, 1239

Bomi, 1780

Bonchi, 99

Bora-daminiya, 284

Boralu, 1178

Boru-pan, 2493

Bowitiya, 805, 808

Bu-dada-kiriya, 1836

Bu-embilla, 1901

Bu-getiya, 306
Bu-hore, 178

Bu-hunu-kirilla, 1884

Bu-katu-kenda, 1936

Bu-kenda, 1936

Bu-kinda, 64

Bu-kobbe, 466

Bulat, 1742

Bulat-wel, 1742

Bulu, 745

Bulu-mora, 476

Bil-me, 621

Bu-nelu, 1539

Bu-nuga, 1968

Bu-pila, 556

Burulla, 460

Buruta, 392

Bu-seru, 1625

Bu-tora, 680

But-sarana, 2234

Bu-wal-anguna, 1292

Caju, 75

Chanchala, 596

Chocolat-gas, 45

Dada-kaha, 2200

Dada-kehel, 2364

Daluk, 1830

Damala, 99

Dambu, 766

Daminiya, 281

Dan, 767

Daradamala, 101

Dara-wetakolu, 896

Datketiya, 46, 996

Dawata, 741

Dawu, 749

Dawul-kurundı, 1790

Dedikaha, 855

Dehi, 59

Del, 1989

Delun, 158

Demata, 1630

Desa-ala, 375

Devadaram, 308

Dik-w€nna, 275

Divi-adiya, 1409

Divi-kaduru, 1284

Divi-pahuru, 1409

Diwul, 366

Diya-beru, 1921

Diya-danga, 1519 
Diya-embul-embiliya, 2837

Diya-habarala, 2271

Diya-hawari, 2027

Diya-kirilla, 1370

Diya-kirindi-wel, 423

Diya-kudalu, 343

Diya-labu, 172

Diya-meneriya, 2279

Diya-midella, 801

Diya-mitta, 75

Diya-na, 170

Diya-nidi-kumba, 691

Diya-nilla, 1514

Diya-panshi, 2371

Diya-para, 20

Diya-parandella, 2338

Diya-pasj, 1497

Diya-ratambala, 686

Diya-ratmal, 686

Diya-siyambala, 564

Diya-taleya, 945

Diya-tana-kola, 2593

Diya-wawul-etiya, 666

Dodan-kaha, 856

Dodan-pana, 348

Dodan-wenna, 855

Domba, 160

Dombakina, 157

Dorana, 181

Dotalu, 2313

Duhudu, 419

Dul, 1295

Dummella, 886

Dun, 188

Dunu-keyiya, 2334

Dunu-madala, 1520

Dutu-satutu, 1484

Ehela, 671

Ehotu, 1978

Eka-weriya, 1276

Ela-batu, 1443

Ela-dada-kiriya, 1835

Fla-golkatu, 156

Ela-imbul, 19

Ela-kuru-tana, 2778

Ela-mal, 2206

Ela-midella, 803

Ela-netul, 1181

Ela-nuga, 1978

Ela-palol, 276

Ela-wel, 2323
Elbudda, 481

Embarella, 501

Embul-balkmi, 950

Endaru, 336

Ensal, 2230

Epala, 289

Erabadu, 608

Et-adi, 1102

Etæ-hirilla, 479

Eta-kirindi-wel, 1344

Et-amba, 484

Etambiriya, 1389

Etamburu, 1338

Eta-miriya, 1389

Eta-mura, 400

Eta-pan, 2527

Eta-timbiri, 1228

Eta-werella, 478

Eta-wira, 1892

Et-bemi-kiriya, 2233

Et-demata, 1629

Et-heraliya, 422

Et-honda, 1385

Et-korasa-wel, 9

Et-kukuruman, 1010

Et-nerenchi, 1521

Et-olu, 78

Etora, 2615

Et-pamba, 3063

Et-pitawakka, 1850

Et-setiya, 1385

Et-toriya, 351

Et-tora, 631

Et-tuttiri, 2729

Etuna, 289

Et-undupiyali, 591

Gahala, 2360

Gal-ambala, 910

Galangal, 352

Gal-demata, 328

Gal-ehi, 2431

Galis, 1015

Gal-kapura-walliya, 1660

Gal-karanda, 687, 1031

Gal-kehel, 2235

Gal-kura, 273

Gal-mendora, 683

Gal-mora, 476, 1762

Gal-ota, 1948

Gal-siyambala, 684

Gal-weralu, 302 
Gal-wira, 1892

Gammalu, 653

Gam-miris-wel, 1744

Ganda-pana, 405, 283

Gan-kollan-kola, 1663

Gan-mi, 1203

Garandi-kidaran, 2244

Gas-bevila, 230

Gas-dul, 2020

Gas-gonika, 569

Gas-kahambiliya, 2000

Gas-karal-lioba, 1709

Gas-kayila, 1887

Gas-kola, 612

Gas-keppitiya, 1909

Gas-kotala, 1464

Gas-notul, 1966

Gas-nidikumba, 317

Gas-pinna, 1638

Gas-tala, 1645

Gedumba, 1963

Gendakola, 142

Geriata, 1265

Geta-kaha, 798

Geta-kola, 982

Geta-notul, 1993

Geta-oluwa, 2231

Geta-pichcha, 225

Geta-tumba, 1679, 1681

Gini-hiriya, 1353

Gin-pol, 2316

Girapala, 2278, 2280

Giritilla, 1393

Giriwadi-bevila, 228

Goda-linguru, 704

Goda-kaduru, 1345

Goda-karawu, 2543

Goda-kirilla, 1960

Goda-midella, 802

Goda-para, 22

Goda-wawul-otiya, 670

Go-hiri, 2412

Go-jabba, 2653

Gokatu, 153

Golu-mora, 714

Gomma, 121

Gona, 1233

Gona-ala, 2249

Gonapana, 156, 386

Gona-wol, 226

Goll-gotu, 1992

Gon-kaduru, 1280

Gon-kekiri, 894

Gopalanga, 905

Goradiya, 602

Goraka, 152

Goyi-wol, 2307

Gurenda, 1961

Guru-kina, 158

Gurulla, 460

Guruwal, 2571

Habara, 1219

Habarala, 2362

Halk-ambala, 914

Hakan, 1587

Hal, 85

Hal-bembiya, 282

Hal-mendora, 205, 209

Hal-milla, 276

Halpan, 2426, 2475

Hambu-pan, 2337

Hamparila, 1943

Hampella, 579

Hampinna, 647

Hana, 524

Han-palanda, 747

Haran-kaha, 2201

Hata-wariya, 2256, 2258

Hawari-madu, 1412

Hodawalka, 1952, 1954

Hedolka, 1952, 1954

Hekarilla, 1438

Helamba, 953

Hemanella, 1667

Heri-mena-dotta, 1617

Hewan-pan, 2432

Hilk, 485

Hima, 755

Himbutu-wol, 427

Hin-ambala, 1367

Hin-anoda, 232

Hin-bin-kohomba, 1584

Hin-bin-tal, 2239

Hin-botiya, 1099

Hin-dan, 767

Hin-ombilla, 1903

Hin-embul-ombiliya, 316

Hin-epala, 240

Hin-eraminiya, 431

Hin-genda-kola, 144

Hin-gota-kola, 1084

Hin-gotu-kola, 930

Hingul, 387 
Hinguru, 703

Hinguru-piyali, 349

Hin-himbutu-wel, 426

Hin-kabarasa, 2254

Hin-kadol, 1197

Hin-karamba, 1275

Hin-katu-pila, 1885

Hin-kebella, 1897

Hin-kekiri, 899

Hin-keyiya, 2532

Hin-kina, 158

Hin-kokmota, 2404

Hin-kuretiya, 848

Hin-madu, 1411

Hin-mottu, 1445

Hin-muda-mahana, 1123

Hin-napiritta, 246

Hin-pala, 922

Hin-pamba, 3065

Hin-sarana, 918

Hin-takkada, 1169

Hin-tala, 1642

Hin-tambala, 1377

Hin-tolabo, 2241

Hin-undupiyali, 593

Hiressa, 443

Hiritala, 2247

Ho-medíriya, 1216

Honda-beraliya, 198

Hondala, 883

Hondapara, 21

Hora, 179

Hulan-hik, 391

Hulan-kiriya, 2232

Hulan-mara, 707

Hulan-tala, 195

Hunu-kirilla, 1875

Huriyi, 706

Ikili, 1570

Ikiliya, 876

Illa, 1621

Illuk, 2670

Imbul, 261

Indi, 2317

Induru, 2308

Ingini, 1346

Inguru, 351

Innala, 291

Ipetta, 31

Iramusu, 1298

Iringu, 377
Iri-weriya, 290

Iriya, 1759

Iru-raja, 2166

Itana, 2709

Itta, 940

Itta-wel, 940

Jambola, 58

Jambu, 154

Jata-makuta, 2065

Jayapala, 329

Kabal-mara, 707

Kabarasa, 2254

Kadala, 92

Kadalu-kola, 325

Kadol, 735, 736

Kadumboriya, 1225, 1227 , $1234,1235,1236$

Kadupara, 1153

Kaduru-ketiya-wel, 753

Kaha, 20, 350

Kaha-andana-hiriya, 521

Kaha-gona-kola, 1450

Kaha-kala, 1222

Kaha-penela, 469

Kaha-petan, 688

Kahata, 804

Kahata-kondol, 362

Kaha-tel-kola, 1414

Kaju, 75

Kakuru, 433

Kalaha, 1979

Kalanduru, 2441

Kalati, 40

Kalatiya, 711

Kala-wel, 655, 657

Kalinda, 1283

Kallu, 1227

Kalu-alanga, 1402

Kalu-badulla, 489

Kalu-habaraliya, 1218

Kalu-kadumberiya, 1435

Kalu-kan-weriya, 1435

Kalu-kera, 47

Kalu-maduwa, 1974

Kalu-mediriya, 1229, 1234

Kalu-wala, 352

Kalu-wara, 1223

Kalu-waraniya, 1601

Kalu-wella, 1222, 1236, 1237

Kamaranga, 52 
Kampotta, 1895

Kana-bakmi, 949

Kana-gona, 1990

Kana-goraka, 153

Kiandala, 2360

Kiandul-essa, 729

Kiankumbala, 479, 1307

Kankumbal-ketiya, 714

Kankun, 1420

Kapu-kinissa, 255, 256

Kapura, 1452

Kapuru, 50

Kapuru-walliya, 292

Kaputu-bo, 1975

Kara, 1037

Karal-iríngu, 379

Karan, 1380

Karapincha, 353

Karawala-kebella, 1902

Kiarawata-mana, 2725

Karawu, 1873

Karivila, 890

Karon-damba, 769

Kasa, 347

Kata-rodu-wel, 626

Katu-andara, 700

Katu-anoda, 5

Katu-boda, 262

Katu-embilla, 1800

Katu-ikili, 1570

Katu-ikiri, 1532

Katu-imbul, 260

Katu-karandu, 1571

Katu-kenda, 112

Katu-kina, 54

Katu-kiriya, 353

Katu-kitul, 2314

Katu-kukul-ala, 363

Katu-kurundu, 113

Katu-nelu, 1572

Katu-patuk, 180

Katuru-murunga, 87

Katu-tampala, 1693

Katu-timbol, 1996

Katu-una, 2803

Katu-wala, 2246

Katu-wel-batu, 1443

Kaudu-bo, 1975

Kawalu, 2626

Kawudu-kekiri, 902

Kebella, 1896

Kehel, 2235
Keli-pittan, 76

Kekala, 31

Kekatiya, 2380

Kelkilla, 3061

Kekiri, 894

Kekiri-wara, 17, 871

Keku, 57

Kekuna, 377

Keleniya, 2210

Keliya, 285

Kenda, 1947

Ken-henda, 1637

Keppitiya, 1909

Kere-koku, 3059

Kesi-pissan, 76

Keta-kala, 1840

Ketambilla, 118

İetiya, 124, 714

Kevitiya-kera, 832

Kidaran, 2356

Kikirindi, 1411, 2661

Kina, 161, 167

Kinahiriya, 19

Kiri-anguna, 1323

Kiri-badu, 1399

Kiri-gedi, 1273

Kiri-hømbiliya, 1207

Kiri-henda, 1688

Kiri-hiriya, 1207

Kiri-kaju, 238

Kiri-kon, 389

Kiri-kondol, 360

Kirilla, 870

Kiri-madu, 1416

Kiri-makulu, 1955

Kiri-mawara, 1283

Kiri-misastru, 2618

Kirindi-wel, 502

Kiri-pella, 1977

Kiri-walla, 1283

Kiri-warala, 1200

Kiri-wel, 1052, 1273, 1297

Kitul, 2315

Kobbe, 466

Kobo-mal, 779

Kobo-mella, 1101

Kolnila, 2365

Kolıomba, 381

Kohu-kirilla, 285

Kokatiya, 153, 155

Kokmota, 2396

Kokun, 416 
Kolikara-mal, 1488

Kollu, 103

Kolon, 951

Komadu, 174

Kon, 467

Kopi, 193

Kora-kaha, 844

Korasa-wel, 8

Kos, 344

Kosatta, 1918

Kosbada, 1779

Kosgona, 1970

Kota-dimbula, 1983

Kotala-wel, 1465

Kotikan-beraliya, 191

Kotikan-bevila, 231

Kottala-himbutu, 427

Kottamba, 145

Kowakka, 889

Kudalu-dehi, 56

Kudalu-kola, 325

Kudalu-mal, 331

Kudu-dawula, 1790

Kudu-hedaya, 2813

Kudu-kurundu, 1768

Kudu-miris, 346

Kukula-wel, 2325, 2327

Kukul-man, 479

Kukulu-pala, 139

Kukuruman, 1011

Kulu-liyan, 1874

Kumatiya, 1691

Kumbalu, 367

Kumbuk, 748

Kumburu-wel, 665

Kunumella, 1219

Kuppa-meniya, 1926

Kurakkan, 383

Kura-tampala, 1696

Kurotiya, 848

Kurinnan, 1311

Kurundu, 1765

Landesi, 301

Landittan, 1779

Lankenda, 2205

Lawulu, 1198

Layu, 2652

Lena-batu, 2715

Len-teri, 2312

Lima-dehi, 56

Liniya, 270
Liyan, 88I

Liyangu, 881

Lolu, 1371

Lovi-lovi, 21

Lunu-ankenda, 344

Lunu-dan, 1190

Lunu-ketiya-wel, 74

Lunu-madala, 1520

Lunu-midella, 380

Lunu-warana, 91

Lunu-wila, 1459

Ma-banda, 1391

Ma-bin-tal, 2238

Madan, 780

Madara, 1843

Madatiya, 693

Madol, 154

Madu, 2023

Madul-karanda, 654

Madurutala, 1643

Maha-ambala, 1366

Maha-andara, 700

Maha-badulla, 487

Maha-beru, 1921

Maha-bowitiya, 814

Maha-bulu-mora, 376

Maha-dan, 780

Maha-debara, 430

Maha-diya-dul, 2011

Maha-diya-siyambala, 565

Maha-dumudu, 241

Maha-epala, 251

Maha-eraminiya, 434

Maha-geta-pan, 2507

Maha-getiya, 306

Maha-gotukola, 928

Maha-hedaya, 2814

Maha-kabarasa, 2255

Maha-karamba, 1274

Maha-kiri-wel, 1052

Maha-kuretiya, 764

Maha-madu, 1416, 2024

Maha-midi, 1627

Maha-nuga, 1967

Maha-pamba, 3064, 3065

Maha-pengiri, 2716

Maha-ratambala, 1040

Maha-ratmal, 1180

Maha-rawana-rowula, 2640

Maha-sarana, 920

Maha-tawara, 945 
Maha-undupiyali, 594

Maha-yak-wanassa, 1682

Makulu, 120

Malabatu, 1440

Malaboda, 1756

Malalabu, 1749

Mala-miris-wel, 1748

Mal-otora-tana, 2780

Malitta, 864

Mal-kera, 370

Mal-mora, 186, 199

Mana, 2717

Manda-madini-wol, 1085

Mangus, 28

Manyokka, 331

Mapat-kebella, 1895

Mapat-kina, 157

Mara, 705

Maran, 761

Maranda, 761

Ma-ratmal, 1180

Mas-bedde, 1309

Mas-mora, 694

Mata-bimbiya, 1184

Maussa, 1999

Ma-wewel, 2328

Mayani, 1604

Mayella, 689

Mayuru-tana, 2758

Meda-hangu, 1305

Meditella, 1962

Modiya, 1279

Mediya-jawale, 2034

Mediy»-wel, 456

Mehi-wal, 2534

Me-karal, 102

Molla, 397

Mella-dum-kola, 1851

Mondora, 204

Moneri, 2610, 2612

Meni-damba, 780

Mi, 1201

Midi, 1624

Migon-karapinchæ, 354

Mihiriya, 1207

Milla, 1633

Milla-kunari, 1908

Mimini-mara, 712

Miris, 1744

Miwana-kola, 2959

Miwonna, 39

Miyan-milla, 1633
Molpedda, 1200, 1206, 1207

Molpotta, 389

Monara-kudimbiya, 1092

Monara-petan, 2261

Mora, 474

Mottu, 1445

Mottu-tana, 2456

Muda-mahana, 1125

Mudilla, 800

Mudu-awara, 614

Mudu-bin-nuga, 1321

Mudu-bin-tamburu, 1424

Mudu-dada-kiriya, 1833

Mudu-өtora, 2742

Mudu-geta-kola, 1081

Mudu-halpan, 2474

Mudu-kaduru, 1281

Mudu-kalanduru, 2420

Mudu-keyiya, 2332

Mudu-murunga, 661

Mugunu, 915

Mukunu-wenna, 1712

Mun, 621

Muna-mal, 1213

Mun-ota, 621

Murunga, 77

Muruta, 867

Muruwa-dul, 1313

Mussenda, 1002

Muwa-kiriya, 1308

$\mathrm{Na}, 169$

Naha, 1797

Na-imbul, 476, 477

Nala-gas, 2772

Na-mondora, 202

Na-piritta, 247

Nara-wel, 1, 3, 2324

Nava, 266

Nawr-handi, 323

Nayi-miris, 258

Nebedda, $163 !$

Nedun, 664

Nelli, 1857

Nelu, 1539 to 1567

Nelun, 80

Neralu, 418

Notawu, 44

Neya-dasso, 174

Nidi-kumba, 128

Nigunu, 915

Nika, 1632 
Nika-damulu, 482

Nil-andana-hiriya, 522

Nil-awari, 544

Nil-gona-kola, 1451

Nil-katarodu, 626

Nil-me, 102

Nil-monaressa, 1499, 1501

Nil-nika, 1632

Nil-pichcha. 1024

Nil-puruk, 1535

Niri-wel, 31

Niviti, 1719

Niyanda, 2237

Niyangala, 2270

Niřan-meta-kolu, 895

Nuga, 1970

Odu-talan, 1669

O-keyiya, 2333

Okuru, 1944

Olindawel, 598

Olu, 78, 1366

Olu-petts, 1917

Omara, 37

Ota, 1948

Otala, 1645

Palala, 135

Palanga, 30

Palatu, 1794

Palen, 422

Palol, 276

Palu, 1214

Palukan, 30

Pamburu, 365

Pana, 743

Panaka, 417

Pana-karawu, 1031

Panduru, 1031

Panu-ala, 2352

Panu-habarala, 2361

Panu-kera, 773

Panu-kondal, 2250

Panu-nuga, 1973

Patak, 322

Patangi, 107

Pat-kala, 1S 11

Pat-kenda, 1947

Patola, 1;1

Patto-epala, 239

Patta-rralla, 1799

Pawatta, 1043
Peddimella, 1025

Pehimbiys, 378

Pelan, 1889

Pena-mihiriya, 171

Penda, 2390

Penela, 470, 71

Perela- $\pi$ el, 461

Pengiri-kurundu, 1769

Pengiri-mana, 2715

Peni-dodan, 57

Peai-tora, 673

Pepiliza, 1895

Pepol, 169

Pera, 152

Pera-tambala, 1347

Petan, 688

Petiks-wel, 33

Peti-tora, 675

Pichcha, 2.25

Pila, 553

Pilila, 1801-23

Pinibaru, 798

Pini-baru-tana, 2726

Pini-beraliya, 195

Pini-tuttiri, $27 \pm 8$

Pita-sudu-pala, 9\$1, 1685

Pitarrakka, 1864

Piyari, 417

Pol, 2331

Pol-kudu-pala, 1706

Polon-ala, 2353

Porums-mara, 1233

Poia-wel, 2367

Potu-bonchi, 100

Potu-honda, 883

Potu-kola, $25+5$

Potu-pala, 1405

Potu-pan, 2545

Pulun-imbul, 261

Pundalu, 477

Pupula, 1099, 1109

Puruk, 1580

Pus- $\pi$ el, 692

Puwak, 2311

Puwak-gediya-wel, 312

Rabu, 16

Radaliva, 503

Ramba, 2449

Rambuk, 2672

Rana-tampala, 301

Rana-wara, 676 
Ran-hirisa, 1137

Ran-kiriys, 2211

Ran-manisss, s5

Ran-motu, 2273

Ran-man-kikirindi. 1143

Rasa-kinda, 66

Rasa-mora, 474

Rasa-tel-kols, 141 ;

Rasni, 1173

Rata-8la, $3 \% 5$

Rata-attana, 262

Rata-bstu, 250

Rata-bilincha, 36

Rata-bulat-wel, 442. 1742

Rata-del, 31:5

Rata-endaru, 32i

Pata-ensal, 2230

Rata-corala, 29

Rata-gows. 144

Rata-hinguru, $28 \%$

Rata-jambu, 15.5

Raia-kaju, 88

Rata-kekiri, 173

Rata-kekuna, 63. 3:25

Rata-labu, 1 i 7

Ratambala, 1042

Rats-nelli, 321

Rata-pamba, 275

Rata-sapa, 3

Rata-tana, 2614

Rata-tira, 171

Rata-tora, 107. 11i

Rata-uguressa, 22

Rat-beraliys, 200

Rat-ekawerira. 1276

Rat-kelisa, its?

Rat-kihiri, 701

Rat-kohombs. 535

Rat-netul, $2: 21$

Rat-pitawakka, 1561, 1959

Rat-tans, 2657

Rattota, 1;1

Ratu-kimbulwenna, $17: 3$

Ratu-mihirisa, 173

Ratu-wa, 6\%?

Rawanidala. 956

Riti, 195:

Ruk, 1758

Ruk-8ttans, 1255

Samadara. 368

Sanninayan, 1096
Sop-sands, 1740

Sapu, 1

Sapu-mills, 1633

Sara, 958

Sembu-nerinchi, 314

Sendrikka, 296

Sepala, 228

Sepolika, 228

Sera, 2718, 350

Sewana-mediva, 1952

Sewandara, 2712

Sinuk, 1 \$1

Siri-bo, 1742

Siri-nelli, 324

Siyambala, 123

Sudu-idda, 1291

Sudu-kadumberisa, 1230

Sudu-kimbulwenns, 17:20

Sudu-liran, 1874

Sudu-niks, 1632

Sudu-pareri-mal, 206s

Sudu-puruk, 1595

Sudu-tampala, 1694

Sudu-tumba, 16:-

Sulu-nari, 1613

Suriya, 259

Surịa-mara, 706

Surandu-tals, 299

Suranda, 165

Tadala, 2360

Takkada, 116 s

Tal, 2330

Tals, 2319

Tambutu-फel, 2329

Tammanna, 1894

Tampala. 1704, 30?

Tanahal, 381

Tarana, $100 s$

Tamenna, 1763

Te, 3n

Tebu. 2209

Tela-kiriva, 1957

Telambu, 263

Tel-domba, 160

Tel-hirisa. 439

Teli-tana, 2725

Tel-kaduru, 1956

Tel-kekuna, 325

Tel-kola, $141 \mathrm{~s}$

Tel-tala. 15:2

Tembili. $\mathbf{2 3 3 1}$ 
Tembiliya, 785

Tibbatu, 1442

Tilo-guru, 126

Timbiri, 1221

Tiniya, 193

Tippili, 1741

Titta, 119

Titta-hondala, 884

Titta-kenda, 65

Titta-wel, 67

Titta-weralu, 299

Tolabo, 2240, 2242

Tolol, 119

Totila, 1518

To-wel, 442

Trastawalu, 1422

Tumba-karivila, 891

Tumpat-kurundu, 356

Tuttiri, 2704

Ubberiya, 742

Uguressa, 116

Ululu, 1770

Ulundu, 621

Una, 2804

Ura-genda, 145

Uru-hiri, 249]

Uru-honda, 401, 422

Uru-kanu, 401

Uru-tora, 674

Uru-wi, 2649

Uyala, 2245

Velala, 2346

Visnu-kranti, 1430

Wa, 678

Wada-kaha, 2370

Wadiya, 1010

Waga-pol, 356

Wal-aba, 88

Wal-amba, 484

Wal-asamodagan, 934

Wal-awara, 613

Wal-awari, 540

Wal-bevila, 232

Wal-bilin, 367

Wal-bombu, 1239

Wal-dambala, 630

Wal-diyalabu, 449

Wal-ehetu, 1981

Wal-ekaweriya, 996
Wal-enduru, 932

Wal-gan-miris-wol. 1747, 1748

Wal-gona, 1980

Wal-gonika, 1060

Wal-gurenda, 1635

Wal-handun, 1006

Wal-idda, 1291

Wal-inguru, 2228

Wal-jambu, 757

Wal-kaduru, 1278

Wal-kaha, 2200

Wal-kahambiliya, 1997

Wal-kapura-walliya, 1656

Wal-karapincha, 350

Wal-kekuna, 1917

Wal-kidaran, 2349, 2351

Wal-kinda, 64

Wal-kobbe, 464

Wal-kollu, 633, 634

Wal-kolondu, 1149

Wal-kopi, 1020

Wal-kudalu, 343

Wal-kurakkan, 2760

Wal-kurundu, 1766

Walla, 1766

Wal-lunu, 2243

Wal-me, 616

Wal-mediriya, 1233

Wal-meneri, 2609

Wal-mora, 468

Wal-munamal, 1965

Wal-murunga, 1886

Wal-nanu, 560

Wal-nawahandi, 916

Wal-niviti, 447

Wal-patpadagam, 985

Wal-pichcha, 1261

Wal-rasakinda, 909

Wal-rat-diyalabu, 454

Wal-ruk-attana, 229

Wal-sapu. 23

Wal-te-kola, 1146

Wal-tibbatu, 1444

Wal-trastawaln, 1428

Walu-kina, 162

Wal-waraka, 877

Wambatu, 254

Wampara, 21

Wana-mi, 1205

Wana-potu, 416

Wana-raja, 2161

Wana-sapu, 3 
Wanassa, 2816

Wanduru-me, 102

Wanduru-wel, 2328

Wan-epala, 1607

Wara, 1303

Wata-essa, 728

Wawiya, 1760

Weda-pana, 355

Wela, 89

Wel-a]a, 2360

Welanga, 271

Welangiriya, 102

Wel-aralu, 1969

Wel-beli, 1344

Wel-bendura, 2986

Wel-bute, 716

Wel-but-sarana, 1002

Wel-ehetu, 1966

Wel-embilla, 1186, 1800

Wel-hiri, 2409

Weli, 856

Weli-damba, 765

Weli-kaha, 856

Weli-penna, 744

Weli-piyana, 744

Weli-wenna, 1919

Wel-kahambiliya, 1932

Wel-kaparu, 1179

Wel-karal-heba, 1700

Wel-kayila, 1856

Wel-keliya, 283
Wel-keppitiya, 1909

Wellangiriya, 359

Wol-marukku, 2587

Wel-mediya, 283

Wel-mottu, 1333

Wel-radaliya, 504

Wel-ruk-attana, 1299

Weni-wel, 68

Weralu, 298

Weraniya, 964

Wesak-mal, 2072

Weta-kolu, 896

Wewarani, 1771

Wewel, 2322

Wila, 1475

Wire, 1890

Wisaduli, 1148

Yakada-wel, 33, 429

Yakahalu, 192

Yak-beriya, 529

Yak-erabadu, 609

Yak-eraminiya, 432

Yak-naran, 364

Yakkada-wel, 429

Yakkomadu, 897

Yak-wanassa, 1674

Yakutala, 2360

Yawakenda, 2205

Yogana-wel, 1085 
TAMIL.

Achehu, 286

Adampu, 803

Adatodai, 1607

Addula, 1376

Adutin-tappalai, 1739

Aglai, 391

Akatti, 87

Al, 1967

Allai, 2246

Amarai, 466

Ampallai, 501

Amulkkirai, 1446

Anaichovadi, 1102

Anaikuntumani, 693

Anaimulli, 699

Anai-nerinchi, 1521

Anaittadichchai, 458

Arachu, 341

Araikkirai, 1694, 1697

Ara-kiri, 2837

Arugam-pillu, 2756

Atalai, 1906

Atti, 689, 1986

Attuchankulai, 121

Attukaddupuli, 683

Attu-neddi, 565

Avarai, 676

Ayil, 1960

Chadachchi, 281

Chadavakku, 389

Chamai, 2610

Chandi, 298

Charanai, 920

Chatelai, 2030

Chattavarai, 2256

Chaturakalli, 1830

Chavandalai, 276

Chavukku, 347

Chaya, 988

Chelampai, 952

Chemmanathi, 308

Cheppunerinchi, 534

Chettupulu-kodi, 503

Chevakanpuclu, 232

Chidaventai, 44

Chilanti, 370

Chilanti-arichi, 2418

Chintil, 66

Chiruchemmanati, 309
Chiru-illantai, 394

Chiru-kandal, 737

Chiru-kila, 1275

Chiru-kirai, 1693

Chiru-nerinchi, 314

Chiru-paddi, 1378

Chiru-pilai, 1704

Chiru-piyari, 417

Chirup-padikkirai, 919

Chiruppayaru, 621

Chiru-pulichchul, 1376

Chitiviyarchenkalainir, 109:2

Chittakatti, 557

Chittamaddi, 231

Chittamanakku, 336

Chittirai-vempu, 378

Chittira-palavi, 1837

Chiva-charantai, 1124

Chivanarvempu, 535

Chomuntiri, 269

Chundan, 428

Churai, 431, 434, 172

Coco, 45

Devadaram, 308

Dimi-biya, 1385

Dommakottai, 160

Eddi, 1345

Elichchevi, 1713

Elilaippalai, 1285

Ella, 1522

Elumpurukki, 1780

Erapilakai, $345^{\circ}$

Errukalai, 1303

Erumaimullai, 1624

Ichanku, 1272

Ichavalai, 710

Ikkiri, 1572

Ilantai, 430

Iluppai, 1201

Inchu, 2318

Inji, 351 .

Irampai, 2449

Irumpalai, 1218

Itti, 1973

Iyamalai, 710

Iyanku, 1272

Iyavakai, 669

Juvarai, 1218 
Kachaddai, 804

Kachchalkodi, 1340

Kachchantirai, 922

Kadaikannai, 2609

Kadalıanchi, 395

Kadamanakku, 1633

Kaddamanakkı, 327

Kadduchchirakam, 1096

Kaddukodi, 73

Kaddu-ma, 484

Kaddumallikai, 1048

Kaddumuntiri, 447

Kaddunochehi, 1634

Kaddu-parutti, 260

Kaddupuli, 684

Kadukkai, 746

Kalkaipalai, 350

Kakkaipalai, 877, 1951

Kalatti, 1978

Kalli, 323

Kaloti, 391

Kaludai, 698

Kamukai, 2311

Kanchankorai, 1642

Kranchia, 1960

Kianchurai, 1345

Kandai, 346

Kandal, 735, 736

Kandankattari, 1443

Kanmuttankirai, 1040

Kanna, 1641

Kannakompu, 384

Kannu, 743

Kapila, 1943

Karai, 1011, 1037

Karanai, 1008

Karankutti, 1040

Karichcharanai, 1685

Karippalai, 1888

Karippan, 1141

Kari-vempu, 353

Karttikai-kilanku, 2270

Karukku-vaichchi, 418

Karumurukki, 116

Karunchurai, 99, 100

K̇arunkali, 1223

Karunochchi, 160I

Karuppu-thovori, 1230

Karuttappu, 626

Karuva, 1765

Karuvel, 696

Kíaru-veppal, 30
Kasitumpai, 1682

Katkarai, 371

Kattalai, 365

Kattikaya, 856

Kattimuruchan, 1265

Kattoddi, 94

Katukali, 116

Katukanni, 1220

Katu-peratti, 286

Kavali, 264

Kavani, 671

Kavarachu, 259

Kavilai, 553

Kavil-tumpai, 1386

Kaya, 785, 844

Kikkiri, 1572

Kilatti, 1275

Kilivai, 374

Kilkaynelli, 1864

Kilu-kiluppai, 522

Kinnai, 870

Kirai, 147

Kirimulla, 1572

Kodali-murunkai, 701

Koddi, 2379

Kodippayuru, 102

Kodi-taviddai, 277

Kolkottai, 156

Koli-avarai, 613

Kolinchi, 553

Koluk-kutti, 1625

Kona, 705

Kongu, 19

Konnai, 422

Korai, 2441

Korakkai-puli, 152

Kotanai, 1714

Kovvai, 889

Kula, 467

Kúla-pannai, 348

Kulappalai, 1302

Kuma, 468

Kumba, 2636

Tiumiddil, 1701

Kumil, 1630

Kuntu-mani, 598

Kuppa-meni, 1926

Kuppolay, 1099

Kurinclia, 1323

Kurinnan, 1311

Kurrokaya, 844

Kuruntu, 365 
Kuruvichehai, 1801-23

Kutiraivali, 574

Kuttukarasamatti, 542

Lechchaikedda, 298

Madalankai, 158

Makal, 120

Makil, 1213

Malai-parutti, 267

Malai-vempu, 380

Manakkovi, 1303

Manali, 927

Manaltakalli, 1435

Manchal, 350

Manchal-kadampa, 951

Manchavanna, 1050

Mangus, 28

Manipulnati, 1887

Mankalli, 1335

Mara-illipai, 36

Marai-tinni, 1939

Maral, 2237

Marungi, 761

Marutonti, 866

Marutu, 748

-Mayaladikkuruntu, 92, 366

Mayirmanikkam, 439

Metukku, 893

Milaku, 1744

Milla-kunari, 1908

Minni, 627

Mipullanti, 1856

Mochu-mochukkai, 899, 907

Montiri-kai, 7.5

Moongil, 2803

Motirikanni, 306

Muchuddai, 1390

Mudi-tumpai, 1681

Mudiya-kuntal, 1412

Mud-kirai, 1693

Mudkondai, 98

Mudpulanti, 1885

Mukalai, 1213

Mukavaliver, 550

Muk-karaichchi, 1685

Mulanninchil, 11 7, 943

Mulkarunkali, 1220

Mulkilivai, 375

Mullai, 1628

Mul-makil, 1199

Mulvenkai, 1840
Mullu-murnkku, 60

Mutirai, 392

Nagamulli, 1608

Naioringi, 1619 .

Naka, 169

Naka-kalli, 180

Naka-tali, 277

Nancharapanchan, 1320

Nannari, 1298

Nanti, 542

Narakaramba, 1108

Narankai, 57

Nari-ilantai, 431

Narippayaru, 619

Naruvili, 1371, 1372

Nattaichchuri, 1084

Naval, 780

Navala, 91

Navilankai, 91

Nayuruvi, 1709

Nedunurai, 37

Nelu, 1539

Nervalam, 329

Notavil, 1987

Neykkoddan, 470

Nilappanai, 2239

Nilavakai, 677

Nilavempu, 1584

Nir-kadampa, 952

Nir-mulli, 1532

Nir-naval, 766

Nir-nochchi, 1634

Nochchi, 1631

Nurai, 474

Nutai-pakal, 890

Odai, 697

Odi, 485

Oritad-tamari, 107

Pachumullai, 1627

Padri, 1520

Paduvan, 1533

Painkuray, 1040

Pakal, 890

Pakkilipal, 377

Pakkuvetti, 1377

Palai, 1214, 1328

Palam-padu, 227

Palam-pasi, 227

Palavi, 1836 
Palmadankai, 1290

Palmanikam, 1756

Palu-pakal, 891

Panai, 2330

Panalai, 470

Pandikaya, 844

Panichchai, 1221

Panir, 1024

Panittanki, 1432

Pannai, 354

Pappali, 169

Papparappuli, 41

Paraddai, 1534

Parasu, 612

Parutti, 248, 260

Pasalai, 1719

Pat-padakam, 924

Patpirai, 1993

Pavaddai, 1043

Pavaddai-kaya, 856

Pavettai, 1607

Perilantai, 431

Ptrukka, 41

Perumaddi, 250

Perumaruntu, 1740

Perum-kuruntu, 365

Peru-naval, 780

Peru-nerinchi, 1521

Perunkila, 1274

Perunkuruntu, 362

Perun-piyari, 418

Peruntutti, 235

Peykarumu, 2672

Puykkomaddi, 897

Pey-kuruntu, 364

Pey-maruddi, 1675

Poy-palai, 1320

Peypichukku, 896

Peyppatchotti, 1580

Peyppudal, 902

Peyt-tumpai, 1679

Pliandatullai, 605

Pichchuvilatti, 99

Pichukku, 895

Pichu-kodiya, 1701

Pikku, 895

Pila, 344

Pinari, 1635

Pinchul, 1635

Pirandai, 443

Pirasu, 1993

Piyari, 418
Podivilanga, 171

Podutalai, 1617

Ponnaimurankai, 706

Ponnankani, 1712

Ponnantakarai, 673

Pon-naru-vili, 1372

Potpattai, 1951

Priampu, 2322

Puchini, 175

Pudal, 886

Pudan, 1012

Pula, 1856

Puli, 123

Pulichchankirai, 142

Pulikkirai, 142

Pulinchakira, 36

Pullanti, 1856

Pumpulla, 195

Punaikkalaichchi, 665

Punairananki, 1926

Punaivirandi, 97

Punku, 654

Punnai, 160

Punnakalichi, 605

Punnikki, 274

Purankainari, 354

Puttalai, 1914

Puvarachu, 259

Puvu, 467

Raja-murunkai, 951

Ramsitha, 6

Ranai, 1771

Ruk-attana, 1285

Seemai-goraku, 29

Semel-panachai, 1237

Sitha, 5

Takarai, 674

Takkali, 250

Talai, 2332

Tali, 1400, 1417

Tamarai, 39

Tampanai, 1894

Tanti, 745

Tatta-payaru, 627

Taviddai, 283, 284

Tayirvalai, 89

$\mathrm{Te}, 30$

Tedkodukku, 1385

Tekil, 655 
Tennai, 2331

Tentukki, 1919

Teppaddi, 1909

Tetta, 1346

Tevataram, 308

Thavarai, 104

Thinakku, 755

Tilai, 1956

Tini, 147

Tippili, 1741

Tippilipana, 2315

Tirukkontai, 671

Tiruvatti, 688

Toggil, 1692

Topu-nelli, 1857

Tudari, 437

Tumpai, 891

Tumpalai, 206

Tutuvalai, 1444

Tuvadi, 437

Udai, 697

Udai-el, 698

Uluntu, 621

Uluvintai, 39

Umiri, 1718

Urkkovi, 1303

Uttamalkam, 1305

Uvay, 1271

Uyil, 708

Vaddatutti, 235, 236, 251

Vaddu, 1443

Vaddu-takarai, 675

Vakai, 678, 705

Vakkai, 672

Vakkana, 1220

Vallampuri, 270

Vallarai, 930

Vammi, 949

Vandakkay, 37

Vankiruvalai, 917

Vanni, 109

Varittulai, 1951

Vatamadakki, 1636, 1637

Vatchikuran, 1031

Vattakanni, 1946
Vedehi, 1042

Vedukkanari, 1219

Vekkali, 749

Velayil, 1960

Veliparatti, 1305

Vella, 1021

Vellai-kadampa, 950

Vellai-karunkali, 1228

Vellai-thoveri, 1222

Vellaruku, 1362

Veluruvai, 651

Velvel, 700

Vempadam, 429

Vempu, 381

Venkai, 653

Venkalikaya, 785, 856

Vennachchi, 94

Vennochehi, 632

Ventonti, 2270

- Venumattai, 1447

Vetpavaddai, 1046

Vettilai, 1742

Vette-ver, 2712

Vichamunkil, 2240, 2242

Vichnu-kiranti, 1430

Vidattal, 695

Vidi, 1371

Vid-pani, 275

Vila, 366

Vilatti, 366

Vili, 93

Vilpadri, 1519

Vilvam, 60

Vilva-padri, 1213

Vinanku, 271

Virai, 1890

Virali, 478

Visa, 1847

Vitchurunai, 1888

Vitlikanna, 1197

Vivay, 1271

Waragu, 2569

Yar, 1084

Yavaranai, 1771

Yerkoli, 1031 


\section{ENGLISH NAMES.}

African rubber, 230

Alligator pear, 319

Almond, country, 145

Almond, Java, 63

Aloe, American, 358

American aloe, $35 \mathrm{~S}$

Annatto, 20

Apple, elephant, 366

Apple, love, 250

Apple, thorn, 261

Arabian coffee, 193

Arabian jasmine, 225

Arecanut palm, 2311

Arnatto, 20

Arrowroot, 354

Artichoke, 215

Artichoke, Jerusalem, 20.5

Arum lily, 374

Ash pumpkin, 175

Assam indiarubber, 342

Avocado, 319

Australian blackwood, 132

Bael fruit, 60

Balsam, 322

Bamboo, 2804

Bamboo, Chinese, 2805

Bamboo, dwarf, 2805

Bamboo, giant, 385

Bamboo, spiny, 2803

Banana, 2235

Bandakai, 37

Banyan, 1967

Baobab, 41

Barberry, 77

Bark, red, crown, \&c., 189-91

Basil, sweet, 289

Bastard obony, 1227

Batticaloa orchid, 2122

Bead treo, 64

Bean, 99-102

Bean, sacred, 80

Beetroot, 307

Pengal kino, 612

Bermuda grass, 2756

Betel-nut palm, 2311

Betel pepper, 1742

Bird pepper, 258

Blackborry, 716
Black gram, 621

Black wattle, 130

Black wood, 105

Black wood, Australian, 13:?

Blimbing, 53

Blue gum, 147

Bottle gourd, 172

Boxwood, Ceylon, 1031

Brazil cherry, 155

Breadfruit, 345

Brinjal, 254

Brown bark, 191

Bullock's heart, 6

Bulrush millet, 2636

Buttercup, 6

Cabbage, 14

Cabbage rose, 140

Cacao, 45

Caffre $\lim \theta, 56$

Calabash encumber, 17:

Calamander, 1229

Calumba, false, 68

Camphor, 318

Candle nut, 325

Cape gooseberry, 257

Cape pondweed, 376

Cardamom, 2230

Carrot, 186

Cashew, 75

Cassava, 331

Castilloa rubber, 343

Castor oil, 3.36

Ceara rubber, 3.32

Colery, 181

Century plant, 358

Ceylon boxwood, 1031

Ceylon oak, 467

Champak, 1

Chareoal tree, 196:3

Chayote, 179

Chay-root, $98 \mathrm{~s}$

Cherimoyer, $\delta$

Cherry boan, 102

Chicken poa, 92

Chickweed, 23

Chilly, 259

Chinese bamboo, 280;

Chittagong wood, 391 
Cliocho, 179

Chocolate, 45

Cholam, 379

Cinnamon, 1765

Cinnamon, wild, 1790

Citronella grass, 2715

Citronella grass, Winter's, 2716

Clearing nut, 1346

Clove, 156

Clover, Dutch, white, 82

Coca, 48

Cochin goraka, 29

Cock's foot, 384

Cockspur thorn, 698

Cocoa, 45

Coconut, 2331

Coffe日, 193, 194

Coffee, wild, 1020

Colocynth, 897

Coriander, 185

Cotton, 39

Cotton, Sea island, 40

Cotton tre $\theta, 260$

Country almond, 145

Country potato, 291

Cowitch, 607

Crab's eyes, 598

Croton, 330

Croton oil plant, 329

Crown bark, 189, 191

Cucumber, 173

Curry bean, 99

Ciurry leaf, 353

Custard apple, 7

Cypress, Monterey, 387

Dadap, 96

Daffodil orchid, 2101

Dandelion, 216

Devil nettle, 1999

Dhal, 104

Divi-divi, 109

Doob grass. 2756

Doon, red, 190, 191

Dracæna, 366

Dum palm, 371

Durian, 42

Dutch clover, 82

Dwarf bambno, 2805

East Indian rosewood, 105 Ebony, 1223
Egg plant, 254

Egyptian lotus, 78

Elephant apple. 366

Hephant's ears, 1778

Eleplant thom, 699

Élm, Inclian, 1960

Eve's apple, 1284

False calumba, 68

False jalap, 296

Fever nettle, 1999

Flamboyante, 110

Forbidden fruit, 1284

Forget-me-not, 1388

Fox-tail orchid, 2122

French bean, 100

Furniture leaf, 1982

Furze, 80

Gall-nut, 746

Gamboge, 153

Ganja, 338

Garlic. 369

Giant bamboo, 38.5

Gingelly, 1522

Ginger, 351

Gingili, 1522

Globe amaranth, 303

Goat weed, 195

Gold molhur, 110

Gooseberry tomato, 257

Goraka, Cochin, 2.9

Gorse, 80

Gourd, 171, 172, 176

Gram, black, green, 621

Gram; horse, Madras, 10:3

Granadilla, 164

Grape, 70

Grass, Bermuda, 2756

Grass, citronella, 2715

Grass, citronella, Winter's, 2716

Grass, doob, 2756

Grass, Guinea, 2614

Grass, lemon, 2718

Grass, Mauritius, 2593

Grass, water, 2593

Green gran, 621

Groundnut, 88

Guango, 136 
Guava, 152

Guava, wild, 756

Guinea com, 379

Guinea grass, 2614

Gum, blue, 147

Gunpowder plant, 346

Harebell, 1174

Har'e's foot trefoil, $\delta 4$

Hemp, 524, 338

Henna, 866

Hog plum, 501

Hollyhock, 31

Horse cassia, 113

Horse gram, 10.3

Horseradish, 12

Horseradish tree, 77

Hyacinth orclid, 21:17

Indian cork tree, $27 t$

Indian corn, $37 \%$

Indian elm, 1960)

Indian lilac, 64

Indian liquorice, 598

Indian mahogany, 68

Indian nulberry, 340

Indian $\operatorname{ros} \theta, 141$

Indian sliot, 2234

Indigo, 544, 84

Ink-nut, 746

Ipecacuanha, wild, $132(1,210$

Iron bark, 149

Ironwood, 169

Italian millet, 381

Jak, 344

Jalap, false, 296

Jarrall, 151

Jasinine, Arabian, 225

Java almond, 63

Jequié rubber, 333

Jernisalem artichokt, 205

Job's toars, 2661

Judas's bag, 41

Jungle nail, 699

Jute, 292, 293

Khas-khas, 2712

Kidney beall, 100

Kola, 13
Kollu, 103

Kurakkan, 383

Lantana, 283

Le日che日, 72

Le日k, 368

Lemon grass, 2718, 380

Lettuce, 217

Lettuce tree, 298

Liberian $\operatorname{coffe\theta }, 194$

Lilac, Indian, Persian, 64

Lily of the valley orchid, 2093

Lima bean, 99

Lime, 59

Lime, Caffre, 56

Litchi, 72

Longan, 474

Lotus, 80

Lotus, Egyptian, 78

Love apple, 250

Love grass, 2704

Love-lies-bleeding, 299

Lovi-lovi, 21

Macassar kernels, 61

Macassar oil, 3

Nace, 377

Madagasear poriwinkle, 231

Madras gram, 103

Madias thorn, 135

Madre de cacao, 97

Mallogany, $66^{\circ}$

Mahogany, large leaved, 67

Mahogany, swamp, 1j0

Maize, 377

Malabar nut, 1607

Maley apple, 153

Mango, 74

Mangoste日n, 28

Mangrov $\theta, 735,736,739$

Mangrove, white, 1641

Manikwatte weed, 51

Manioca, 331

Margosa, 381

Marigold, 211, 21:

Marvel of Peru, 296i

Matara tea, 676

Mauritius grass, 2593

Mauritius liemp, 359

Mignonette, wild, $19 . \mathrm{s}$ 
Milk hedge, 323

Millet, 379

Millet, Italian, 381

Mint, 293

Monkey-nut, 88

Monterey Cypress, 387

Moon-flower, 1402

Mountain papaw, 170

Mugwort, 1149

Mulberry, Indian, 340

Mulberry, paper, 339

Mullein, 267

Myrobalans, 745

Nam-nam, 124

Nasturtium, 50

Nettle, devil, fever, 1999

Nettle, Nilgiri, 2000

New Zealand flax, 364

Nilgiri nettle, 2000

Nutmeg, 317

Nux-vomica, 1345

Oak, Ceylon, 467

Oak, patana, 804

Oil palm, 372

Okra, 37

Oleander, 237

Olive, wild, 298

Onion, 367

Orange, 57

Orchid, Batticaloa, 2122

Orehid, daffodil, 2101

Orchid, fox-tail, 2122

Orchid, hyacinth, 2197

Orchid, lily of the valley, 2093

Orchid, pigeon, 2184

Orchid, primrose, 2073

Orchid, white dove, 2068

Puddy, wild, 2649

Palm, arecanut, 2311

Palm, coconut, 2331

Palm, dum, 371

Palm, oil, 372

Palm, palmyra, 2330

Palm, royal, 370

Palm, toddy, 2315

Palmyra, 2330
Palta, 319

Papaw, 169

Papaw, mountain, 170

Paper mulberry, 339

Para rubber, 325

Parsley, 183

Parsnip, 184

Passion fruit, 166

Patana oak, 804

Pea, 93

Peach, 137

Peacock flowor, 108

Pea nut, 88

Pear, 142

Pearlwort, 24

Pepper, betel, 1742

Pepper, black, 1744

Pepper, long, 1741

Pepper, red, 259

Periwinkle, 232

Periwinkle, Madagascar, 231

Persian lilac, 64

Physic nut, 327

Pigeon orchid, 2184

Pigeon pea, 104

Pimpernel, 222

Pineapple, 355

Pitcher-plant, 1737

Plantain, 295

Plantain, wild, 2235

Phum, hog, 501

Plum, sapodilla, 224

Poinsettia, 321

Pomegranate, 158

Pomelo, 58

Poor man's weather glass, 222

Potato tree, 253

Primrose orehid, 2073

Prince of Wales's feathors, 300

Pumpkin, 178

Radish, 16

Ragi, 383

Rain tree, 136

Rambong, 342

Rambutan, 73

Red bark, 190

Red cedlar, 68

Red doon, 190, 191

Red pepper, 259

Red toon, 69 
Renanso rubber, 334

Rhea, wild, 2020

Rice, 2649

Rice paper tree, 188

Rose, 140,141

Rose apple, 154

Roucou, 20

Royal palm, 370

Rozelle, 36

Rubber, African, 230

Rubber, Assam, 342

Rubber, Castilloa, 343

Rubber, Ceara, 332

Rubber, Jequié, 333

Rubber, Para, 325

Rubber, Remanso, 334

Sabre bean, 613

Sacred bean, 80

Sapodilla plum, 224

Sappan, 107

Sapu, 1

Satinwood, 392

Scrow-pine, 2332

Sebestens, 1371

Sensitive plant, 128

Shaddock, 58

Sliepherd's purse, 15

Shoe flower, 38

Shola-pith, 565

Silk-cotton tree, 261

Silky oak, 320

Silver wattle, 131

Snake gourd, 171

Soursop, 5

Sow-thistle, 218, 219

Spanish needle, 1146

Speedwell, 272

Spiny. bamboo, 2803

Spurrey, 25

Strawberry, 139

Strawberry tomato, 257

Sugar apple, 7

Sugar cane, 378

Sundew, 728, 729, 730

Sunflower, 204

Sunflower, wild, 203

Sunn hemp, 524

Swamp mahogany, 150

Sweet basil, 289

Swent cup, 166
Swoet flag, 2370

Sweet potato, 243

Sweet sop, 7

Sweet vernal grass, 38\%

Talipot, 2319

Tallow tree, 337

Tamarind, 123

Tamarind, velvet, 684

Tamarisk, 147

Tapioca, 331

Taro, 2360

Tea, 30

Teak, 287

Tea, Matara, 676

Tea rose, 141

Telegraph plant, 596

Temple tree, 233

Thorn apple, 261

Tigers' claws, 277

Toddy palm, 2315

Tomato, 250

Tomato, strawberry, gooseberry, 257

Tomato, tree, 255

Toothache plant, 1145

Tree mignonette, 866

Tree tomato, 255

Trincomalee wood, 276

Trumpet flower, 262

Tulip tree, 259

Turmeric, 350

Umbrella tree, 697

Upas tree, 1987

Vanilla, 348

Vegetable narrow, 178

Velvet tamarind, 684

Violet, 104, 105, 106, 18

Watercress, 8

Water grass, 2593

Water lemon, 168

Water lettuce, 2338

Wrater lily, 78, 79

Water molon, 174

Wattle, 130, 131 


\section{( 188$)$}

Whin, 80

White clover, 82

White dove orchicl, 2068

White weed, 195

Wild cinnamon, 1790

Wild coffer, 1020

Wild guava, 756

Wild ipecacuanla, 1320,240

Wild olive, 298

Wild paddy, 2649
Wild plantain, 2235

Wild sunflower, 203

Winter's citronella grass, 2716

Wood apple, 366

Worm seed, 305

Yellow bark, 189

Ylang-ylang, 3

H. C. COTTLE, GOVERNMENT PRINTER, COLOMBO, CEYLON. 







$$
\text { . }
$$

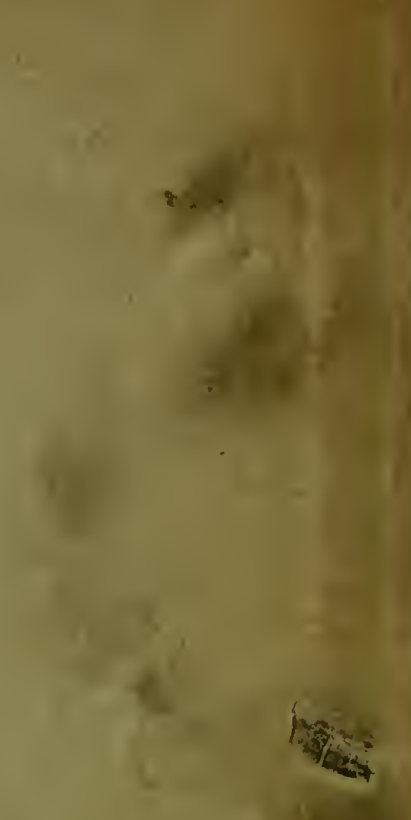

